Supplement of Atmos. Chem. Phys. Discuss., 15, 23651-23681, 2015

http://www.atmos-chem-phys-discuss.net/15/23651/2015/

doi:10.5194/acpd-15-23651-2015-supplement

(C) Author(s) 2015. CC Attribution 3.0 License.

(c) (i)

\title{
Pesticides in the atmosphere: a comparison of gas-particle partitioning and particle size distribution of legacy and current-use pesticides
}

\section{Degrendele et al.}

Correspondence to: J. Klánová (klanova@ recetox.muni.cz)

and L. Melymuk (melymuk@ recetox.muni.cz)

The copyright of individual parts of the supplement might differ from the CC-BY 3.0 licence. 
Table of Contents:

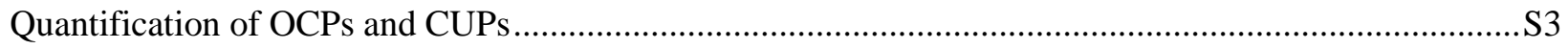

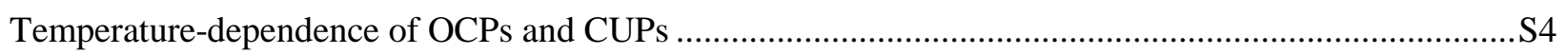

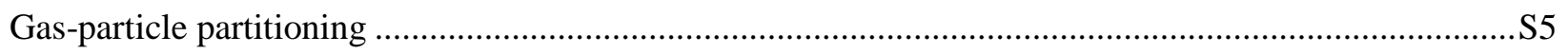

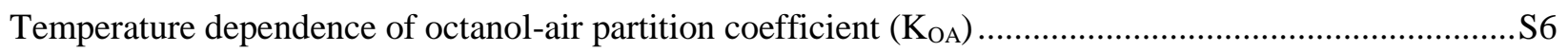

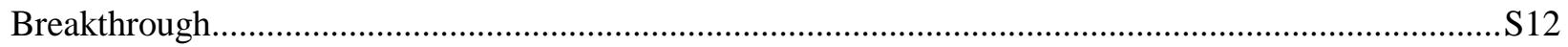

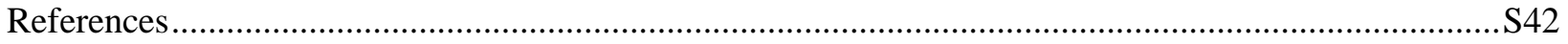

\section{List of Tables}

Table S1: Air sample volumes collected for OCP analysis from background site. ..................................S7

Table S2: Air sample volumes collected for CUPs analysis from background site................................S8

Table S3: Cascade impactor air sample volumes collected for OCPs analysis. ......................................S9

Table S4: Cascade impactor air sample volumes collected for CUPs analysis. .....................................S10

Table S5: Selected LC-MS/MS experimental parameters, limits of detection and limits of quantification

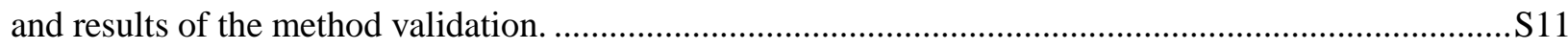

Table S6: Results of breakthrough experiments for CUPs. ................................................................. 12

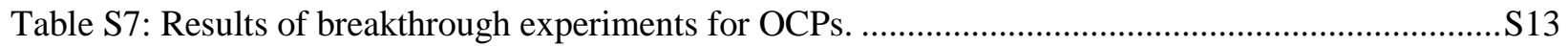

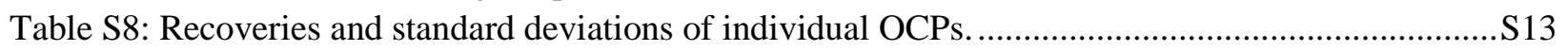

Table S9: Recoveries and standard deviations of individual CUPs.......................................................

Table S10: Use of agricultural pesticides in the Czech Republic in 2012 ............................................S15

Table S11: Use of agricultural pesticides in the Czech Republic in 2013 ...........................................S16

Table S12: Concentration of individual CUPs in the gaseous phase at the background site...................S17

Table S13: Concentration of individual CUPs in the particulate phase at the background site..............S21

Table S14: Concentrations of individual OCPs in the particulate phase .............................................S25

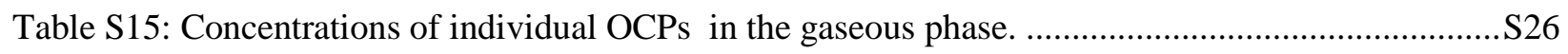

Table S16: Results of Clausius-Clapeyron plots showing slopes, constants and coefficient of determination for OCPs..

Table S17: Results of Clausius-Clapeyron plots showing slopes, constants and coefficient of

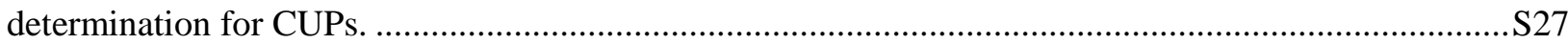

Table S18: Octanol-air partitioning coefficients, $\mathrm{K}_{\mathrm{OA}}$, of OCPs and CUPs at $25^{\circ} \mathrm{C}, \mathrm{T}_{\min }$ and $\mathrm{T}_{\max } \ldots \ldots . . . \mathrm{S} 28$ Table S19: Particulate concentrations of individual OCPs according to particle size at the rural and the

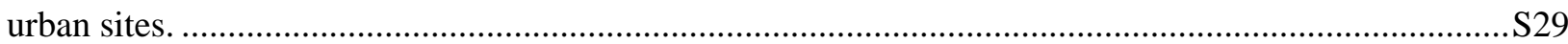

Table S20: Particulate concentrations of individual CUPs according to particle size at the rural and urban

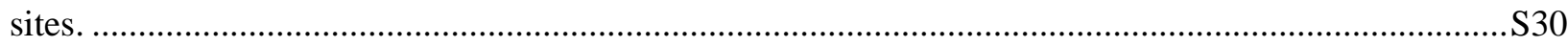

Table S21: Particle-phase concentrations of individual OCPs according to particle size at the rural and the urban sites. . S34

Table S22: Particulate concentrations of individual CUPs according to particle size at the rural and urban sites. .S36 


\section{List of Figures}

Figure S1: Map of the sampling sites....

Figure S2: Monthly concentrations of terbuthylazine, $p, p^{\prime}$-DDT and fenpropimorph.

Figure S3: Linear regression of $\log \mathrm{K}_{\mathrm{OA}}(\mathrm{T})$ versus $\log \mathrm{K}_{\mathrm{OA}}\left(\mathrm{T}_{\text {ref }}\right)$ of $\mathrm{OCPs}, \mathrm{CBs}, \mathrm{PCBs}, \mathrm{PAHs}, \mathrm{CAR}$ and PCNs for

$\mathrm{T}_{\min }$ and $\mathrm{T}_{\max }$ used to determine the log $\mathrm{K}_{\mathrm{OA}}$ of all CUPs except chlorpyrifos.

Figure S4: Comparison of logK $\mathrm{OA}_{\mathrm{OA}}$ values of chlorpyrifos at different temperatures determined based on

experimental values and extrapolated from the regression analysis. 


\section{Quantification of OCPs and CUPs}

OCPs:

OCPs were analyzed and quantified by gas chromatography coupled to a tandem mass spectrometer (GCMS/MS). Samples were analyzed with three slightly different sets of parameters/instrument configurations due to changes in laboratory instrumentation during the dates of sample collection and processing.

High volume air samples (PUF and filter) collected from the January to June 2012 were analyzed on an Agilent 6890N GC coupled to Waters Quattro Micro GC. The column used for chromatographic separation was $60 \mathrm{~m}$ x $0.25 \mathrm{~mm} \times 0.25 \mu \mathrm{m}$ DB5-MSUI (Agilent, J\&W). Injection volume was $1 \mu \mathrm{L}$. The temperature program was: $80^{\circ} \mathrm{C}, 15^{\circ} \mathrm{C} / \mathrm{min}$ to $180^{\circ} \mathrm{C}, 5^{\circ} \mathrm{C} / \mathrm{min}$ to $300^{\circ} \mathrm{C}$. The inlet temperature was $280^{\circ} \mathrm{C}$. The carrier gas was He with a flow rate of $1.5 \mathrm{~mL} / \mathrm{min}$. High volume air samples (PUF and filter) collected from the June 2012 to January 2014 were analyzed on an Agilent 7000B GC/MS Triple Quadrupole, with SGE HT$8(60 \mathrm{~m} \times 0.25 \mathrm{~mm} \times 0.25 \mu \mathrm{m})$ column. The injection volume was $3 \mu \mathrm{L}$. The temperature program was: $80^{\circ} \mathrm{C}(1.5 \mathrm{~min}), 40^{\circ} \mathrm{C} / \mathrm{min}$ to $200^{\circ} \mathrm{C}(18 \mathrm{~min}), 5^{\circ} \mathrm{C} / \mathrm{min}$ to $305^{\circ} \mathrm{C}(21 \mathrm{~min})$. The inlet temperature was $280^{\circ} \mathrm{C}$. The carrier gas was He with a flow rate of $1.5 \mathrm{~mL} / \mathrm{min}$.

Cascade impactor samples were analyzed for OCPs by GC-MS/MS (Agilent 6890N coupled to Waters Quattro Micro GC) with a J\&W Scientific DB-5-MS fused silica column (60 m x $0.25 \mathrm{~mm}$ x $0.25 \mu \mathrm{m}$ ). The injection volume was $1 \mu \mathrm{L}$. The temperature program was: $80^{\circ} \mathrm{C}(1 \mathrm{~min}), 15^{\circ} \mathrm{C} / \mathrm{min}$ to $180^{\circ} \mathrm{C}, 5^{\circ} \mathrm{C} / \mathrm{min}$ to $300^{\circ} \mathrm{C}$. Injection was splitless at $280^{\circ} \mathrm{C}$, interface held at $280^{\circ} \mathrm{C}, \mathrm{He}$ constant flow $1.5 \mathrm{~mL} / \mathrm{min}$.

LOQs of samples collected from January to December 2012 were determined from calibration curves and were $1 \mathrm{ng} / \mathrm{mL}$ for each compound. This corresponds to approximately $0.22 \mathrm{pg} . \mathrm{m}^{-3}$ with our active sampling configuration. LOQs of samples collected from January to December 2013 were determined for each compound and for each batch of samples by the quantification software (TargetLynx®) and were defined as a peak with the signal/noise ratio of 9 in the respective chromatograms of the lowest point of the calibration curve $(1 \mathrm{ng} / \mathrm{mL})$. For those samples, LOQs ranged from 37.7 to $365 \mathrm{pg} \cdot \mathrm{mL}^{-1}$ which corresponds to about 0.008 to $0.08 \mathrm{pg} . \mathrm{m}^{-3}$ with our sampling configuration. Finally, for cascade impactor samples, LOQs were also determined by TargetLynx ${ }^{\circledR}$ for each compound and each sample in the sample chromatograms. LOQs of individual samples ranged from 0.014 to $15 \mathrm{ng} . \mathrm{mL}^{-1}$ which corresponds to 0.0005 to 0.54 pg. $\mathrm{m}^{-3}$ for our active sampler configuration. 
CUPs:

Separation of CUPs was performed by LC-MS using an Agilent 1290 HPLC (Agilent Technologies, Waldbronn, Germany) consisting of a vacuum degasser, a binary pump, a thermostated autosampler $\left(10^{\circ} \mathrm{C}\right)$, and a thermostated column compartment kept at $30^{\circ} \mathrm{C}$. The column was a Phenomenex Synergi Fusion C18 endcapped $(3 \mu \mathrm{m}) 100$ x $2.1 \mathrm{~mm}$ i.d., equipped with a Phenomenex SecureGuard C18 guard column (Phenomenex, Torrance, CA, USA). The mobile phase consisted of $5 \mathrm{mM}$ ammonium acetate in water (A) and $5 \mathrm{mM}$ ammonium acetate in methanol (B). The binary pump gradient was non-linear (increase from $20 \% \mathrm{~B}$ at $0 \mathrm{~min}$ to $80 \% \mathrm{~B}$ at $1 \mathrm{~min}$, then increase to $90 \% \mathrm{~B}$ at $5 \mathrm{~min}$, then $90 \% \mathrm{~B}$ for $8 \mathrm{~min}$ and $5 \mathrm{~min}$ column equilibration at initial conditions $(20 \% \mathrm{~B})$ ); the flow rate was $0.25 \mathrm{~mL} / \mathrm{min}$. $10 \mu \mathrm{L}$ of individual sample was injected for the analyses. CUPs were quantified using a mass spectrometer (AB Sciex Qtrap 5500, AB Sciex, Concord, ON, Canada) with electrospray ionization (ESI). Ions were detected in the positive mode. The ionization parameters were as follows: capillary voltage, $5.5 \mathrm{kV}$; desolvation temperature, $400^{\circ} \mathrm{C}$; Curtain gas 15 psi, Gas1 40 psi, Gas2 30 psi. The m/z transitions presented in Table S5 were monitored in scheduled MRM mode (with corresponding values of declustering potential - DP $(\mathrm{V})$, entrance potential - EP (V), collision cell exit potential - CXP(V) and collision energy - CE (V)). Values of method quantification limits (MQL) and retention times are also presented in Table S5. LODs and LOQs were estimated as the quantity of analyte with a signal to noise ratio of 3:1 and 10:1, respectively. The method was validated by quantifying spiked pre-cleaned PUFs and QFFs, for which the recoveries of individual CUPs ranged from $52.4 \% \pm 21.4$ for disulfoton to $115 \% \pm 17.4$ for alachlor (Table S9).

\section{Temperature-dependence of OCPs and CUPs}

The gas-phase behavior of SVOCs can be described by the Clausius-Clapeyron equation:

$\operatorname{Ln} P=\left(-\Delta H_{v} / R\right)(1 / T)+$ constant

where $\mathrm{P}$ is partial pressure $(\mathrm{Pa}), \mathrm{T}$ is temperature $(\mathrm{K}), \Delta \mathrm{H}_{\mathrm{v}}$ is enthalpy of vaporization $\left(\mathrm{kJ} \cdot \mathrm{mol}^{-1}\right)$ and $\mathrm{R}$ is the gas constant. The temperature dependence of atmospheric pesticide concentrations was expressed as the linear regressions of the natural logarithm of partial pressure versus the inverse of the temperature:

$\operatorname{Ln} \mathrm{P}=\mathrm{m} / \mathrm{T}+\mathrm{b}$, where $\mathrm{m}$ and $\mathrm{b}$ are constants.

(Eq. S2)

Partial pressures of individual compounds were calculated for each sampling event using gas phase concentrations and the ideal gas law. 


\section{Gas-particle partitioning}

Partitioning of organic compounds such as pesticides between the gas and particle phases is often described

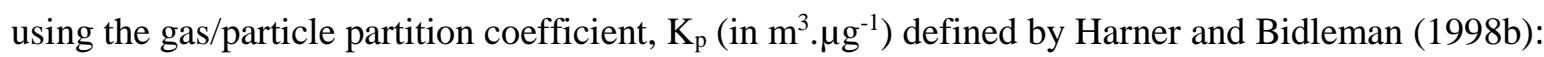

$$
\mathrm{K}_{\mathrm{p}}=\left(\mathrm{C}_{\mathrm{p}} / \mathrm{C}_{\mathrm{TSP}}\right) / \mathrm{C}_{\mathrm{g}}
$$

where $\mathrm{C}_{\mathrm{p}}$ and $\mathrm{C}_{\mathrm{g}}$ are the concentrations of the pesticides (in pg. $\mathrm{m}^{-3}$ ) in the particulate and gaseous phases, respectively and $\mathrm{C}_{\text {TSP }}$ is the concentration of the total suspended particles (TSP) in the air (in $\mu \mathrm{g} \cdot \mathrm{m}^{-3}$ ).

$\mathrm{K}_{\mathrm{p}}$ can be predicted using the octanol-air partition coefficient $\left(\mathrm{K}_{\mathrm{OA}}\right)$. This assumes that absorption into particulate organic matter of the particles determines the distribution process, while other types of molecular interaction are neglected (Harner and Bidleman, 1998b).

Then, $\mathrm{K}_{\mathrm{p}}$ can be defined as:

$\mathrm{K}_{\mathrm{p}}=\frac{\mathrm{f}_{\mathrm{OM}} \mathrm{MW}_{\mathrm{OCT}} \zeta_{\mathrm{OCT}}}{\rho_{\mathrm{OCT}} \mathrm{MW}_{\mathrm{OM}} \zeta_{\mathrm{OM}}{ }^{12}} \mathrm{~K}_{\mathrm{OA}}$

where $f_{O M}$ is the fraction of organic matter $(\mathrm{OM})$ phase on particles, $\mathrm{MW}_{\mathrm{OCT}}$ and $\mathrm{MW}_{\mathrm{OM}}$ are the mean molecular weights (in g. $\left.\mathrm{mol}^{-1}\right)$ of octanol and OM, respectively,

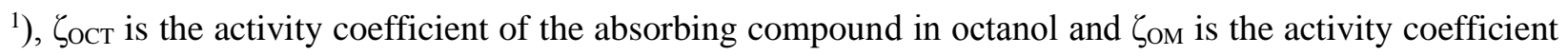

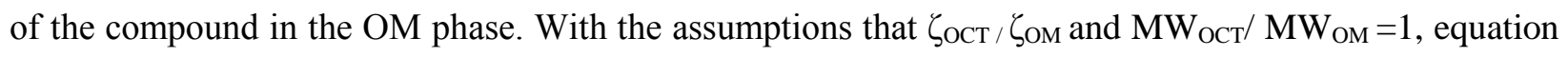
S4 can be written as:

$\log \mathrm{K}_{\mathrm{p}}=\log \mathrm{K}_{\mathrm{OA}}+\log \mathrm{OM}_{\mathrm{M}}-11.91$

Finally, predicted particulate fractions $\left(\theta_{\mathrm{pr}}\right)$ can be calculated using:

$\theta_{\mathrm{pr}}=\mathrm{K}_{\mathrm{p}} \mathrm{C}_{\mathrm{TSP}} /\left(1+\mathrm{K}_{\mathrm{p}} \mathrm{C}_{\mathrm{TSP}}\right)$

In this study, a fom of 0.2 was used and determined to be reasonable for this background site, based on a research on particles at the European level (Putaud et al., 2004). As TSP values were not available at the sampling location, the hourly $\mathrm{PM}_{10}$ concentrations at this site from 2012 were used instead (data provided by the Czech Hydrometeorological Institute, http://www.chmi.cz)

It should be noted that the $\mathrm{K}_{\mathrm{OA}}$-based model might not give the best results for the more polar CUPs (dimethoate, metamitron, pyrazon and carbendazim) (Götz et al., 2007; Sadiki and Poissant, 2008) because

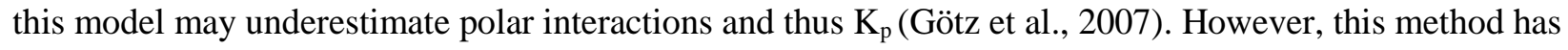
previously produced reasonable results for CUPs (Sauret et al., 2008; Yao et al., 2008). Moreover, given 
the limited available data for CUPs and the desire for comparability between OCPs and CUPs within this dataset, determination of $\mathrm{K}_{\mathrm{p}}$ using $\mathrm{K}_{\mathrm{OA}}$ was the best choice for the current study.

\section{Temperature dependence of octanol-air partition coefficient $\left(\mathrm{K}_{\mathrm{OA}}\right)$}

Due to the large enthalpy change involved in octanol to air transfer, a strong temperature dependence of the octanol-air partition coefficient ( $\mathrm{K}_{\mathrm{OA}}$ ) is expected (Harner and Mackay, 1995). When feasible, temperature dependence of $\mathrm{K}_{\mathrm{OA}}$ of the studied pesticides was determined from published relationships, based on direct measurements (Odabasi and Cetin, 2012a; Shoeib and Harner, 2002a; Zhang et al., 2009). However for most CUPs, no information on the temperature dependence of $\mathrm{K}_{\mathrm{OA}}$ was available. In lieu of this, temperature adjusted $\mathrm{K}_{\mathrm{OA}}$ values were determined from an extrapolation of available experimental values determined for organochlorine pesticides (OCPs) (Odabasi and Cetin, 2012b; Shoeib and Harner, 2002b; Zhang et al., 2009), chlorobenzenes (CBs) (Harner and Mackay, 1995), polychlorinated biphenyls (PCBs) (Harner and Mackay, 1995), polycyclic aromatic hydrocarbons (PAHs) (Odabasi et al., 2006a), carbazole (CAR) (Odabasi et al., 2006b) and polychlorinated naphthalenes (PCNs) (Harner and Bidleman, 1998a). Except for prochloraz and temephos ( $\log \mathrm{K}_{\mathrm{OA}}=13.6$ and 13.1, respectively), the range of $\log \mathrm{K}_{\mathrm{OA}}$ of CUPs (7.49$11.2)$ is a sub-range of the range of $\log \mathrm{K}_{\mathrm{OA}}$ (4.36-12.6) spanned by these classes of compounds. To this end, the $\log \mathrm{K}_{\mathrm{OA}}$ values of OCPs, CBs, PCBs, PAHs, CAR and PCNs for each sampling temperature were plotted versus their corresponding values at the reference temperature $\left(25^{\circ} \mathrm{C}\right)$. Examples of these plots are provided in Figure S3. Finally, using the linear regression relationship from these plots $\left(r^{2}>0.992\right), \log K_{O A}$ values of CUPs at the specific temperatures were calculated. $\log \mathrm{K}_{\mathrm{OA}}$ values of OCPs and CUPs at $25^{\circ} \mathrm{C}$, $\mathrm{T}_{\text {min }}$ (winter) and $\mathrm{T}_{\max }$ (summer) are shown in Table S15. We tested this method for chlorpyrifos and the

differences in $\log \mathrm{K}_{\mathrm{OA}}$ values at different temperatures determined based on the experimental values and those extrapolated from the regression analysis were negligible (Table S15). 
Table S1: Air sample volumes collected for OCP analysis from background site. Sample volumes were corrected for temperature and pressure.

\begin{tabular}{|c|c|c|}
\hline Sampling start & Sampling end & Sampling volume $\left(\mathrm{m}^{3}\right)$ \\
\hline $11 / 01 / 2012$ & $18 / 01 / 2012$ & 4559.3 \\
\hline $25 / 01 / 2012$ & $01 / 02 / 2012$ & 4576.5 \\
\hline 08/02/2012 & $15 / 02 / 2012$ & 8103.3 \\
\hline $22 / 02 / 2012$ & $29 / 02 / 2012$ & 4532.5 \\
\hline 07/03/2012 & $14 / 03 / 2012$ & 4532.4 \\
\hline $21 / 03 / 2012$ & 28/03/2012 & 4471.1 \\
\hline $04 / 04 / 2012$ & $11 / 04 / 2012$ & 4441.9 \\
\hline $18 / 04 / 2012$ & $25 / 04 / 2012$ & 4394.2 \\
\hline $02 / 05 / 2012$ & 09/05/2012 & 4416.0 \\
\hline $16 / 05 / 2012$ & $23 / 05 / 2012$ & 4405.2 \\
\hline $30 / 05 / 2012$ & 06/06/2012 & 4416.0 \\
\hline $13 / 06 / 2012$ & 20/06/2012 & 4385.3 \\
\hline $27 / 06 / 2012$ & 04/07/2012 & 3907.9 \\
\hline $11 / 07 / 2012$ & $18 / 07 / 2012$ & 4419.2 \\
\hline $25 / 07 / 2012$ & $01 / 08 / 2012$ & 4354.2 \\
\hline 08/08/2012 & $15 / 08 / 2012$ & 4423.9 \\
\hline $22 / 08 / 2012$ & 29/08/2012 & 4442.2 \\
\hline 05/09/2012 & $12 / 09 / 2012$ & 4405.4 \\
\hline 19/09/2012 & 26/09/2012 & 4432.3 \\
\hline 03/10/2012 & 10/10/2012 & 4452.7 \\
\hline $17 / 10 / 2012$ & $24 / 10 / 2012$ & 4464.8 \\
\hline $31 / 10 / 2012$ & 07/11/2012 & 4466.7 \\
\hline $14 / 11 / 2012$ & $21 / 11 / 2012$ & 3791.0 \\
\hline 28/11/2012 & $05 / 12 / 2012$ & 5055.8 \\
\hline $12 / 12 / 2012$ & $19 / 12 / 2012$ & 4533.8 \\
\hline $26 / 12 / 2012$ & $02 / 01 / 2013$ & 4550.3 \\
\hline 09/01/2013 & $16 / 01 / 2013$ & 4572.9 \\
\hline 23/01/2013 & $30 / 01 / 2013$ & 4478.6 \\
\hline $06 / 02 / 2013$ & $13 / 02 / 2013$ & 4510.9 \\
\hline 20/02/2013 & $27 / 02 / 2013$ & 4550.5 \\
\hline 06/03/2013 & $13 / 03 / 2013$ & 4425.3 \\
\hline 20/03/2013 & $27 / 03 / 2013$ & 4561.0 \\
\hline 03/04/2013 & $10 / 04 / 2013$ & 4484.9 \\
\hline $17 / 04 / 2013$ & $24 / 04 / 2013$ & 4435.5 \\
\hline 01/05/2013 & 08/05/2013 & 4420.2 \\
\hline $15 / 05 / 2013$ & $22 / 05 / 2013$ & 4355.1 \\
\hline $29 / 05 / 2013$ & $05 / 06 / 2013$ & 4421.9 \\
\hline $12 / 06 / 2013$ & $19 / 06 / 2013$ & 4364.2 \\
\hline 26/06/2013 & 03/07/2013 & 1929.2 \\
\hline $10 / 07 / 2013$ & $17 / 07 / 2013$ & 4393.7 \\
\hline 24/07/2013 & $31 / 07 / 2013$ & 3434.6 \\
\hline 07/08/2013 & $14 / 08 / 2013$ & 920.4 \\
\hline 21/08/2013 & 28/08/2013 & 4435.9 \\
\hline 04/09/2013 & $11 / 09 / 2013$ & 3050.3 \\
\hline $18 / 09 / 2013$ & $25 / 09 / 2013$ & 4453.2 \\
\hline $02 / 10 / 2013$ & 09/10/2013 & 4497.8 \\
\hline $16 / 10 / 2013$ & $23 / 10 / 2013$ & 4416.5 \\
\hline $30 / 10 / 2013$ & $06 / 11 / 2013$ & 4433.0 \\
\hline $13 / 11 / 2013$ & $20 / 11 / 2013$ & 4515.6 \\
\hline $27 / 11 / 2013$ & $04 / 12 / 2013$ & 4539.5 \\
\hline $11 / 12 / 2013$ & $18 / 12 / 2013$ & 4562.4 \\
\hline $25 / 12 / 2013$ & $01 / 01 / 2014$ & 4473.2 \\
\hline
\end{tabular}


Table S2: Air sample volumes collected for CUPs analysis from background site. Sample volumes were corrected for temperature and pressure.

\begin{tabular}{|c|c|c|}
\hline Sampling start & Sampling end & Sampling volume $\left(\mathrm{m}^{3}\right)$ \\
\hline $04 / 01 / 2012$ & $11 / 01 / 2012$ & 4529.2 \\
\hline $18 / 01 / 2012$ & $25 / 01 / 2012$ & 4540.0 \\
\hline $01 / 02 / 2012$ & 08/02/2012 & 4083.9 \\
\hline $15 / 02 / 2012$ & $22 / 02 / 2012$ & 4550.7 \\
\hline $29 / 02 / 2012$ & 07/03/2012 & 4509.3 \\
\hline $14 / 03 / 2012$ & $21 / 03 / 2012$ & 4487.2 \\
\hline 28/03/2012 & 04/04/2012 & 4455.0 \\
\hline $11 / 04 / 2012$ & $18 / 04 / 2012$ & 4423.1 \\
\hline $25 / 04 / 2012$ & $02 / 05 / 2012$ & 4387.0 \\
\hline 09/05/2012 & $16 / 05 / 2012$ & 4430.8 \\
\hline $23 / 05 / 2012$ & $30 / 05 / 2012$ & 4413.2 \\
\hline $06 / 06 / 2012$ & $13 / 06 / 2012$ & 4405.9 \\
\hline $20 / 06 / 2012$ & $27 / 06 / 2012$ & 4078.0 \\
\hline $04 / 07 / 2012$ & $11 / 07 / 2012$ & 3210.0 \\
\hline $18 / 07 / 2012$ & 25/07/2012 & 3042.6 \\
\hline $01 / 08 / 2012$ & 08/08/2012 & 4373.7 \\
\hline $15 / 08 / 2012$ & $22 / 08 / 2012$ & 4408.9 \\
\hline 29/08/2012 & 05/09/2012 & 4409.5 \\
\hline $12 / 09 / 2012$ & $19 / 09 / 2012$ & 4395.0 \\
\hline $26 / 09 / 2012$ & 03/10/2012 & 4436.4 \\
\hline $10 / 10 / 2012$ & $17 / 10 / 2012$ & 4465.4 \\
\hline $24 / 10 / 2012$ & $31 / 10 / 2012$ & 4503.7 \\
\hline 07/11/2012 & $14 / 11 / 2012$ & 4472.3 \\
\hline $22 / 11 / 2012$ & $28 / 11 / 2012$ & 3801.2 \\
\hline $05 / 12 / 2012$ & $12 / 12 / 2012$ & 4561.7 \\
\hline $19 / 12 / 2012$ & $26 / 12 / 2012$ & 4541.7 \\
\hline $02 / 01 / 2013$ & 09/01/2013 & 4545.5 \\
\hline $16 / 01 / 2013$ & $23 / 01 / 2013$ & 4504.0 \\
\hline $30 / 01 / 2013$ & $06 / 02 / 2013$ & 4483.7 \\
\hline $13 / 02 / 2013$ & $20 / 02 / 2013$ & 4541.4 \\
\hline $27 / 02 / 2013$ & $06 / 03 / 2013$ & 4524.2 \\
\hline $13 / 03 / 2013$ & $20 / 03 / 2013$ & 4505.0 \\
\hline $27 / 03 / 2013$ & 03/04/2013 & 4846.6 \\
\hline $10 / 04 / 2013$ & $17 / 04 / 2013$ & 4448.4 \\
\hline $24 / 04 / 2013$ & $01 / 05 / 2013$ & 4403.6 \\
\hline $08 / 05 / 2013$ & $15 / 05 / 2013$ & 4411.9 \\
\hline $22 / 05 / 2013$ & $29 / 05 / 2013$ & 4413.1 \\
\hline $05 / 06 / 2013$ & $12 / 06 / 2013$ & 4379.3 \\
\hline $19 / 06 / 2013$ & $26 / 06 / 2013$ & 4376.0 \\
\hline $03 / 07 / 2013$ & $10 / 07 / 2013$ & 4388.4 \\
\hline $17 / 07 / 2013$ & $24 / 07 / 2013$ & 4357.3 \\
\hline $31 / 07 / 2013$ & $07 / 08 / 2013$ & 576.7 \\
\hline $14 / 08 / 2013$ & $21 / 08 / 2013$ & 1886.4 \\
\hline $28 / 08 / 2013$ & $04 / 09 / 2013$ & 4432.6 \\
\hline $11 / 09 / 2013$ & $18 / 09 / 2013$ & 4452.3 \\
\hline $25 / 09 / 2013$ & $02 / 10 / 2013$ & 4473.0 \\
\hline 09/10/2013 & $16 / 10 / 2013$ & 4467.6 \\
\hline $23 / 10 / 2013$ & $30 / 10 / 2013$ & 4404.1 \\
\hline $06 / 11 / 2013$ & $13 / 11 / 2013$ & 4446.2 \\
\hline $20 / 11 / 2013$ & $27 / 11 / 2013$ & 4480.9 \\
\hline $04 / 12 / 2013$ & $11 / 12 / 2013$ & 4515.9 \\
\hline $18 / 12 / 2013$ & $25 / 12 / 2013$ & 4495.4 \\
\hline
\end{tabular}


Table S3: Cascade impactor air sample volumes collected for OCPs analysis. Sample volumes were corrected for temperature and pressure.

Start of sampling End of sampling Volume sampled $\left(\mathrm{m}^{3}\right)$

\begin{tabular}{|c|c|c|c|c|}
\hline \multirow{12}{*}{ Rural site } & \multirow{3}{*}{ Winter } & $6 / 1 / 2010$ & $13 / 1 / 2010$ & 11014 \\
\hline & & $3 / 2 / 2010$ & $9 / 2 / 2010$ & 9604 \\
\hline & & $5 / 3 / 2010$ & $12 / 3 / 2010$ & 11424 \\
\hline & \multirow{3}{*}{ Spring } & $9 / 4 / 2010$ & $16 / 4 / 2010$ & 11162 \\
\hline & & $7 / 5 / 2010$ & $10 / 5 / 2010$ & 2397 \\
\hline & & 7/6/2010 & $14 / 6 / 2010$ & 11103 \\
\hline & \multirow{3}{*}{ Summer } & $5 / 7 / 2010$ & $11 / 7 / 2010$ & 5957 \\
\hline & & $2 / 8 / 2010$ & $9 / 8 / 2010$ & 11425 \\
\hline & & 9/9/2010 & $15 / 9 / 2010$ & 8313 \\
\hline & \multirow{3}{*}{ Autumn } & $30 / 10 / 2009$ & $3 / 11 / 2009$ & 6659 \\
\hline & & $20 / 11 / 2009$ & $27 / 11 / 2009$ & 11424 \\
\hline & & $8 / 10 / 2010$ & $15 / 10 / 2010$ & 7297 \\
\hline \multirow{12}{*}{ Urban site } & \multirow{3}{*}{ Winter } & $6 / 1 / 2010$ & $13 / 1 / 2010$ & 11141 \\
\hline & & $3 / 2 / 2010$ & $10 / 2 / 2010$ & 11364 \\
\hline & & $3 / 3 / 2010$ & $10 / 3 / 2010$ & 11316 \\
\hline & \multirow{3}{*}{ Spring } & $9 / 4 / 2010$ & $16 / 4 / 2010$ & 10915 \\
\hline & & $7 / 5 / 2010$ & $10 / 5 / 2010$ & 4293 \\
\hline & & $14 / 6 / 2010$ & $21 / 6 / 2010$ & 11245 \\
\hline & \multirow{3}{*}{ Summer } & $12 / 7 / 2010$ & $19 / 7 / 2010$ & 11420 \\
\hline & & $16 / 8 / 2010$ & $23 / 8 / 2010$ & 10775 \\
\hline & & $15 / 9 / 2010$ & $18 / 9 / 2010$ & 4755 \\
\hline & \multirow{3}{*}{ Autumn } & $30 / 10 / 2009$ & $2 / 11 / 2009$ & 4909 \\
\hline & & $20 / 11 / 2009$ & $27 / 11 / 2009$ & 10964 \\
\hline & & $8 / 10 / 2010$ & $15 / 10 / 2010$ & 10863 \\
\hline
\end{tabular}


Table S4: Cascade impactor air sample volumes collected for CUPs analysis. Sample volumes were corrected for temperature and pressure.

\begin{tabular}{|c|c|c|c|c|}
\hline & & Start of sampling & End of sampling & Volume sampled $\left(\mathrm{m}^{3}\right)$ \\
\hline \multirow{11}{*}{ Rural site } & \multirow{3}{*}{ Winter } & $27 / 11 / 2009$ & $4 / 12 / 2009$ & 11425 \\
\hline & & $13 / 1 / 2010$ & $20 / 1 / 2010$ & 11289 \\
\hline & & $10 / 2 / 2010$ & $13 / 2 / 2010$ & 4443 \\
\hline & \multirow{3}{*}{ Spring } & $12 / 3 / 2010$ & $19 / 3 / 2010$ & 11424 \\
\hline & & $16 / 4 / 2010$ & $23 / 4 / 2010$ & 11347 \\
\hline & & $13 / 5 / 2010$ & $20 / 5 / 2010$ & 11425 \\
\hline & \multirow{3}{*}{ Summer } & $14 / 6 / 2010$ & $21 / 6 / 2010$ & 11258 \\
\hline & & $12 / 7 / 2010$ & $19 / 7 / 2010$ & 10529 \\
\hline & & $16 / 8 / 2010$ & $23 / 8 / 2010$ & 9770 \\
\hline & \multirow{2}{*}{ Autumn } & $9 / 10 / 2009$ & $16 / 10 / 2009$ & 11353 \\
\hline & & $16 / 9 / 2010$ & $23 / 9 / 2010$ & 11425 \\
\hline \multirow{11}{*}{ Urban site } & \multirow{3}{*}{ Winter } & $27 / 11 / 2009$ & $4 / 12 / 2009$ & 11323 \\
\hline & & $13 / 1 / 2010$ & $20 / 1 / 2010$ & 11283 \\
\hline & & $10 / 2 / 2010$ & $17 / 2 / 2010$ & 11411 \\
\hline & \multirow{3}{*}{ Spring } & $10 / 3 / 2010$ & $17 / 3 / 2010$ & 11424 \\
\hline & & $16 / 4 / 2010$ & $22 / 4 / 2010$ & 9453 \\
\hline & & $21 / 5 / 2010$ & $28 / 5 / 2010$ & 11371 \\
\hline & \multirow{3}{*}{ Summer } & $22 / 6 / 2010$ & $25 / 6 / 2010$ & 6049 \\
\hline & & $19 / 7 / 2010$ & $26 / 7 / 2010$ & 11117 \\
\hline & & $23 / 8 / 2010$ & $26 / 8 / 2010$ & 5005 \\
\hline & \multirow{2}{*}{ Autumn } & $9 / 10 / 2009$ & $16 / 10 / 2009$ & 11181 \\
\hline & & $22 / 9 / 2010$ & $29 / 9 / 2010$ & 10700 \\
\hline
\end{tabular}


Table S5: Selected LC-MS/MS experimental parameters, limits of detection (LODs) and limits of quantification (LOQs) (SRM indicates "Selected Reaction Monitoring" and $\mathrm{R}_{t}$ indicates "Retention time").

\begin{tabular}{ccccccc} 
Analyte & $\mathrm{m} / \mathrm{z}$ & $\begin{array}{c}\mathrm{SRM} \\
1\end{array}$ & $\begin{array}{c}\mathrm{SRM} \\
2\end{array}$ & $\begin{array}{c}\mathrm{R}_{\mathrm{t}} \\
(\mathrm{min})\end{array}$ & $\begin{array}{c}\mathrm{LOD} \\
(\mathrm{ng} / \mathrm{mL})\end{array}$ & $\begin{array}{c}\mathrm{LOQ} \\
(\mathrm{ng} / \mathrm{mL})\end{array}$ \\
\hline Acetochlor & 270.1 & 224.2 & 148.1 & 4.2 & 0.1 & 0.25 \\
Alachlor & 270.1 & 238.1 & 162.1 & 4.2 & 0.5 & 1 \\
Atrazine & 216.1 & 174.2 & 68.0 & 3.5 & 0.05 & 0.1 \\
Azinphos methyl & 318.0 & 160.0 & 132.0 & 3.7 & 0.1 & 0.25 \\
Carbendazim & 192.0 & 160.0 & 131.9 & 2.9 & 0.03 & 0.05 \\
Chlorpyrifos & 349.9 & 96.9 & 197.9 & 6.1 & 0.25 & 0.5 \\
Chlorotoluron & 213.1 & 72.2 & 46.2 & 3.4 & 0.25 & 0.5 \\
Diazinon & 305.0 & 169.0 & 96.9 & 4.6 & 0.01 & 0.25 \\
Dimethachlor & 256.1 & 224.0 & 148.1 & 3.5 & 0.2 & 0.5 \\
Dimethoate & 230.0 & 198.9 & 124.9 & 2.8 & 0.01 & 0.03 \\
Disulfoton & 275.0 & 88.9 & 61.0 & 4.8 & 0.01 & 0.02 \\
Diuron & 232.9 & 71.8 & 46.1 & 3.5 & 0.1 & 0.25 \\
Fenitrothion & 278.0 & 124.9 & 108.9 & 4.0 & 5 & 20 \\
Fenpropimorph & 304.2 & 147.0 & 117.0 & 8.2 & 0.02 & 0.05 \\
Fonofos & 247.0 & 109.0 & 136.9 & 4.6 & 0.1 & 0.25 \\
Isoproturon & 207.2 & 72.1 & 46.1 & 3.5 & 0.06 & 0.12 \\
Malathion & 331.0 & 127.0 & 99.0 & 3.9 & 0.25 & 0.5 \\
Metamitron & 203.0 & 175.1 & 104.0 & 2.8 & 0.25 & 0.5 \\
Metazachlor & 278.2 & 134.2 & 210.2 & 3.8 & 0.05 & 0.1 \\
Metribuzin & 215.1 & 187.1 & 84.0 & 3.2 & 0.1 & 0.25 \\
Prochloraz & 376.0 & 308.0 & 70.0 & 4.7 & 0.01 & 0.03 \\
Pyrazon & 222.0 & 104.0 & 77.0 & 2.8 & 0.06 & 0.12 \\
Simazine & 202.0 & 132.0 & 124.1 & 3.2 & 0.05 & 0.1 \\
S-metolachlor & 284.1 & 252.2 & 176.1 & 4.2 & 0.1 & 0.25 \\
Temephos & 466.9 & 419.0 & 124.9 & 5.7 & 0.03 & 0.05 \\
Terbufos & 289.0 & 103.0 & 232.9 & 5.7 & 0.05 & 0.1 \\
Terbuthylazine & 230.1 & 174.0 & 67.9 & 3.9 & 0.05 & 0.1 \\
\hline & & & & & & \\
\hline
\end{tabular}




\section{Breakthrough}

Breakthrough of gas-phase compounds was evaluated by quantifying separately each of the two PUFs placed in series for all the weekly air samples (sample volume $=3042-4561 \mathrm{~m}^{3}$ for CUPs and $3791-8103$ $\mathrm{m}^{3}$ for OCPs) collected at the background site in $2012(\mathrm{~N}=52)$. This covered a large range of meteorological conditions, and the results are considered applicable to 2013. Summary of the results of the breakthrough experiments is presented in Tables S5 and S6. Based on these results, the current sampling configuration with two PUFs in series is considered to be efficient for trapping the selected OCPs and CUPs in the gas phase.

Table S6: Results of breakthrough experiments for CUPs. N/D indicates compounds that were not detected.

\begin{tabular}{|c|c|c|c|c|c|c|}
\hline \multirow[b]{2}{*}{ Compound } & \multirow{2}{*}{$\begin{array}{c}\text { Frequency of } \\
\text { detection on } \\
\text { upper PUF }\end{array}$} & \multirow{2}{*}{$\begin{array}{c}\text { Frequency of } \\
\text { detection on } \\
\text { lower PUF }\end{array}$} & \multicolumn{4}{|c|}{$\%$ of compound mass found on the lower PUF } \\
\hline & & & Min & Max & Average & $\begin{array}{l}\text { Standard } \\
\text { deviation }\end{array}$ \\
\hline Acetochlor & $50 \%$ & $27 \%$ & 0 & 34.5 & 10.4 & 13.6 \\
\hline Alachlor & $3.8 \%$ & $0 \%$ & 0 & 0 & 0 & $\mathrm{~N} / \mathrm{D}$ \\
\hline Atrazine & $3.8 \%$ & $0 \%$ & 0 & 0 & 0 & $\mathrm{~N} / \mathrm{D}$ \\
\hline Azinphos methyl & $0 \%$ & $0 \%$ & N/D & N/D & N/D & N/D \\
\hline Carbendazim & $0 \%$ & $3.8 \%$ & 100 & 100 & 100 & $\mathrm{~N} / \mathrm{D}$ \\
\hline Chlorpyrifos & $92 \%$ & $35 \%$ & 0 & 22.9 & 2.86 & 5.95 \\
\hline Chlorotoluron & $3.8 \%$ & $0 \%$ & 0 & 0 & 0 & N/D \\
\hline Diazinon & $3.8 \%$ & $0 \%$ & 0 & 0 & 0 & N/D \\
\hline Dimethachlor & $38 \%$ & $15 \%$ & 0 & 24.6 & 3.38 & 7.69 \\
\hline Dimethoate & $0 \%$ & $0 \%$ & N/D & N/D & $\mathrm{N} / \mathrm{D}$ & $\mathrm{N} / \mathrm{D}$ \\
\hline Disulfoton & $0 \%$ & $0 \%$ & N/D & N/D & $\mathrm{N} / \mathrm{D}$ & N/D \\
\hline Diuron & $0 \%$ & $0 \%$ & N/D & N/D & N/D & N/D \\
\hline Fenitrothion & $0 \%$ & $0 \%$ & N/D & N/D & N/D & N/D \\
\hline Fenpropimorph & $0 \%$ & $3.8 \%$ & 100 & 100 & 100 & N/D \\
\hline Fonofos & $0 \%$ & $0 \%$ & N/D & N/D & N/D & N/D \\
\hline Isoproturon & $54 \%$ & $3.8 \%$ & 0 & 50.2 & 3.59 & 13.4 \\
\hline Malathion & $0 \%$ & $0 \%$ & N/D & N/D & N/D & N/D \\
\hline Metamitron & $15 \%$ & $3.8 \%$ & 0 & 100 & 20.0 & 44.7 \\
\hline Metazachlor & $69 \%$ & $19 \%$ & 0 & 21.6 & 1.48 & 5.14 \\
\hline Metribuzin & $7.7 \%$ & $3.8 \%$ & 0 & 100 & 33.3 & 57.7 \\
\hline Prochloraz & $0 \%$ & $0 \%$ & N/D & N/D & N/D & N/D \\
\hline Pyrazon & $0 \%$ & $0 \%$ & $\mathrm{~N} / \mathrm{D}$ & N/D & N/D & N/D \\
\hline Simazine & $0 \%$ & $0 \%$ & N/D & N/D & N/D & N/D \\
\hline S-metolachlor & $69 \%$ & $23 \%$ & 0 & 11.0 & 1.16 & 2.71 \\
\hline Temephos & $0 \%$ & $0 \%$ & N/D & N/D & N/D & N/D \\
\hline Terbufos & $0 \%$ & $0 \%$ & N/D & N/D & N/D & N/D \\
\hline Terbuthylazine & $73 \%$ & $23 \%$ & 0 & 3.34 & 0.415 & 0.879 \\
\hline
\end{tabular}


Table S7: Results of breakthrough experiments for OCPs.

\begin{tabular}{ccccccc} 
& $\begin{array}{c}\text { Detection } \\
\text { frequency } \\
\text { on upper }\end{array}$ & $\begin{array}{c}\text { Detection } \\
\text { frequency } \\
\text { on lower }\end{array}$ & \multicolumn{3}{c}{ \% of compound mass found on the lower PUF } \\
\cline { 5 - 7 } & PUF & PUF & Min & Max & Average & $\begin{array}{c}\text { Standard } \\
\text { deviation }\end{array}$ \\
\hline$\alpha-\mathrm{HCH}$ & $100 \%$ & $100 \%$ & 0.04 & 65.6 & 35.4 & 17.0 \\
$\beta-\mathrm{HCH}$ & $100 \%$ & $88 \%$ & 0 & 26.6 & 9.97 & 8.81 \\
$\gamma-\mathrm{HCH}$ & $100 \%$ & $100 \%$ & 1.03 & 49.3 & 21.9 & 18.0 \\
$\delta$-HCH & $100 \%$ & $96 \%$ & 0 & 66.4 & 22.8 & 15.7 \\
$o, p^{\prime}$-DDE & $100 \%$ & 465 & 0 & 21.8 & 1.78 & 5.03 \\
$p, p^{\prime}$-DDE & $100 \%$ & $85 \%$ & 0 & 22.0 & 2.01 & 5.03 \\
$o, p^{\prime}$-DDD & $100 \%$ & $35 \%$ & 0 & 20.4 & 1.76 & 5.00 \\
$p, p^{\prime}$-DDD & $100 \%$ & $77 \%$ & 0 & 18.6 & 2.22 & 4.58 \\
$o, p^{\prime}$-DDT & $100 \%$ & $73 \%$ & 0 & 19.3 & 1.61 & 4.23 \\
$p, p^{\prime}$-DDT & $100 \%$ & $65 \%$ & 0 & 16.1 & 1.40 & 3.50 \\
\hline
\end{tabular}

Table S8: Recoveries (in \%) and standard deviations (SD) of individual OCPs. Average recoveries were calculated from spike and recovery tests of PUFs $(n=3)$.

\begin{tabular}{ccc} 
Compound & Average recovery (\%) & SD \\
\hline$\alpha-\mathrm{HCH}$ & 94.2 & 6.31 \\
$\beta-\mathrm{HCH}$ & 91.2 & 6.10 \\
$\gamma-\mathrm{HCH}$ & 94.4 & 6.86 \\
$\delta$-HCH & 99.1 & 7.83 \\
o,p'-DDE & 88.7 & 5.69 \\
p,p'-DDE & 87.2 & 6.26 \\
o,p'-DDD & 93.4 & 7.14 \\
p,p'-DDD & 91.4 & 8.28 \\
o,p'-DDT & 104.9 & 5.44 \\
p,p'-DDT & 113.8 & 6.10
\end{tabular}


Table S9: Recoveries (in \%) and standard deviations (SD) of individual CUPs. Average recoveries were calculated from spike and recovery tests of air sampling media (filters and PUFs, $n=10$ ).

\begin{tabular}{|c|c|c|c|c|}
\hline \multirow[b]{2}{*}{ Compound } & \multicolumn{2}{|c|}{ QFFs } & \multicolumn{2}{|c|}{ PUFs } \\
\hline & $\begin{array}{c}\text { Average } \\
\text { recovery } \\
(\%)\end{array}$ & $\mathrm{SD}$ & $\begin{array}{c}\text { Average } \\
\text { recovery } \\
(\%)\end{array}$ & SD \\
\hline Acetochlor & 97.2 & 13.2 & 93.2 & 17.3 \\
\hline Alachlor & 81.4 & 6.05 & 115 & 17.4 \\
\hline Atrazine & 90.5 & 9.67 & 82 & 15.8 \\
\hline Azinphos methyl & 64.4 & 12.7 & 68.5 & 15.7 \\
\hline Carbendazim & 58.1 & 4.45 & 83.3 & 9.6 \\
\hline Chlorotoluron & 65.9 & 14.6 & 100 & 16.8 \\
\hline Chlorpyrifos & 79.5 & 6.68 & 111 & 17.3 \\
\hline Diazinon & 103 & 21.7 & 18.9 & 12.9 \\
\hline Dimethachlor & 74.8 & 6.76 & 86.1 & 16 \\
\hline Dimethoate & 77 & 8.44 & 98.1 & 15.8 \\
\hline Disulfoton & 90.2 & 17.3 & 52.4 & 21.4 \\
\hline Diuron & 59.8 & 14.4 & 102 & 13.9 \\
\hline Fenitrothion & 81.2 & 36.5 & 56.3 & 19.5 \\
\hline Fenpropimorph & 80.3 & 14.2 & 103.6 & 16.7 \\
\hline Fonofos & 90.4 & 21.7 & 114 & 14.8 \\
\hline Isoproturon & 113 & 23.7 & 109 & 17.8 \\
\hline Malathion & 55.2 & 7.14 & 58 & 18.1 \\
\hline Metamitron & 83.4 & 7.38 & 81.2 & 12.3 \\
\hline Metazachlor & 58.9 & 9.48 & 107.1 & 18.7 \\
\hline Metribuzin & 102 & 21.8 & 52.8 & 15.9 \\
\hline Prochloraz & 81.5 & 12.2 & 78 & 16.7 \\
\hline Pyrazon & 90.5 & 4.11 & 104 & 17.6 \\
\hline Simazine & 80.7 & 8.3 & 81.3 & 15.5 \\
\hline S-metolachlor & 109 & 10.1 & 109.8 & 19.2 \\
\hline Temephos & 60.3 & 18.2 & 85.6 & 15.9 \\
\hline Terbufos & 84.9 & 5.56 & 66.3 & 16.6 \\
\hline Terbuthylazine & 70.5 & 7.65 & 96 & 15.6 \\
\hline
\end{tabular}


Table S10: Use of agricultural pesticides in the Czech Republic in 2012 (in kg) (SRS, 2013).

\begin{tabular}{|c|c|c|c|c|c|c|c|c|c|c|c|c|c|}
\hline $\begin{array}{l}\bar{\Xi} \\
\overline{0} \\
\stackrel{\Xi}{0} \\
0\end{array}$ & సีّ & 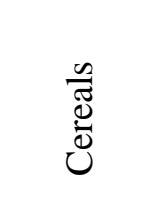 & $\stackrel{\stackrel{N}{N}}{\sum_{\Sigma}^{*}}$ & 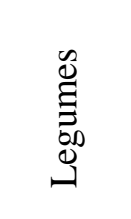 & 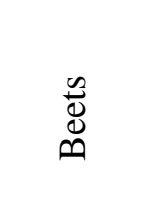 & 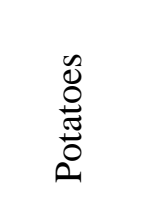 & 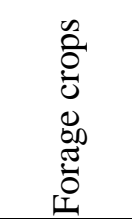 & $\frac{\ddot{a}}{\frac{a}{a}}$ & $\stackrel{2}{0}$ & $\begin{array}{l}\frac{0}{0} \\
\frac{\pi}{0} \\
\frac{\pi}{0} \\
\infty \\
\infty \\
0\end{array}$ & 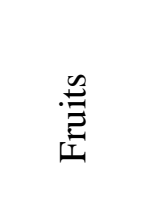 & 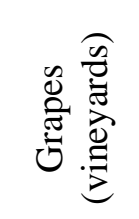 & $\frac{\tilde{b}}{\tilde{b}}$ \\
\hline Acetochlor & 173604 & 93 & 151815 & 3 & 0 & 0 & 0 & 21693 & 0 & 0 & 0 & 0 & 0 \\
\hline Carbendazim & 42392 & 19018 & 0 & 6 & 855 & 4 & 0 & 22467 & 0 & 43 & 0 & 0 & 0 \\
\hline Chlorpyrifos & 187245 & 41511 & 3124 & 3241 & 6779 & 1187 & 175 & 131217 & 0 & 0 & 8 & 0 & 3 \\
\hline Chlorotoluron & 91315 & 89533 & 67 & 0 & 19 & 0 & 59 & 1637 & 0 & 0 & 0 & 0 & 0 \\
\hline Dimethachlor & 54927 & 352 & 0 & 0 & 0 & 0 & 0 & 54574 & 0 & 0 & 0 & 0 & 0 \\
\hline Dimethoate & 883 & 815 & 0 & 0 & 29 & 36 & 0 & 2 & 0 & 1 & 0 & 0 & 0 \\
\hline Fenpropimorph & 66599 & 63116 & 0 & 0 & 3480 & 0 & 3 & 0 & 0 & 0 & 0 & 0 & 0 \\
\hline Isoproturon & 141164 & 140976 & 9 & 0 & 33 & 0 & 2 & 145 & 0 & 0 & 0 & 0 & 0 \\
\hline Metamitron & 94564 & 0 & 0 & 0 & 94123 & 0 & 8 & 426 & 0 & 5 & 3 & 0 & 0 \\
\hline Metazachlor & 186977 & 444 & 0 & 0 & 0 & 3 & 0 & 185957 & 0 & 571 & 0 & 0 & 3 \\
\hline Metribuzin & 4579 & 0 & 0 & 94 & 0 & 4324 & 49 & 0 & 0 & 111 & 0 & 0 & 0 \\
\hline Prochloraz & 148446 & 117271 & 0 & 0 & 7789 & 0 & 0 & 23386 & 0 & 0 & 0 & 0 & 0 \\
\hline Pyrazon & 40359 & 1 & 0 & 0 & 40358 & 0 & 0 & 0 & 0 & 0 & 0 & 0 & 0 \\
\hline S-Metolachlor & 76871 & 244 & 72959 & 101 & 2077 & 348 & 0 & 1143 & 0 & 0 & 0 & 0 & 0 \\
\hline Terbuthylazine & 111639 & 228 & 111346 & 0 & 0 & 0 & 0 & 65 & 0 & 0 & 0 & 0 & 0 \\
\hline $\begin{array}{l}\text { Total (selected } \\
\text { pesticides) }\end{array}$ & 1421563 & 473603 & 339320 & 3444 & 155541 & 5901 & 297 & 442712 & 0 & 730 & 11 & 0 & 6 \\
\hline $\begin{array}{c}\text { Total (all } \\
\text { pesticides) }\end{array}$ & 5701697 & 2639442 & 599455 & 35410 & 318712 & 104919 & 43275 & 1416009 & 88792 & 27102 & 229389 & 169419 & 29773 \\
\hline
\end{tabular}


Table S11: Use of agricultural pesticides in the Czech Republic in 2013 (in kg) (SRS, 2014).

\begin{tabular}{|c|c|c|c|c|c|c|c|c|c|c|c|c|c|}
\hline 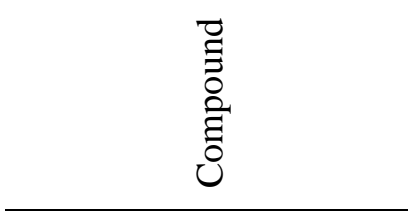 & $\stackrel{\pi}{0}$ & 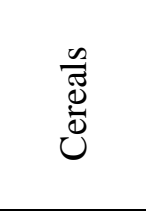 & 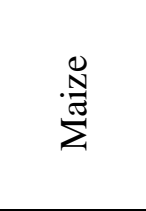 & 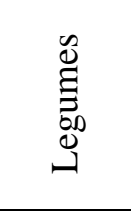 & $\begin{array}{l}\stackrel{\infty}{\Delta} \\
\infty \\
\infty\end{array}$ & $\begin{array}{l}\mathscr{0} \\
\stackrel{0}{\pi} \\
0 \\
0\end{array}$ & 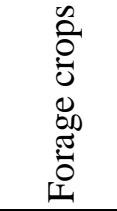 & $\begin{array}{l}\frac{0}{0} \\
\frac{a}{2} \\
\frac{0}{0}\end{array}$ & O̊̊̆ & $\begin{array}{l}\frac{0}{0} \\
\frac{\pi}{0} \\
\frac{\pi}{0} \\
\sum^{\infty}\end{array}$ & 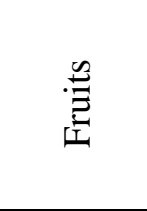 & 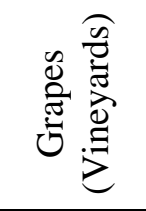 & $\frac{\mathscr{\Delta}}{\stackrel{\Xi}{\Xi}}$ \\
\hline Acetochlor & 101053 & 256 & 91002 & 0 & 0 & 0 & 0 & 9795 & 0 & 0 & 0 & 0 & 0 \\
\hline Carbendazim & 31050 & 11186 & 16 & 0 & 701 & 0 & 0 & 19099 & 0 & 48 & 0 & 0 & 0 \\
\hline Chlorotoluron & 107418 & 105063 & 45 & 0 & 0 & 0 & 37 & 2273 & 0 & 0 & 0 & 0 & 0 \\
\hline Chlorpyrifos & 178363 & 29436 & 2111 & 3026 & 5525 & 1125 & 160 & 136915 & 0 & 65 & 0 & 0 & 0 \\
\hline Dimethachlor & 44101 & 5 & 0 & 92 & 0 & 0 & 0 & 43783 & 0 & 191 & 0 & 0 & 30 \\
\hline Dimethoate & 147 & 20 & 0 & 0 & 82 & 45 & 0 & 0 & 0 & 1 & 0 & 0 & 0 \\
\hline Fenpropimorph & 79620 & 75795 & 2 & 0 & 3790 & 0 & 15 & 19 & 0 & 0 & 0 & 0 & 0 \\
\hline Isoproturon & 94004 & 93858 & 26 & 0 & 0 & 0 & 80 & 40 & 0 & 0 & 0 & 0 & 0 \\
\hline Metamitron & 94347 & 49 & 0 & 0 & 94158 & 0 & 0 & 0 & 0 & 45 & 0 & 0 & 96 \\
\hline Metazachlor & 169986 & 393 & 0 & 0 & 35 & 0 & 0 & 168788 & 0 & 750 & 0 & 0 & 19 \\
\hline Metribuzin & 4606 & 0 & 5 & 23 & 0 & 4488 & 23 & 10 & 0 & 42 & 0 & 0 & 16 \\
\hline Prochloraz & 176504 & 143665 & 9 & 0 & 4636 & 0 & 0 & 28125 & 0 & 0 & 0 & 0 & 70 \\
\hline Pyrazon & 43372 & 0 & 0 & 0 & 43229 & 0 & 0 & 0 & 0 & 34 & 0 & 0 & 109 \\
\hline S-Metolachlor & 68577 & 1073 & 64090 & 33 & 1990 & 315 & 0 & 1076 & 0 & 1 & 0 & 0 & 0 \\
\hline Terbuthylazine & 113124 & 802 & 112102 & 0 & 0 & 0 & 0 & 195 & 0 & 1 & 0 & 0 & 24 \\
\hline Total (selected pesticides) & 1306270 & 461600 & 269408 & 3174 & 154146 & 5973 & 316 & 410116 & 0 & 1176 & 0 & 0 & 362 \\
\hline Total (all pesticides) & 5511032 & 2480955 & 560947 & 30239 & 299283 & 107638 & 36680 & 1402589 & 81403 & 28488 & 252425 & 208568 & 21820 \\
\hline
\end{tabular}


Table S12: Concentration of individual CUPs (in pg. $\mathrm{m}^{-3}$ ) in the gaseous phase at the background site. N/D indicates compounds that were not detected, N/A those that were not analyzed, and <LOQ indicates samples that were below the limit of quantification (in Table S7).

\begin{tabular}{|c|c|c|c|c|c|c|c|c|c|c|c|c|c|c|c|}
\hline 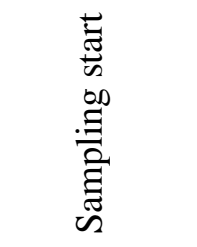 & 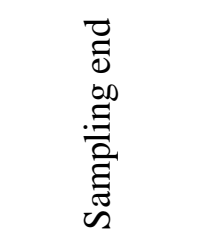 & 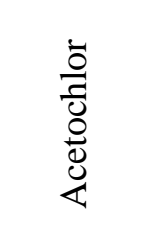 & $\begin{array}{l}\frac{\dot{0}}{\frac{\pi}{0}} \\
\frac{\pi}{2} \\
\frac{\pi}{2}\end{array}$ & 导 & 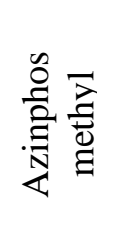 & 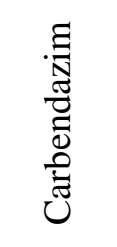 & $\begin{array}{l}\tilde{0} \\
\vdots \\
0 \\
0 \\
0 \\
0 \\
\frac{0}{0} \\
0\end{array}$ & 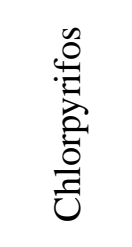 & 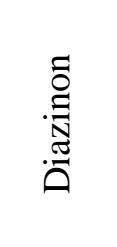 & 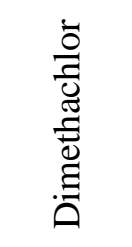 & 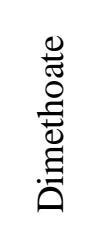 & 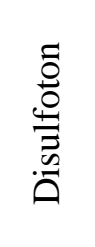 & $\begin{array}{l}\tilde{0} \\
\stackrel{\Xi}{\Xi} \\
.0\end{array}$ & 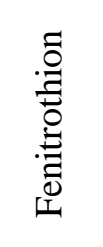 & 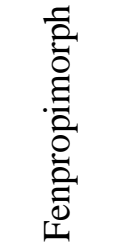 \\
\hline 4.1 .2012 & 11.1 .2012 & N/D & N/D & N/D & N/D & N/D & N/D & 0.119 & N/D & N/D & N/D & N/D & N/D & N/D & N/D \\
\hline 18.1 .2012 & 25.1 .2012 & N/D & N/D & N/D & N/D & N/D & N/D & 0.260 & N/D & N/D & N/D & N/D & N/D & N/D & N/D \\
\hline 1.2 .2012 & 8.2 .2012 & N/D & N/D & N/D & N/D & N/D & N/D & N/D & N/D & N/D & N/D & $\mathrm{N} / \mathrm{D}$ & N/D & N/D & $\mathrm{N} / \mathrm{D}$ \\
\hline 15.2.2012 & 22.2.2012 & N/D & N/D & N/D & N/D & N/D & N/D & 0.128 & N/D & N/D & N/D & N/D & $\mathrm{N} / \mathrm{D}$ & N/D & N/D \\
\hline 29.2.2012 & 7.3.2012 & N/D & N/D & N/D & N/D & N/D & N/D & 0.517 & N/D & N/D & N/D & N/D & N/D & N/D & N/D \\
\hline 14.3 .2012 & 21.3 .2012 & N/D & N/D & N/D & N/D & N/D & N/D & $<\mathrm{LOQ}$ & N/D & N/D & N/D & N/D & N/D & N/D & N/D \\
\hline 28.3.2012 & 4.4.2012 & N/D & N/D & N/D & N/D & N/D & N/D & 18.635 & N/D & N/D & N/D & N/D & N/D & N/D & N/D \\
\hline 11.4 .2012 & 18.4 .2012 & 10.197 & N/D & N/D & N/D & N/D & N/D & 31.200 & N/D & N/D & N/D & N/D & N/D & N/D & N/D \\
\hline 25.4 .2012 & 2.5.2012 & 30.134 & N/D & N/D & N/D & N/D & N/D & 6.218 & N/D & 0.944 & N/D & N/D & N/D & N/D & N/D \\
\hline 9.5 .2012 & 16.5 .2012 & 157.828 & $<\mathrm{LOQ}$ & N/D & N/D & N/D & N/D & 2.753 & $\mathrm{~N} / \mathrm{D}$ & 0.422 & N/D & N/D & N/D & N/D & N/D \\
\hline 23.5.2012 & 30.5 .2012 & 24.379 & 0.231 & 0.757 & N/D & N/D & N/D & 2.148 & N/D & 0.134 & N/D & N/D & N/D & N/D & N/D \\
\hline 6.6 .2012 & 13.6 .2012 & 33.367 & $<\mathrm{LOQ}$ & N/D & N/D & 0.223 & $\mathrm{~N} / \mathrm{D}$ & 3.155 & $\mathrm{~N} / \mathrm{D}$ & $<\mathrm{LOQ}$ & N/D & N/D & N/D & N/D & $<\mathrm{LOQ}$ \\
\hline 20.6 .2012 & 27.6.2012 & 8.646 & N/D & N/D & N/D & N/D & 0.390 & 7.540 & N/D & 0.220 & N/D & N/D & N/D & N/D & N/D \\
\hline 4.7.2012 & 11.7.2012 & 3.003 & N/D & N/D & N/D & N/D & N/D & 6.732 & N/D & N/D & N/D & N/D & N/D & N/D & 1.271 \\
\hline 18.7.2012 & 25.7 .2012 & 0.670 & N/D & N/D & N/D & N/D & N/D & 7.418 & N/D & N/D & N/D & N/D & N/D & N/D & N/D \\
\hline 1.8.2012 & 8.8.2012 & 0.474 & N/D & N/D & N/D & N/D & N/D & 1.877 & N/D & N/D & N/D & N/D & N/D & N/D & N/D \\
\hline 15.8 .2012 & 22.8.2012 & 0.152 & N/D & N/D & N/D & N/D & N/D & 0.148 & N/D & 47.699 & N/D & N/D & N/D & N/D & N/D \\
\hline 29.8 .2012 & 5.9.2012 & 0.730 & N/D & N/D & N/D & N/D & N/D & 6.047 & 0.177 & 70.805 & N/D & N/D & N/D & N/D & N/D \\
\hline 12.9 .2012 & 19.9.2012 & N/D & N/D & N/D & N/D & N/D & N/D & 1.515 & N/D & 6.497 & N/D & N/D & N/D & N/D & N/D \\
\hline 26.9 .2012 & 3.10 .2012 & 0.651 & $<\mathrm{LOQ}$ & N/D & $\mathrm{N} / \mathrm{D}$ & N/D & N/D & 11.094 & N/D & 1.501 & N/D & $\mathrm{N} / \mathrm{D}$ & N/D & N/D & N/D \\
\hline 10.10 .2012 & 17.10 .2012 & 0.157 & N/D & N/D & N/D & N/D & N/D & 22.842 & N/D & 0.425 & N/D & N/D & N/D & N/D & N/D \\
\hline 24.10.2012 & 31.10 .2012 & N/D & N/D & N/D & N/D & N/D & N/D & 64.355 & N/D & 0.133 & N/D & N/D & N/D & N/D & N/D \\
\hline 7.11.2012 & 14.11.2012 & N/D & N/D & N/D & N/D & N/D & N/D & 17.105 & N/D & N/D & N/D & N/D & N/D & N/D & N/D \\
\hline
\end{tabular}




\begin{tabular}{|c|c|c|c|c|c|c|c|c|c|c|c|c|c|c|c|}
\hline 22.11 .2012 & 28.11 .2012 & N/D & N/D & N/D & N/D & N/D & N/D & 6.814 & N/D & N/D & N/D & N/D & N/D & N/D & N/D \\
\hline 5.12 .2012 & 12.12.2012 & N/D & N/D & N/D & N/D & N/D & N/D & 0.212 & N/D & N/D & N/D & N/D & N/D & N/D & N/D \\
\hline 19.12.2012 & 26.12.2012 & N/D & N/D & N/D & N/D & N/D & N/D & 1.519 & N/D & N/D & N/D & N/D & N/D & N/D & N/D \\
\hline 2.1 .2013 & 9.1 .2013 & N/D & N/D & N/D & N/D & N/D & N/D & 0.814 & N/D & N/D & N/D & N/D & N/D & N/D & N/D \\
\hline 16.1 .2013 & 23.1 .2013 & N/D & N/D & N/D & N/D & N/D & N/D & $<\mathrm{LOQ}$ & N/D & N/D & N/D & N/D & N/D & N/D & N/D \\
\hline 30.1 .2013 & 6.2 .2013 & N/A & N/A & N/A & N/A & N/A & N/A & N/A & N/A & N/A & N/A & N/A & N/A & N/A & N/A \\
\hline 13.2 .2013 & 20.2.2013 & N/D & N/D & N/D & N/D & N/D & N/D & N/D & N/D & N/D & N/D & N/D & N/D & N/D & N/D \\
\hline 27.2.2013 & 6.3 .2013 & N/D & N/D & N/D & N/D & N/D & N/D & $\mathrm{N} / \mathrm{D}$ & N/D & $\mathrm{N} / \mathrm{D}$ & N/D & N/D & N/D & N/D & N/D \\
\hline 13.3 .2 & 20.3.2013 & N/D & N/D & N/D & N/D & N/D & N/D & $<\mathrm{LOQ}$ & N/D & N/D & N/D & N/D & N/D & N/D & N/D \\
\hline 27.3.2013 & 3.4 .2013 & N/D & N/D & N/D & N/D & N/D & N/D & N/D & N/D & N/D & N/D & N/D & N/D & N/D & 0.042 \\
\hline 10.4 . & 17.4 .2013 & N/D & N/D & N/D & N/D & N/D & N/D & 1.538 & N/D & N/D & N/D & N/D & N/D & N/D & 0.103 \\
\hline 24.4 & 1.5. & 40.649 & N/D & N/D & N/D & N/D & N/D & 157.827 & N/D & 0.534 & N/D & N/D & N/D & N/D & N/D \\
\hline 8.5 .2013 & 15.5.2013 & 8.997 & N/D & N/D & N/D & N/D & N/D & 36.039 & N/D & N/D & N/D & N/D & N/D & N/D & N/D \\
\hline 22.5.2013 & 29.5 & 17.040 & N/D & N/D & N/D & N/D & N/D & 3.240 & N/D & 0.442 & N/D & N/D & N/D & N/D & N/D \\
\hline 5.6 .2013 & 12.6.2013 & 2.466 & N/D & N/D & N/D & N/D & N/D & 3.791 & N/D & N/D & N/D & N/D & N/D & N/D & N/D \\
\hline 19.6 .2013 & 26.6.2013 & 10.626 & N/D & N/D & N/D & N/D & N/D & 14.671 & N/D & N/D & N/D & N/D & N/D & N/D & N/D \\
\hline 3.7 .2 & 10.7. & 1.199 & N/D & 0.206 & N/D & N/D & N/D & 17.982 & N/D & N/D & N/D & N/D & N/D & N/D & N/D \\
\hline 17.7.2013 & 24.7.2013 & N/D & N/D & N/D & N/D & N/D & N/D & 17.043 & N/D & N/D & N/D & N/D & N/D & N/D & 0.019 \\
\hline 31.7 .2013 & 7.8.2013 & N/D & N/D & N/D & N/D & N/D & N/D & 2.746 & N/D & N/D & N/D & N/D & N/D & N/D & N/D \\
\hline 14.8 .2013 & 21.8 .2013 & 0.981 & N/D & N/D & N/D & N/D & N/D & 1.280 & N/D & 6.467 & N/D & N/D & N/D & N/D & N/D \\
\hline 28.8 .2013 & 4.9.2013 & 0.575 & N/D & 0.084 & N/D & N/D & N/D & 2.449 & N/D & 60.233 & N/D & N/D & N/D & N/D & N/D \\
\hline 11.9.2013 & 18.9. & N/D & N/D & 0.023 & N/D & N/D & N/D & 1.831 & N/D & 5.664 & N/D & N/D & N/D & N/D & N/D \\
\hline 25.9 .2013 & 2.10 .2013 & 0.984 & N/D & N/D & N/D & N/D & N/D & 10.126 & N/D & 3.957 & N/D & N/D & N/D & N/D & $\mathrm{N} / \mathrm{D}$ \\
\hline 9.10 .2013 & 16.10 .2013 & N/D & N/D & N/D & N/D & N/D & 0.427 & 36.491 & N/D & 0.898 & $\mathrm{~N} / \mathrm{D}$ & N/D & N/D & N/D & N/D \\
\hline 23.10 .2013 & 30.10 .2013 & 0.284 & N/D & 0.096 & N/D & N/D & 0.483 & 64.530 & N/D & 0.602 & N/D & N/D & N/D & N/D & N/D \\
\hline 6.11 .2013 & 13.11.2013 & N/D & N/D & N/D & N/D & N/D & N/D & 25.223 & N/D & N/D & N/D & N/D & N/D & N/D & N/D \\
\hline 20.11.2013 & 27.11 .2013 & N/D & N/D & N/D & N/D & N/D & N/D & 14.913 & N/D & N/D & N/D & N/D & N/D & N/D & N/D \\
\hline 4.12 .2013 & 11.12 .2013 & N/D & N/D & $\mathrm{N} / \mathrm{D}$ & N/D & N/D & N/D & 2.946 & N/D & N/D & N/D & N/D & N/D & N/D & $\mathrm{N} / \mathrm{D}$ \\
\hline 18.12.2013 & 25.12.2013 & N/D & N/D & N/D & N/D & N/D & N/D & 2.897 & N/D & N/D & N/D & N/D & N/D & N/D & N/D \\
\hline
\end{tabular}


Table S12 (continued): Concentration of individual CUPs (in pg. $\mathrm{m}^{-3}$ ) in the gaseous phase at the background site.

\begin{tabular}{|c|c|c|c|c|c|c|c|c|c|c|c|c|c|c|}
\hline 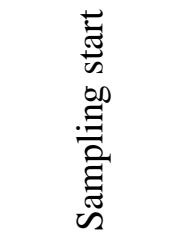 & 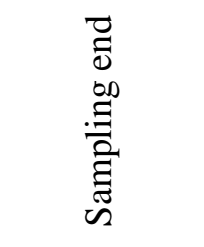 & 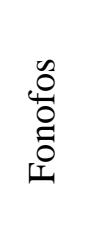 & 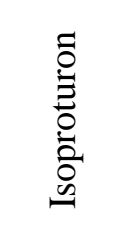 & 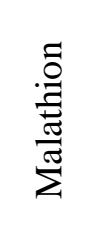 & 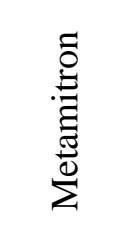 & 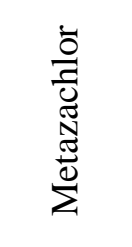 & 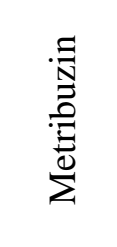 & 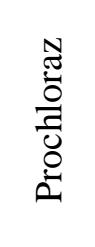 & 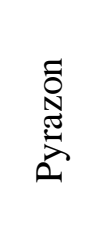 & 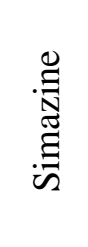 & $\begin{array}{l}\frac{\tilde{0}}{2} \\
\frac{0}{0} \\
\frac{\pi}{0} \\
\sum_{i=1}^{0} \\
i n\end{array}$ & $\frac{\tilde{O}}{\frac{\mathscr{C}}{2}}$ & 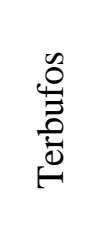 & 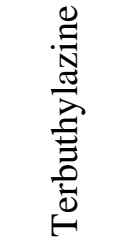 \\
\hline 4.1.2012 & 11.1.2012 & N/D & N/D & N/D & N/D & N/D & N/D & N/D & N/D & N/D & N/D & N/D & N/D & N/D \\
\hline 18.1.2012 & 25.1.2012 & N/D & N/D & N/D & 7.401 & N/D & N/D & N/D & N/D & N/D & N/D & N/D & N/D & N/D \\
\hline 1.2 .2012 & 8.2 .2012 & N/D & 0.039 & N/D & N/D & N/D & N/D & N/D & N/D & N/D & N/D & N/D & N/D & N/D \\
\hline 15.2 .2012 & 22.2 .2012 & N/D & N/D & N/D & N/D & N/D & N/D & N/D & N/D & N/D & N/D & N/D & N/D & $<\mathrm{LOQ}$ \\
\hline 29.2.2012 & 3.2012 & N/D & N/D & N/D & N/D & N/D & N/D & N/D & N/D & N/D & N/D & N/D & N/D & N/D \\
\hline 14.3 .2012 & 21.3.2012 & N/D & N/D & N/D & 2.674 & N/D & N/D & N/D & N/D & N/D & N/D & N/D & N/D & 0.040 \\
\hline 28.3.2012 & 4.4.2012 & N/D & N/D & N/D & N/D & N/D & N/D & N/D & N/D & N/D & N/D & N/D & N/D & N/D \\
\hline 11.4 .2012 & 18.4 .2012 & N/D & $<$ LOQ & N/D & N/D & 0.111 & N/D & N/D & N/D & N/D & 1.598 & N/D & N/D & 0.190 \\
\hline 25.4 .2012 & 2.5.2012 & N/D & 0.641 & $\mathrm{~N} / \mathrm{D}$ & N/D & 0.074 & N/D & N/D & N/D & N/D & 309.479 & N/D & N/D & 17.641 \\
\hline 9.5 .2012 & 16.5.2012 & N/D & 0.046 & N/D & N/D & 0.060 & 5.462 & N/D & N/D & N/D & 160.401 & N/D & N/D & 22.208 \\
\hline 23.5 .2012 & & N/D & & $\mathrm{N} / \mathrm{D}$ & N/D & & & N/D & N/D & N/D & & N/D & N/D & 10.310 \\
\hline 6.6.2012 & 13.6.2012 & N/D & N/D & $\mathrm{N} / \mathrm{D}$ & N/D & N/D & N/D & $\mathrm{N} / \mathrm{D}$ & N/D & N/D & 37.223 & N/D & N/D & 13.278 \\
\hline 20.6.2012 & 27.6.2012 & N/D & 0.136 & N/D & N/D & 0.299 & N/D & N/D & N/D & N/D & 47.040 & $<\mathrm{LOQ}$ & N/D & 25.588 \\
\hline 4.7.2012 & 11.7.2012 & N/D & 0.063 & N/D & $\mathrm{N} / \mathrm{D}$ & 0.183 & N/D & N/D & N/D & N/D & 11.830 & N/D & N/D & 9.971 \\
\hline 18.7.2012 & 25.7.2012 & N/D & N/D & N/D & N/D & 0.176 & N/D & N/D & N/D & N/D & 3.780 & N/D & N/D & 3.706 \\
\hline 1.8 .2012 & 8.8.2012 & $\mathrm{N} / \mathrm{D}$ & N/D & N/D & N/D & 0.306 & N/D & N/D & N/D & N/D & 1.338 & N/D & N/D & 1.873 \\
\hline 15.8 .2012 & 22.8 .2012 & N/D & N/D & $\mathrm{N} / \mathrm{D}$ & N/D & 50.614 & N/D & N/D & N/D & N/D & 0.370 & N/D & N/D & 0.367 \\
\hline 29.8.2012 & 5.9 .2012 & N/D & N/D & $\mathrm{N} / \mathrm{D}$ & N/D & 80.598 & 0.937 & N/D & N/D & N/D & 0.540 & N/D & $\mathrm{N} / \mathrm{D}$ & 0.710 \\
\hline 12.9 .2012 & 19.9.2012 & N/D & 0.106 & N/D & N/D & 15.246 & N/D & N/D & N/D & N/D & 0.571 & N/D & N/D & 1.106 \\
\hline 26.9.2012 & 3.10 .2012 & $\mathrm{~N} / \mathrm{D}$ & 0.044 & N/D & N/D & 3.855 & N/D & N/D & $\mathrm{N} / \mathrm{D}$ & N/D & 0.861 & N/D & N/D & 1.287 \\
\hline 10.10 .2012 & 17.10.2012 & N/D & 0.095 & N/D & N/D & 0.690 & N/D & N/D & N/D & N/D & 0.204 & N/D & N/D & 0.437 \\
\hline 24.10 .2012 & 31.10 .2012 & N/D & 0.406 & $\mathrm{~N} / \mathrm{D}$ & N/D & 0.480 & N/D & N/D & N/D & N/D & 0.116 & N/D & N/D & 0.286 \\
\hline 7.11.2012 & 14.11.2012 & N/D & 0.152 & N/D & 10.129 & 0.326 & N/D & N/D & N/D & N/D & 0.101 & N/D & N/D & 0.221 \\
\hline
\end{tabular}




\begin{tabular}{|c|c|c|c|c|c|c|c|c|c|c|c|c|c|c|}
\hline 22.11.2012 & 28.11.2012 & N/D & 0.471 & N/D & 14.706 & 0.347 & N/D & N/D & N/D & N/D & 0.195 & N/D & N/D & 0.261 \\
\hline 5.12 .2012 & 12.12 .2012 & $\mathrm{~N} / \mathrm{D}$ & 0.051 & N/D & N/D & 0.057 & N/D & N/D & N/D & N/D & N/D & N/D & N/D & N/D \\
\hline 19.12.2012 & 26.12.2012 & N/D & 0.033 & $\mathrm{~N} / \mathrm{D}$ & 16.470 & 0.081 & N/D & N/D & N/D & N/D & 0.060 & N/D & N/D & 0.068 \\
\hline 2.1.2013 & 9.1 .2013 & N/D & N/D & N/D & N/D & N/D & N/D & N/D & N/D & N/D & N/D & N/D & N/D & N/D \\
\hline 16.1 . & 23.1 .2 & N/D & N/D & N/D & N/D & N/D & N/D & N/D & N/D & $\mathrm{N} / \mathrm{D}$ & N/D & N/D & N/D & $<\mathrm{LOQ}$ \\
\hline 30.1 & 6.2 .2 & N/A & N/A & N/A & N/A & N/A & N/A & N/A & N/A & N/A & N/A & N/A & N/A & N/A \\
\hline 13.2 & 20.2 & N/D & N/D & $\mathrm{N} / \mathrm{D}$ & N/D & N/D & N/D & N/D & N/D & N/D & N/D & N/D & N/D & N/D \\
\hline 27.2 & 6.3 & J/D & N/D & $\mathrm{N} / \mathrm{D}$ & N/D & N/D & N/D & N/D & N/D & N/D & N/D & N/D & N/D & N/D \\
\hline 13.3 & 20.3 & N/D & //D & N/D & N/D & N/D & N/D & N/D & N/D & N/D & N/D & N/D & N/D & $\mathrm{N} / \mathrm{D}$ \\
\hline 27.3. & 3.4 .2013 & N/D & N/D & N/D & N/D & N/D & N/D & N/D & N/D & N/D & N/D & N/D & N/D & N/D \\
\hline 10.4 & 17.4 & N/D & N/D & N/D & N/D & N/D & N/D & N/D & N/D & $\mathrm{N} / \mathrm{D}$ & N/D & N/D & N/D & $<\mathrm{LOQ}$ \\
\hline 24.4 & 1.5 & $\mathrm{~N} / \mathrm{D}$ & 122.401 & N/D & N/D & 1.789 & N/D & N/D & N/D & N/D & 9.969 & N/D & N/D & 4.860 \\
\hline 8.5. & 15.5 & N/D & 7.684 & N/D & N/D & 0.764 & N/D & N/D & N/D & N/D & 69.358 & N/D & N/D & 15.345 \\
\hline 22.5 & 29.9 & N/D & 0.256 & N/D & $\mathrm{N} / \mathrm{D}$ & .132 & $\mathrm{~N} / \mathrm{D}$ & N/D & N/D & N/D & 196.914 & N/D & N/D & 14.570 \\
\hline 5.6 .2013 & 12.6 . & N/D & 0.313 & N/D & N/D & $<\mathrm{LOQ}$ & 0.738 & N/D & N/D & $\mathrm{N} / \mathrm{D}$ & 5.138 & N/D & N/D & 1.482 \\
\hline 19.6. & 26.6 & N/D & 1.616 & N/D & N/D & 0.272 & N/D & N/D & N/D & N/D & 20.498 & N/D & N/D & 33.821 \\
\hline 3.7 . & 10.7 & N/D & 0.447 & N/D & N/D & N/D & N/D & N/D & N/D & N/D & 22.346 & J/D & N/D & 10.232 \\
\hline 17.7 & 24.7 & N/D & 0.159 & 0.298 & N/D & N/D & N/D & N/D & N/D & N/D & 1.357 & N/D & N/D & 3.509 \\
\hline 31.7 & 7.8. & N/D & N/D & N/D & N/D & 0.696 & N/D & N/D & N/D & N/D & 0.145 & N/D & N/D & N/D \\
\hline 14.8. & 21.8 & N/D & N/D & J/D & 7.178 & 0.552 & N/D & N/D & N/D & N/D & 0.3 & N/D & N/D & 0.191 \\
\hline 28.8.2013 & 4.9 .20 & N/D & 0.061 & N/D & 1.845 & 262.115 & N/D & N/D & 0.801 & N/D & 1.386 & N/D & N/D & 0.706 \\
\hline 11.9 & 18.9 & N/D & & N/D & 0.238 & 28.069 & N/D & N/D & N/D & N/D & 0.3 & N/D & N/D & 0.247 \\
\hline 25.9 & 2.10 & N/D & 0.056 & N/D & 1.028 & 25.424 & N/D & N/D & N/D & N/D & 0.531 & N/D & N/D & 0.259 \\
\hline 9.10 .2013 & 16.10 .2013 & N/D & 0.475 & N/D & 1.072 & 12.096 & N/D & N/D & N/D & N/D & 0.449 & N/D & N/D & 0.235 \\
\hline 23.10.2013 & 30.10 .2013 & N/D & 0.309 & N/D & N/D & 7.480 & 0.691 & N/D & N/D & N/D & 0.307 & N/D & N/D & 0.402 \\
\hline 6.11 .2013 & 13.11.2013 & N/D & N/D & N/D & N/D & N/D & N/D & N/D & N/D & N/D & N/D & 0.207 & N/D & N/D \\
\hline 20.11.2013 & 27.11.2013 & N/D & N/D & N/D & N/D & 0.404 & 0.240 & N/D & N/D & N/D & 0.130 & N/D & N/D & 0.022 \\
\hline 4.12 .2013 & 11.12 .2013 & N/D & N/D & N/D & N/D & N/D & 1.199 & N/D & $\mathrm{N} / \mathrm{D}$ & N/D & N/D & N/D & N/D & N/D \\
\hline 18.12.2013 & 25.12.2013 & N/D & N/D & N/D & N/D & 0.467 & N/D & N/D & N/D & N/D & 0.061 & N/D & N/D & 0.022 \\
\hline
\end{tabular}


Table S13: Concentration of individual CUPs (in pg. $\mathrm{m}^{-3}$ ) in the particulate phase at the background site. N/D indicates compounds that were not detected and <LOQ indicates samples that were below the limit of quantification (in Table S7).

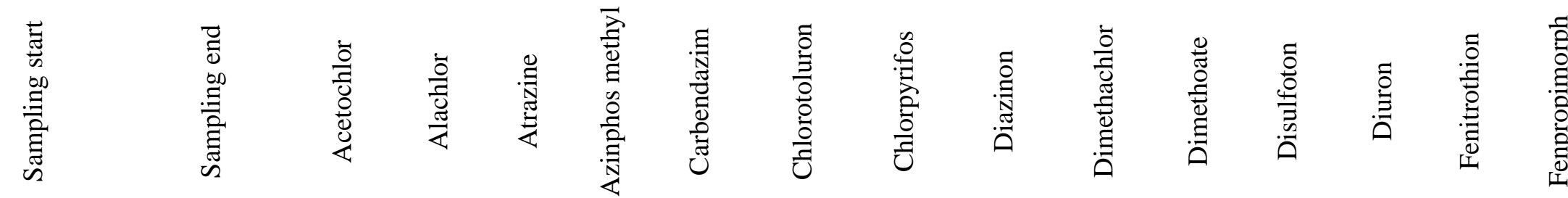

\begin{tabular}{|c|c|c|c|c|c|c|c|c|c|c|c|c|c|c|c|}
\hline 4.1 .2012 & 11.1.2012 & N/D & N/D & N/D & N/D & N/D & N/D & $<\mathrm{LOQ}$ & N/D & N/D & N/D & N/D & N/D & N/D & N/D \\
\hline 18.1.2012 & 25.1 .2012 & N/D & N/D & N/D & N/D & N/D & $<\mathrm{LOQ}$ & N/D & N/D & N/D & N/D & N/D & N/D & N/D & N/D \\
\hline 1.2 .2012 & 8.2.2012 & N/D & N/D & N/D & N/D & N/D & N/D & N/D & N/D & N/D & N/D & N/D & N/D & N/D & N/D \\
\hline 15.2 .2012 & 22.2.2012 & N/D & N/D & N/D & N/D & N/D & N/D & N/D & N/D & N/D & N/D & N/D & N/D & N/D & N/D \\
\hline 29.2.2012 & 7.3.2012 & 0.532 & N/D & N/D & N/D & N/D & N/D & N/D & N/D & N/D & N/D & N/D & N/D & N/D & N/D \\
\hline 14.3 .2012 & 21.3.2012 & 0.566 & N/D & N/D & N/D & N/D & 0.368 & N/D & N/D & N/D & N/D & N/D & N/D & N/D & N/D \\
\hline 28.3.2012 & 4.4.2012 & N/D & N/D & N/D & N/D & N/D & 0.505 & 9.428 & N/D & N/D & N/D & N/D & N/D & N/D & 0.160 \\
\hline 11.4 .2012 & 18.4 .2012 & N/D & N/D & N/D & N/D & N/D & 1.411 & 3.301 & N/D & N/D & N/D & N/D & N/D & N/D & 0.171 \\
\hline 25.4.2012 & 2.5.2012 & 1.712 & 0.442 & N/D & N/D & 1.974 & 3.693 & 0.451 & N/D & N/D & N/D & N/D & N/D & N/D & 0.745 \\
\hline 9.5 .2012 & 16.5.2012 & 23.247 & 0.819 & N/D & N/D & 2.618 & 0.655 & 0.280 & N/D & N/D & N/D & N/D & N/D & N/D & 73.802 \\
\hline 23.5.2012 & 30.5 .2012 & 0.528 & 0.279 & 0.487 & N/D & 1.983 & 0.135 & 0.117 & N/D & N/D & N/D & N/D & N/D & N/D & 10.129 \\
\hline 6.6 .2012 & 13.6.2012 & 0.577 & N/D & N/D & N/D & 0.252 & N/D & 0.760 & N/D & N/D & N/D & N/D & N/D & N/D & 5.561 \\
\hline 20.6.2012 & 27.6.2012 & N/D & N/D & N/D & N/D & N/D & N/D & 0.267 & N/D & N/D & 0.027 & N/D & N/D & N/D & 0.392 \\
\hline 4.7.2012 & 11.7 .2012 & N/D & N/D & N/D & N/D & N/D & N/D & N/D & N/D & N/D & N/D & N/D & 0.156 & N/D & 1.168 \\
\hline 18.7.2012 & 25.7.2012 & N/D & N/D & N/D & N/D & 0.355 & N/D & 0.250 & N/D & N/D & N/D & N/D & 0.265 & N/D & 0.467 \\
\hline 1.8.2012 & 8.8.2012 & N/D & N/D & N/D & N/D & N/D & N/D & N/D & N/D & N/D & N/D & N/D & 0.240 & N/D & 0.206 \\
\hline 15.8.2012 & 22.8.2012 & N/D & N/D & N/D & N/D & N/D & N/D & N/D & N/D & 1.851 & N/D & N/D & 0.205 & N/D & 0.151 \\
\hline 29.8.2012 & 5.9 .2012 & N/D & N/D & N/D & N/D & N/D & N/D & 0.143 & N/D & 0.519 & N/D & N/D & N/D & N/D & 1.506 \\
\hline 12.9 .2012 & 19.9.2012 & N/D & N/D & N/D & N/D & N/D & N/D & N/D & N/D & N/D & N/D & N/D & 0.136 & N/D & 0.026 \\
\hline 26.9.2012 & 3.10 .2012 & N/D & N/D & N/D & N/D & N/D & 2.434 & 0.166 & N/D & N/D & N/D & N/D & 0.211 & N/D & 0.013 \\
\hline 10.10.2012 & 17.10 .2012 & $\mathrm{~N} / \mathrm{D}$ & N/D & N/D & $\mathrm{N} / \mathrm{D}$ & N/D & 7.681 & 0.553 & N/D & N/D & N/D & N/D & 0.098 & N/D & N/D \\
\hline 24.10.2012 & 31.10 .2012 & N/D & N/D & N/D & N/D & N/D & 12.900 & 0.631 & $\mathrm{~N} / \mathrm{D}$ & N/D & N/D & $\mathrm{N} / \mathrm{D}$ & 0.351 & N/D & N/D \\
\hline 7.11 .2012 & 14.11.2012 & N/D & N/D & N/D & N/D & N/D & 2.728 & 0.398 & N/D & N/D & N/D & N/D & N/D & N/D & $<\mathrm{LOQ}$ \\
\hline 22.11.2012 & 28.11 .2012 & N/D & N/D & N/D & N/D & N/D & 0.802 & N/D & N/D & N/D & N/D & N/D & N/D & N/D & N/D \\
\hline 5.12 .2012 & 12.12 .2012 & N/D & N/D & N/D & N/D & N/D & N/D & N/D & N/D & N/D & N/D & N/D & N/D & N/D & N/D \\
\hline 19.12.2012 & 26.12 .2012 & N/D & N/D & N/D & N/D & N/D & 0.185 & N/D & N/D & N/D & N/D & N/D & N/D & N/D & $<\mathrm{LOQ}$ \\
\hline
\end{tabular}




\begin{tabular}{|c|c|c|c|c|c|c|c|c|c|c|c|c|c|c|c|}
\hline 2.1 .2013 & 9.1 .2013 & N/D & N/D & N/D & N/D & N/D & N/D & 0.121 & N/D & N/D & N/D & N/D & N/D & $\mathrm{N} / \mathrm{D}$ & $<\mathrm{LOQ}$ \\
\hline 16.1 .2013 & 23.1 .2013 & N/D & N/D & N/D & N/D & N/D & N/D & N/D & N/D & N/D & N/D & N/D & N/D & N/D & N/D \\
\hline 30.1 .2013 & 6.2 .2013 & N/D & N/D & N/D & N/D & N/D & N/D & N/D & N/D & N/D & N/D & N/D & N/D & N/D & $<\mathrm{LOQ}$ \\
\hline 13.2 .2013 & 20.2.2013 & N/D & N/D & N/D & $\mathrm{N} / \mathrm{D}$ & N/D & N/D & N/D & N/D & N/D & N/D & N/D & N/D & N/D & N/D \\
\hline 27.2.2013 & 6.3 .2013 & N/D & N/D & N/D & N/D & N/D & N/D & N/D & N/D & N/D & N/D & N/D & N/D & $\mathrm{N} / \mathrm{D}$ & N/D \\
\hline 13.3 .2013 & 20.3 .2013 & N/D & N/D & N/D & N/D & $\mathrm{N} / \mathrm{D}$ & N/D & N/D & N/D & N/D & N/D & N/D & 1.232 & N/D & $<\mathrm{LOQ}$ \\
\hline 27.3.2013 & 3.4 .2013 & N/D & N/D & N/D & N/D & N/D & N/D & N/D & N/D & N/D & N/D & N/D & N/D & N/D & N/D \\
\hline 10.4 .2013 & 17.4 .2013 & 1.155 & N/D & N/D & N/D & N/D & 2.675 & 0.142 & N/D & N/D & N/D & N/D & 0.353 & N/D & N/D \\
\hline 24.4 .2013 & 1.5 .2013 & 11.854 & N/D & N/D & N/D & N/D & 6.063 & 1.512 & N/D & N/D & N/D & N/D & N/D & N/D & 0.544 \\
\hline 8.5.2013 & 15.5 .2013 & 5.123 & N/D & N/D & N/D & 1.281 & 0.324 & 0.231 & N/D & N/D & N/D & N/D & N/D & N/D & 4.253 \\
\hline 22.5 .2013 & 29.5.2013 & 2.810 & N/D & N/D & N/D & 1.353 & 0.428 & 0.166 & N/D & N/D & N/D & N/D & N/D & N/D & 29.631 \\
\hline 5.6 .2013 & 12.6.2013 & N/D & N/D & N/D & N/D & 5.937 & 0.136 & N/D & N/D & N/D & N/D & N/D & N/D & N/D & 48.356 \\
\hline 19.6.2013 & 26.6.2013 & N/D & N/D & N/D & N/D & 1.529 & N/D & N/D & N/D & N/D & N/D & N/D & N/D & N/D & 5.386 \\
\hline 3.7 .2013 & 10.7.2013 & N/D & N/D & N/D & N/D & 5.965 & N/D & 0.117 & N/D & N/D & N/D & N/D & 0.080 & N/D & 19.138 \\
\hline 17.7.2013 & 24.7.2013 & N/D & N/D & 0.063 & N/D & 8.289 & $<\mathrm{LOQ}$ & 0.416 & $<\mathrm{LOQ}$ & N/D & N/D & N/D & 0.145 & N/D & 9.612 \\
\hline 31.7.2013 & 7.8.2013 & N/D & N/D & 0.247 & $\mathrm{~N} / \mathrm{D}$ & 2.161 & $\mathrm{~N} / \mathrm{D}$ & 0.423 & N/D & 0.485 & N/D & N/D & 0.746 & N/D & 3.217 \\
\hline 14.8 .2013 & 21.8 .2013 & $\mathrm{~N} / \mathrm{D}$ & N/D & N/D & $\mathrm{N} / \mathrm{D}$ & 1.286 & N/D & $<\mathrm{LOQ}$ & $\mathrm{N} / \mathrm{D}$ & 9.357 & N/D & $\mathrm{N} / \mathrm{D}$ & 0.117 & $\mathrm{~N} / \mathrm{D}$ & 0.936 \\
\hline 28.8 .2013 & 4.9 .2013 & N/D & N/D & 0.082 & $\mathrm{~N} / \mathrm{D}$ & 2.528 & N/D & N/D & N/D & 1.290 & $\mathrm{~N} / \mathrm{D}$ & N/D & $<\mathrm{LOQ}$ & N/D & 15.632 \\
\hline 11.9 .2013 & 18.9 .2013 & N/D & N/D & N/D & $\mathrm{N} / \mathrm{D}$ & 5.172 & N/D & N/D & N/D & 0.611 & 0.081 & N/D & $<\mathrm{LOQ}$ & N/D & 1.118 \\
\hline 25.9 .2013 & 2.10 .2013 & N/D & N/D & 0.097 & N/D & 12.525 & 2.114 & $\mathrm{~N} / \mathrm{D}$ & N/D & 0.230 & N/D & N/D & 0.074 & N/D & 0.469 \\
\hline 9.10 .2013 & 16.10 .2013 & N/D & N/D & N/D & N/D & 11.419 & 2.513 & 1.646 & N/D & N/D & N/D & $\mathrm{N} / \mathrm{D}$ & N/D & N/D & 1.291 \\
\hline 23.10 .2013 & 30.10 & N/D & N/D & N/D & N/D & 8.242 & 24.665 & 0.112 & $<\mathrm{LOQ}$ & N/D & N/D & 2.216 & 0.098 & N/D & 0.922 \\
\hline 6.11 .2 & 13.11 & N/D & N/D & 0.140 & N/D & 7.554 & 5.739 & 0.131 & N/D & 0.335 & N/D & N/D & 0.072 & N/D & 0.358 \\
\hline 20.11 . & 27.11 .2013 & N/D & N/D & 0.041 & N/D & 8.460 & 1.106 & N/D & N/D & 0.123 & N/D & N/D & $<\mathrm{LOQ}$ & N/D & 0.279 \\
\hline 4.12 .2013 & 11.12 .2013 & N/D & N/D & 0.038 & N/D & 1.388 & 0.252 & 0.156 & N/D & $<\mathrm{LOQ}$ & N/D & N/D & N/D & N/D & 0.542 \\
\hline 18.12 .2013 & 25.12 .2013 & N/D & N/D & N/D & N/D & 12.107 & 0.266 & 0.250 & $<\mathrm{LOQ}$ & N/D & N/D & N/D & N/D & N/D & 0.698 \\
\hline
\end{tabular}


Table S13 (continued): Concentration of individual CUPs (in pg. $\mathrm{m}^{-3}$ ) in the particulate phase at the background site.

\begin{tabular}{|c|c|c|c|c|c|c|c|c|c|c|c|c|c|}
\hline 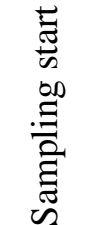 & 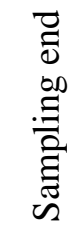 & $\begin{array}{l}00 \\
0 \\
0 \\
0 \\
0 \\
0\end{array}$ & $\begin{array}{l}0 \\
0 \\
0 \\
0 \\
0 \\
0 \\
0.0\end{array}$ & 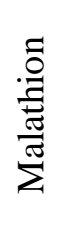 & 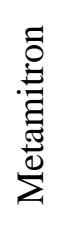 & 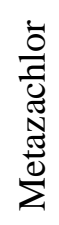 & 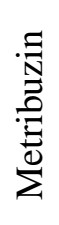 & 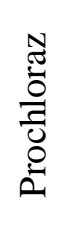 & 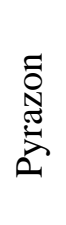 & $\begin{array}{l}\text { : } \\
\text { : } \\
\text { : }\end{array}$ & $\begin{array}{l}\frac{\tilde{0}}{0} \\
\frac{0}{0} \\
\frac{\pi}{0} \\
\sum_{i}^{0} \\
\text { is }\end{array}$ & $\frac{0}{0}$ & 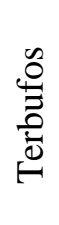 \\
\hline
\end{tabular}

\begin{tabular}{|c|c|c|c|c|c|c|c|c|c|c|c|c|c|c|}
\hline 4.1 .2012 & 11.1 .2012 & $\mathrm{~N} / \mathrm{D}$ & 0.213 & N/D & N/D & 0.371 & $\mathrm{~N} / \mathrm{D}$ & $\mathrm{N} / \mathrm{D}$ & N/D & $\mathrm{N} / \mathrm{D}$ & 0.146 & N/D & N/D & 0.155 \\
\hline 18.1 .2012 & 25.1 .2012 & N/D & 0.094 & N/D & N/D & $\mathrm{N} / \mathrm{D}$ & $\mathrm{N} / \mathrm{D}$ & N/D & $\mathrm{N} / \mathrm{D}$ & N/D & $\mathrm{N} / \mathrm{D}$ & N/D & N/D & N/D \\
\hline 1.2 .2012 & 8.2.2012 & N/D & N/D & N/D & N/D & N/D & $\mathrm{N} / \mathrm{D}$ & N/D & N/D & N/D & N/D & N/D & N/D & N/D \\
\hline 15.2.2012 & 22.2.2012 & N/D & 0.061 & N/D & N/D & N/D & $\mathrm{N} / \mathrm{D}$ & N/D & N/D & N/D & N/D & N/D & N/D & N/D \\
\hline 29.2.2012 & 7.3.2012 & N/D & 0.224 & N/D & N/D & 0.088 & N/D & N/D & N/D & N/D & N/D & N/D & N/D & N/D \\
\hline 14.3 .2012 & 21.3 .2012 & N/D & 1.836 & $\mathrm{~N} / \mathrm{D}$ & N/D & 0.135 & N/D & 0.354 & N/D & $\mathrm{N} / \mathrm{D}$ & N/D & N/D & N/D & 0.100 \\
\hline 28.3.2012 & 4.4.2012 & N/D & 5.612 & N/D & N/D & 0.198 & N/D & 0.212 & N/D & N/D & N/D & N/D & N/D & 0.258 \\
\hline 11.4 .2012 & 18.4.2012 & N/D & 5.494 & $\mathrm{~N} / \mathrm{D}$ & N/D & 0.087 & $\mathrm{~N} / \mathrm{D}$ & 0.132 & N/D & $\mathrm{N} / \mathrm{D}$ & 0.158 & N/D & $\mathrm{N} / \mathrm{D}$ & 0.260 \\
\hline 25.4.2012 & 2.5 .2012 & N/D & 20.241 & N/D & 3.784 & 0.262 & N/D & 1.493 & 1.049 & N/D & 19.603 & N/D & N/D & 10.235 \\
\hline 9.5 .2012 & 16.5 .2012 & N/D & 5.033 & N/D & N/D & 0.876 & N/D & 1.659 & N/D & N/D & 90.955 & N/D & N/D & 31.597 \\
\hline 23.5 .2012 & 30.5 .2012 & N/D & 0.938 & $\mathrm{~N} / \mathrm{D}$ & 2.357 & 0.252 & N/D & 0.446 & 0.897 & $\mathrm{~N} / \mathrm{D}$ & 4.962 & N/D & N/D & 3.761 \\
\hline 6.6 .2012 & 13.6.2012 & N/D & 0.161 & N/D & N/D & 0.071 & N/D & 0.169 & N/D & N/D & 5.515 & N/D & N/D & 11.394 \\
\hline 20.6 .2012 & 27.6.2012 & N/D & 0.038 & N/D & N/D & $<\mathrm{LOQ}$ & N/D & 0.128 & N/D & N/D & 0.103 & $\mathrm{~N} / \mathrm{D}$ & N/D & 0.136 \\
\hline 4.7.2012 & 11.7.2012 & N/D & $<\mathrm{LOQ}$ & N/D & N/D & N/D & $\mathrm{N} / \mathrm{D}$ & N/D & N/D & $\mathrm{N} / \mathrm{D}$ & 0.138 & N/D & N/D & 0.704 \\
\hline 18.7.2012 & 25.7.2012 & N/D & $<\mathrm{LOQ}$ & N/D & N/D & N/D & N/D & N/D & N/D & N/D & 0.156 & N/D & N/D & 0.483 \\
\hline 1.8.2012 & 8.8.2012 & N/D & N/D & N/D & N/D & 0.919 & N/D & N/D & N/D & N/D & 0.157 & N/D & N/D & 0.206 \\
\hline 15.8.2012 & 22.8.2012 & $\mathrm{N} / \mathrm{D}$ & $<\mathrm{LOQ}$ & N/D & N/D & 30.166 & $\mathrm{~N} / \mathrm{D}$ & 0.055 & N/D & $\mathrm{N} / \mathrm{D}$ & $<\mathrm{LOQ}$ & N/D & N/D & 0.176 \\
\hline 29.8.2012 & 5.9 .2012 & N/D & N/D & N/D & N/D & 6.894 & N/D & 0.098 & N/D & N/D & N/D & N/D & N/D & 0.245 \\
\hline 12.9.2012 & 19.9.2012 & N/D & 0.051 & N/D & N/D & 2.159 & N/D & 0.136 & N/D & $\mathrm{N} / \mathrm{D}$ & N/D & N/D & N/D & 0.138 \\
\hline 26.9.2012 & 3.10 .2012 & N/D & 11.564 & N/D & N/D & 3.787 & N/D & 0.809 & N/D & N/D & 0.092 & N/D & N/D & 0.205 \\
\hline 10.10 .2012 & 17.10 .2012 & N/D & 27.769 & N/D & N/D & 0.634 & N/D & 0.282 & N/D & N/D & N/D & N/D & N/D & 0.212 \\
\hline 24.10 .2012 & 31.10 .2012 & N/D & 56.175 & N/D & N/D & 0.302 & N/D & 0.104 & $\mathrm{~N} / \mathrm{D}$ & N/D & N/D & N/D & N/D & 0.000 \\
\hline 7.11 .2012 & 14.11 .2012 & N/D & 2.817 & $\mathrm{~N} / \mathrm{D}$ & N/D & 0.140 & $\mathrm{~N} / \mathrm{D}$ & N/D & N/D & $\mathrm{N} / \mathrm{D}$ & N/D & N/D & N/D & 0.190 \\
\hline
\end{tabular}




\begin{tabular}{|c|c|c|c|c|c|c|c|c|c|c|c|c|c|c|}
\hline 22.11.2012 & 28.11.2012 & N/D & 4.183 & N/D & N/D & 0.271 & N/D & N/D & N/D & N/D & $\mathrm{N} / \mathrm{D}$ & N/D & N/D & N/D \\
\hline 5.12 .2012 & 12.12.2012 & N/D & 0.226 & N/D & N/D & N/D & N/D & N/D & N/D & N/D & N/D & N/D & N/D & N/D \\
\hline 19.12.2012 & 26.12.2012 & N/D & 0.410 & N/D & N/D & N/D & N/D & N/D & N/D & N/D & N/D & N/D & N/D & N/D \\
\hline 2.1 .2013 & 9.1 .2013 & N/D & 0.323 & N/D & N/D & 0.306 & N/D & N/D & N/D & N/D & $\mathrm{N} / \mathrm{D}$ & N/D & N/D & 0.112 \\
\hline 16.1 .2013 & 23.1.2013 & N/D & N/D & N/D & N/D & N/D & N/D & N/D & N/D & N/D & $\mathrm{N} / \mathrm{D}$ & N/D & N/D & N/D \\
\hline 30.1 .2013 & 6.2 .2013 & N/D & 0.156 & N/D & N/D & 0.368 & N/D & N/D & N/D & N/D & N/D & N/D & N/D & N/D \\
\hline 13.2.2013 & 20.2.2013 & N/D & N/D & N/D & N/D & N/D & N/D & N/D & N/D & N/D & N/D & N/D & N/D & N/D \\
\hline 27.2 & 6.3 .2 & N/D & 0.071 & N/D & N/D & 0.158 & N/D & N/D & N/D & N/D & N/D & N/D & N/D & N/D \\
\hline 13.3 .2013 & 20.3.2013 & N/D & N/D & N/D & N/D & N/D & N/D & N/D & N/D & N/D & N/D & N/D & N/D & N/D \\
\hline 27.3.2013 & 3.4 .2013 & N/D & 0.048 & N/D & N/D & N/D & N/D & N/D & N/D & N/D & N/D & N/D & N/D & N/D \\
\hline 10.4 .2013 & 17.4 .2013 & N/D & 4.653 & N/D & N/D & 0.888 & N/D & 0.147 & N/D & N/D & 0.877 & N/D & N/D & 0.490 \\
\hline 24.4 .2013 & 1.5.2013 & N/D & 290.673 & N/D & N/D & 0.806 & N/D & 1.637 & N/D & N/D & 3.520 & N/D & N/D & 6.813 \\
\hline 8.5 .2 & 15.5 & N/D & 13.804 & N/D & 6.414 & 0.069 & N/D & 0.258 & 0.415 & N/D & 1.927 & N/D & N/D & 5.734 \\
\hline 22.5 & & N/D & & N/D & N/D & 0.258 & & & N/D & N/D & 41.694 & D & N/D & \\
\hline 5.6.2013 & 12.6.2013 & N/D & 1.206 & N/D & N/D & 0.169 & N/D & 1.948 & N/D & N/D & 5.161 & N/D & N/D & 22.492 \\
\hline 19.6.2013 & 26.6.2013 & N/D & 0.233 & N/D & N/D & N/D & N/D & 0.119 & 1.090 & N/D & 0.132 & N/D & N/D & 0.526 \\
\hline 3.7 .2013 & 10.7.2013 & N/D & 0.265 & N/D & N/D & 0.158 & N/D & 0.872 & N/D & N/D & 0.482 & N/D & N/D & 2.288 \\
\hline 17.7.2013 & 24.7.2013 & N/D & 0.209 & N/D & N/D & 0.154 & N/D & 0.355 & 2.254 & 0.087 & 0.198 & N/D & N/D & 0.750 \\
\hline 31.7 . & 7.8.2013 & 8.028 & 1.305 & N/D & N/D & 0.332 & N/D & 0.251 & 1.023 & N/D & 2.659 & N/D & N/D & 7.352 \\
\hline 14.8.2013 & 21.8.2013 & 1.081 & 0.420 & N/D & N/D & 275.496 & N/D & 0.045 & 0.371 & N/D & 1.158 & N/D & N/D & 1.198 \\
\hline 28.8.2013 & 4.9.2013 & 0.650 & 0.163 & N/D & N/D & 82.106 & N/D & N/D & 0.280 & N/D & $<\mathrm{LOQ}$ & N/D & N/D & 0.165 \\
\hline 11.9 .2013 & 18.9.2013 & N/D & 0.259 & N/D & N/D & 123.587 & N/D & 0.264 & N/D & N/D & 0.295 & N/D & N/D & 0.254 \\
\hline 25.9 .2013 & 2.10 .2013 & N/D & 2.451 & $<\mathrm{LOQ}$ & N/D & 13.128 & N/D & 1.298 & N/D & N/D & $<\mathrm{LOQ}$ & N/D & N/D & 0.101 \\
\hline 9.10 .2013 & 16.10 .2013 & N/D & 5.894 & N/D & $\mathrm{N} / \mathrm{D}$ & 5.457 & 1.825 & 1.131 & N/D & N/D & $<\mathrm{LOQ}$ & 0.076 & N/D & 0.074 \\
\hline 23.10 .2013 & 30.10 .2013 & N/D & 33.710 & 0.132 & N/D & 30.236 & 1.795 & 0.126 & N/D & N/D & 0.171 & 0.105 & N/D & 0.259 \\
\hline 6.11 .2013 & 13.11 .2013 & N/D & 3.862 & N/D & N/D & 7.474 & N/D & N/D & N/D & N/D & 0.253 & N/D & N/D & 0.335 \\
\hline 20.11.2013 & 27.11.2013 & N/D & 1.529 & $\mathrm{~N} / \mathrm{D}$ & N/D & 1.618 & N/D & N/D & N/D & N/D & 0.070 & N/D & 0.614 & 0.036 \\
\hline 4.12 .2013 & 11.12.2013 & N/D & 0.226 & N/D & N/D & 0.567 & N/D & 0.030 & N/D & N/D & 0.061 & N/D & N/D & 0.027 \\
\hline 18.12.2013 & 25.12.2013 & N/D & 0.279 & N/D & N/D & 0.977 & N/D & N/D & N/D & N/D & $<\mathrm{LOQ}$ & N/D & $\mathrm{N} / \mathrm{D}$ & 0.049 \\
\hline
\end{tabular}


Table S14: Concentrations of individual OCPs (in pg. $\mathrm{m}^{-3}$ ) in the particulate phase. <LOQ indicates samples that were below the limit of quantification (in Table S7).

\begin{tabular}{|c|c|c|c|c|c|c|c|c|c|c|}
\hline Sampling time & $\alpha-\mathrm{HCH}$ & $\beta-\mathrm{HCH}$ & $\gamma-\mathrm{HCH}$ & $\delta-\mathrm{HCH}$ & $o, p^{\prime}-\mathrm{DDE}$ & $p, p^{\prime}-\mathrm{DDE}$ & $o, p^{\prime}-\mathrm{DDD}$ & $p, p^{\prime}$-DDD & $o, p^{\prime}$-DDT & $p, p^{\prime}-\mathrm{DDT}$ \\
\hline $11-18 / 01 / 12$ & $<\mathrm{LOQ}$ & 0.011 & $<L O Q$ & 0.008 & 0.007 & 0.169 & 0.068 & 0.096 & 0.040 & 0.149 \\
\hline $25 / 01-01 / 02 / 12$ & 0.031 & 0.030 & 0.025 & 0.029 & 0.051 & 0.900 & 0.109 & 0.399 & 0.099 & 0.288 \\
\hline $08-15 / 02 / 12$ & 0.012 & 0.018 & 0.018 & 0.020 & 0.054 & 0.526 & 0.076 & 0.345 & 0.114 & 0.462 \\
\hline $22-29 / 02 / 12$ & $<\mathrm{LOQ}$ & 0.020 & 0.000 & 0.027 & 0.007 & 0.209 & 0.047 & 0.098 & 0.037 & 0.106 \\
\hline $07-14 / 03 / 12$ & 0.022 & 0.020 & 0.042 & 0.025 & 0.011 & 0.388 & 0.052 & 0.141 & 0.074 & 0.189 \\
\hline $21-28 / 03 / 12$ & 0.027 & 0.020 & 0.043 & 0.064 & 0.016 & 0.583 & 0.063 & 0.250 & 0.113 & 0.317 \\
\hline 04-11/04/12 & 0.007 & 0.012 & 0.013 & 0.065 & 0.014 & 0.306 & 0.035 & 0.123 & 0.051 & 0.143 \\
\hline $18-25 / 04 / 12$ & $<\mathrm{LOQ}$ & 0.008 & $<\mathrm{LOQ}$ & 0.000 & 0.004 & 0.190 & 0.022 & 0.070 & 0.034 & 0.098 \\
\hline $02-09 / 05 / 12$ & 0.016 & 0.074 & 0.019 & 0.035 & 0.002 & 0.133 & 0.029 & 0.041 & 0.022 & 0.052 \\
\hline $16-23 / 05 / 12$ & 0.005 & 0.005 & 0.014 & 0.008 & 0.004 & 0.086 & 0.015 & 0.031 & 0.021 & 0.049 \\
\hline $30 / 05-06 / 06 / 12$ & 0.016 & 0.011 & $<\mathrm{LOQ}$ & 0.004 & 0.002 & 0.067 & 0.023 & 0.021 & 0.022 & 0.044 \\
\hline $13-20 / 06 / 12$ & 0.011 & $<\mathrm{LOQ}$ & 0.014 & $<\mathrm{LOQ}$ & $<\mathrm{LOQ}$ & 0.057 & 0.005 & 0.006 & 0.011 & 0.042 \\
\hline 27/06-04/07/12 & 0.008 & $<\mathrm{LOQ}$ & 0.014 & $<\mathrm{LOQ}$ & $<\mathrm{LOQ}$ & 0.048 & $<\mathrm{LOQ}$ & 0.006 & 0.010 & 0.036 \\
\hline 11-18/07/12 & 0.004 & $<\mathrm{LOQ}$ & 0.010 & $<\mathrm{LOQ}$ & $<\mathrm{LOQ}$ & 0.042 & $<\mathrm{LOQ}$ & 0.005 & 0.009 & 0.040 \\
\hline 25/07-01/08/12 & 0.003 & $<\mathrm{LOQ}$ & 0.007 & $<\mathrm{LOQ}$ & $<\mathrm{LOQ}$ & 0.060 & $<\mathrm{LOQ}$ & 0.004 & 0.006 & 0.023 \\
\hline 08-15/08/12 & 0.003 & $<\mathrm{LOQ}$ & 0.006 & $<\mathrm{LOQ}$ & $<\mathrm{LOQ}$ & 0.057 & $<\mathrm{LOQ}$ & 0.007 & 0.014 & 0.048 \\
\hline $22-29 / 08 / 12$ & 0.003 & $<\mathrm{LOQ}$ & 0.005 & $<\mathrm{LOQ}$ & $<\mathrm{LOQ}$ & 0.035 & $<\mathrm{LOQ}$ & 0.004 & 0.005 & 0.018 \\
\hline $05-12 / 09 / 12$ & 0.004 & $<\mathrm{LOQ}$ & 0.010 & $<\mathrm{LOQ}$ & $<\mathrm{LOQ}$ & 0.079 & $<\mathrm{LOQ}$ & 0.007 & 0.015 & 0.046 \\
\hline $19-26 / 09 / 12$ & 0.004 & $<\mathrm{LOQ}$ & 0.010 & $<\mathrm{LOQ}$ & $<\mathrm{LOQ}$ & 0.158 & $<\mathrm{LOQ}$ & 0.012 & 0.027 & 0.100 \\
\hline $03-10 / 10 / 12$ & 0.004 & $<\mathrm{LOQ}$ & 0.011 & $<\mathrm{LOQ}$ & $<\mathrm{LOQ}$ & 0.271 & $<\mathrm{LOQ}$ & 0.027 & 0.054 & 0.204 \\
\hline $17-24 / 10 / 12$ & 0.009 & 0.007 & 0.016 & $<\mathrm{LOQ}$ & $<\mathrm{LOQ}$ & 0.397 & $<\mathrm{LOQ}$ & 0.034 & 0.064 & 0.238 \\
\hline $31 / 11-07 / 11 / 12$ & 0.006 & 0.006 & 0.010 & $<\mathrm{LOQ}$ & $<\mathrm{LOQ}$ & 0.273 & $<\mathrm{LOQ}$ & 0.026 & 0.044 & 0.191 \\
\hline $14-21 / 11 / 12$ & 0.008 & 0.014 & 0.013 & $<\mathrm{LOQ}$ & $<\mathrm{LOQ}$ & 0.635 & $<\mathrm{LOQ}$ & 0.044 & 0.097 & 0.356 \\
\hline 28/11-05/12/12 & 0.006 & 0.004 & 0.016 & $<\mathrm{LOQ}$ & $<\mathrm{LOQ}$ & 0.501 & 0.014 & 0.076 & 0.093 & 0.419 \\
\hline $12-19 / 12 / 12$ & 0.010 & $<\mathrm{LOQ}$ & 0.024 & $<$ LOQ & $<\mathrm{LOQ}$ & 0.466 & $<\mathrm{LOQ}$ & 0.076 & 0.102 & 0.390 \\
\hline $6 / 12 / 12-02 / 01 / 13$ & 0.005 & $<\mathrm{LOQ}$ & 0.018 & $<\mathrm{LOQ}$ & $<\mathrm{LOQ}$ & 0.437 & 0.012 & 0.057 & 0.083 & 0.353 \\
\hline 09-16/01/13 & $<\mathrm{LOQ}$ & $<\mathrm{LOQ}$ & $<\mathrm{LOQ}$ & $<\mathrm{LOQ}$ & $<$ LOQ & 0.410 & $<\mathrm{LOQ}$ & $<\mathrm{LOQ}$ & $<\mathrm{LOQ}$ & 0.303 \\
\hline $23-30 / 01 / 13$ & $<\mathrm{LOQ}$ & $<\mathrm{LOQ}$ & $<\mathrm{LOQ}$ & $<\mathrm{LOQ}$ & $<\mathrm{LOQ}$ & 0.499 & $<\mathrm{LOQ}$ & $<\mathrm{LOQ}$ & $<\mathrm{LOQ}$ & 0.504 \\
\hline $06-13 / 02 / 13$ & $<\mathrm{LOQ}$ & $<\mathrm{LOQ}$ & $<\mathrm{LOQ}$ & $<\mathrm{LOQ}$ & $<\mathrm{LOQ}$ & 0.472 & $<\mathrm{LOQ}$ & $<\mathrm{LOQ}$ & $<\mathrm{LOQ}$ & 0.339 \\
\hline $20-27 / 02 / 13$ & $<\mathrm{LOQ}$ & $<\mathrm{LOQ}$ & $<\mathrm{LOQ}$ & $<\mathrm{LOQ}$ & $<\mathrm{LOQ}$ & 0.656 & $<\mathrm{LOQ}$ & $<\mathrm{LOQ}$ & $<\mathrm{LOQ}$ & 0.348 \\
\hline $06-13 / 03 / 13$ & $<\mathrm{LOQ}$ & $<\mathrm{LOQ}$ & $<\mathrm{LOQ}$ & $<\mathrm{LOQ}$ & $<\mathrm{LOQ}$ & 0.963 & $<\mathrm{LOQ}$ & $<\mathrm{LOQ}$ & $<\mathrm{LOQ}$ & 0.444 \\
\hline $20-27 / 03 / 13$ & $<\mathrm{LOQ}$ & $<\mathrm{LOQ}$ & $<\mathrm{LOQ}$ & $<\mathrm{LOQ}$ & $<\mathrm{LOQ}$ & 0.370 & $<\mathrm{LOQ}$ & $<\mathrm{LOQ}$ & $<\mathrm{LOQ}$ & 0.298 \\
\hline $03-10 / 04 / 13$ & $<\mathrm{LOQ}$ & $<\mathrm{LOQ}$ & $<\mathrm{LOQ}$ & $<\mathrm{LOQ}$ & $<\mathrm{LOQ}$ & 0.330 & $<\mathrm{LOQ}$ & $<\mathrm{LOQ}$ & $<\mathrm{LOQ}$ & $<\mathrm{LOQ}$ \\
\hline $17-24 / 04 / 13$ & $<\mathrm{LOQ}$ & $<\mathrm{LOQ}$ & $<\mathrm{LOQ}$ & $<\mathrm{LOQ}$ & $<\mathrm{LOQ}$ & 0.243 & $<\mathrm{LOQ}$ & $<\mathrm{LOQ}$ & $<\mathrm{LOQ}$ & 0.169 \\
\hline $01-08 / 05 / 13$ & $<\mathrm{LOQ}$ & $<\mathrm{LOQ}$ & $<\mathrm{LOQ}$ & $<\mathrm{LOQ}$ & $<\mathrm{LOQ}$ & 0.162 & $<\mathrm{LOQ}$ & $<\mathrm{LOQ}$ & $<\mathrm{LOQ}$ & $<\mathrm{LOQ}$ \\
\hline $15-22 / 05 / 13$ & $<\mathrm{LOQ}$ & $<\mathrm{LOQ}$ & $<\mathrm{LOQ}$ & $<\mathrm{LOQ}$ & $<\mathrm{LOQ}$ & 0.111 & $<\mathrm{LOQ}$ & $<\mathrm{LOQ}$ & $<\mathrm{LOQ}$ & $<\mathrm{LOQ}$ \\
\hline $29 / 05-05 / 06 / 13$ & $<\mathrm{LOQ}$ & $<\mathrm{LOQ}$ & $<\mathrm{LOQ}$ & $<\mathrm{LOQ}$ & $<\mathrm{LOQ}$ & 0.072 & $<\mathrm{LOQ}$ & $<\mathrm{LOQ}$ & $<\mathrm{LOQ}$ & $<\mathrm{LOQ}$ \\
\hline $12-19 / 06 / 13$ & $<\mathrm{LOQ}$ & $<\mathrm{LOQ}$ & $<\mathrm{LOQ}$ & $<\mathrm{LOQ}$ & $<\mathrm{LOQ}$ & 0.066 & $<\mathrm{LOQ}$ & $<\mathrm{LOQ}$ & $<\mathrm{LOQ}$ & $<\mathrm{LOQ}$ \\
\hline 26/06-03/07/13 & $<\mathrm{LOQ}$ & $<\mathrm{LOQ}$ & $<\mathrm{LOQ}$ & $<\mathrm{LOQ}$ & $<\mathrm{LOQ}$ & $<\mathrm{LOQ}$ & $<\mathrm{LOQ}$ & $<\mathrm{LOQ}$ & $<\mathrm{LOQ}$ & $<\mathrm{LOQ}$ \\
\hline 10-17/07/13 & $<\mathrm{LOQ}$ & $<\mathrm{LOQ}$ & $<\mathrm{LOQ}$ & $<\mathrm{LOQ}$ & $<\mathrm{LOQ}$ & $<\mathrm{LOQ}$ & $<\mathrm{LOQ}$ & $<\mathrm{LOQ}$ & $<\mathrm{LOQ}$ & $<\mathrm{LOQ}$ \\
\hline 24-31/07/13 & $<\mathrm{LOQ}$ & $<\mathrm{LOQ}$ & $<\mathrm{LOQ}$ & $<\mathrm{LOQ}$ & $<\mathrm{LOQ}$ & $<\mathrm{LOQ}$ & $<\mathrm{LOQ}$ & $<\mathrm{LOQ}$ & $<\mathrm{LOQ}$ & $<\mathrm{LOQ}$ \\
\hline $07-14 / 08 / 13$ & $<\mathrm{LOQ}$ & $<\mathrm{LOQ}$ & $<\mathrm{LOQ}$ & $<\mathrm{LOQ}$ & $<\mathrm{LOQ}$ & $<\mathrm{LOQ}$ & $<\mathrm{LOQ}$ & $<\mathrm{LOQ}$ & $<\mathrm{LOQ}$ & $<\mathrm{LOQ}$ \\
\hline $21-26 / 08 / 13$ & $<\mathrm{LOQ}$ & $<\mathrm{LOQ}$ & $<\mathrm{LOQ}$ & $<\mathrm{LOQ}$ & $<\mathrm{LOQ}$ & 0.148 & $<\mathrm{LOQ}$ & $<\mathrm{LOQ}$ & $<\mathrm{LOQ}$ & $<\mathrm{LOQ}$ \\
\hline 04-11/09/13 & $<\mathrm{LOQ}$ & $<\mathrm{LOQ}$ & $<\mathrm{LOQ}$ & $<\mathrm{LOQ}$ & $<\mathrm{LOQ}$ & $<\mathrm{LOQ}$ & $<\mathrm{LOQ}$ & $<\mathrm{LOQ}$ & $<\mathrm{LOQ}$ & $<\mathrm{LOQ}$ \\
\hline $18-25 / 09 / 13$ & $<\mathrm{LOQ}$ & $<\mathrm{LOQ}$ & $<\mathrm{LOQ}$ & $<\mathrm{LOQ}$ & $<\mathrm{LOQ}$ & 0.153 & $<\mathrm{LOQ}$ & $<\mathrm{LOQ}$ & $<\mathrm{LOQ}$ & $<\mathrm{LOQ}$ \\
\hline 02-09/10/13 & $<\mathrm{LOQ}$ & $<\mathrm{LOQ}$ & $<\mathrm{LOQ}$ & $<\mathrm{LOQ}$ & $<\mathrm{LOQ}$ & 0.547 & $<$ LOQ & $<\mathrm{LOQ}$ & $<\mathrm{LOQ}$ & $<$ LOQ \\
\hline 16-23/10/13 & $<\mathrm{LOQ}$ & $<\mathrm{LOQ}$ & $<\mathrm{LOQ}$ & $<\mathrm{LOQ}$ & $<\mathrm{LOQ}$ & 0.220 & $<\mathrm{LOQ}$ & $<\mathrm{LOQ}$ & $<\mathrm{LOQ}$ & $<\mathrm{LOQ}$ \\
\hline $30 / 10-06 / 11 / 13$ & $<\mathrm{LOQ}$ & $<\mathrm{LOQ}$ & $<\mathrm{LOQ}$ & $<\mathrm{LOQ}$ & $<\mathrm{LOQ}$ & $<\mathrm{LOQ}$ & $<\mathrm{LOQ}$ & $<\mathrm{LOQ}$ & $<\mathrm{LOQ}$ & $<\mathrm{LOQ}$ \\
\hline $13-20 / 11 / 13$ & $<\mathrm{LOQ}$ & $<\mathrm{LOQ}$ & $<\mathrm{LOQ}$ & $<\mathrm{LOQ}$ & $<\mathrm{LOQ}$ & 0.944 & $<\mathrm{LOQ}$ & $<\mathrm{LOQ}$ & $<\mathrm{LOQ}$ & $<\mathrm{LOQ}$ \\
\hline 27/11-04/12/13 & $<\mathrm{LOQ}$ & $<\mathrm{LOQ}$ & $<\mathrm{LOQ}$ & $<\mathrm{LOQ}$ & $<\mathrm{LOQ}$ & 0.551 & $<\mathrm{LOQ}$ & $<\mathrm{LOQ}$ & $<\mathrm{LOQ}$ & $<\mathrm{LOQ}$ \\
\hline $11-18 / 12 / 13$ & $<\mathrm{LOQ}$ & $<\mathrm{LOQ}$ & $<$ LOQ & $<\mathrm{LOQ}$ & $<\mathrm{LOQ}$ & 0.922 & $<\mathrm{LOQ}$ & $<\mathrm{LOQ}$ & $<\mathrm{LOQ}$ & $<\mathrm{LOQ}$ \\
\hline $5 / 12 / 13-01 / 01 / 14$ & $<\mathrm{LOQ}$ & $<\mathrm{LOQ}$ & $<\mathrm{LOQ}$ & $<\mathrm{LOQ}$ & $<\mathrm{LOQ}$ & 0.658 & $<\mathrm{LOQ}$ & $<\mathrm{LOQ}$ & $<\mathrm{LOQ}$ & $<\mathrm{LOQ}$ \\
\hline
\end{tabular}


Table S15: Concentrations of individual OCPs (in pg. $\mathrm{m}^{-3}$ ) in the gaseous phase. <LOQ indicates samples that were below the limit of quantification (in Table S7).

\begin{tabular}{|c|c|c|c|c|c|c|c|c|c|c|}
\hline Sampling time & $\alpha-\mathrm{HCI}$ & $\mathrm{HCH}$ & $-\mathrm{HCF}$ & $\mathrm{HCH}$ & $n^{\prime}-D$ & $n^{\prime}-D$ & $p^{\prime}-\mathrm{D}$ & & $n^{\prime}-\mathrm{I}$ & $n^{\prime}-\mathrm{D}$ \\
\hline $11-18 / 01 / 12$ & 1.419 & 0.191 & 1.279 & 0.133 & 0.141 & 5.282 & 0.169 & 0.250 & 0.354 & 0.265 \\
\hline $25 / 01-01 / 02 / 12$ & 2.028 & 0.190 & 1.136 & 0.095 & 0.130 & 2.812 & 0.066 & 0.141 & 0.258 & 0.281 \\
\hline $08-15 / 02 / 12$ & 1.078 & 0.150 & 0.470 & 0.098 & 0.073 & 0.612 & 0.024 & 0.118 & 0.119 & 0.130 \\
\hline $22-29 / 02 / 12$ & 2.352 & 0.206 & 3.663 & 0.167 & 0.272 & 10.366 & 0.355 & 0.548 & 0.761 & 0.537 \\
\hline $07-14 / 03 / 12$ & 1.684 & 0.198 & 2.208 & 0.108 & 0.292 & 11.552 & 0.405 & 0.665 & 0.818 & 0.578 \\
\hline $21-28 / 03 / 12$ & 1.759 & 0.189 & 3.357 & 0.123 & 0.479 & 19.750 & 0.623 & 1.081 & 1.619 & 1.399 \\
\hline 04-11/04/12 & 2.480 & 0.159 & 3.180 & 0.164 & 0.352 & 14.747 & 0.414 & 0.688 & 1.053 & 0.905 \\
\hline $18-25 / 04 / 12$ & 1.740 & 0.098 & 2.518 & 0.103 & & 12.826 & 0.361 & 0.612 & 1.065 & 936 \\
\hline $02-09 / 05 / 12$ & 1.788 & 0.256 & 7.522 & 0.220 & 0.737 & 33.746 & 1.276 & 2.565 & 3.463 & 3.307 \\
\hline $16-23 / 05 / 12$ & 3.145 & 0.255 & 5.562 & 0.100 & 348 & 12.285 & 444 & 0.708 & 1.416 & 1.180 \\
\hline $30 / 05-06 / 06 / 12$ & 1.832 & 0.182 & 7.994 & 0.169 & 0.445 & 19.230 & 0.657 & 1.070 & 2.547 & 2.307 \\
\hline $13-20 / 06 / 12$ & 2.574 & 0.343 & 9.468 & 0.260 & 0.647 & 27.765 & 0.299 & 0.442 & 4.558 & 5.556 \\
\hline 27/06-04/07/12 & 2.243 & 0.461 & 8.816 & 0.420 & 0.739 & 31.242 & 0.326 & 0.453 & 5.041 & 6.598 \\
\hline $11-18 / 07 / 12$ & 3.004 & 0.182 & 10.049 & 0.185 & 0.245 & 11.689 & 0.118 & 0.187 & 1.606 & 1.942 \\
\hline $25 / 07-01 / 08 / 12$ & 2.149 & 0.462 & 9.214 & 0.338 & 0.617 & 29.743 & 0.324 & 0.419 & 5.674 & 7.025 \\
\hline $08-1$ & 2.235 & 0.210 & 5.112 & 0.180 & & 16.873 & 169 & 0.254 & 2.381 & 2.802 \\
\hline $22-2$ & 1.886 & 0.226 & 7.798 & 0.249 & 0.453 & 25.550 & 207 & 0.313 & 3.208 & 4.299 \\
\hline $05-1$ & 1.392 & 0.243 & 5.456 & 0.179 & 0.682 & 37.298 & 343 & 0.524 & 4.757 & 5.910 \\
\hline $19-26$ & 3.331 & 0.150 & 6.585 & 0.175 & 0.667 & 40.370 & 260 & 0.339 & 4.236 & 4.771 \\
\hline $03-10$ & 4.161 & 0.194 & 8.211 & 0.179 & 0.566 & 36.428 & 243 & 0.363 & 3.429 & 4.245 \\
\hline $17-24$ & .090 & 0.190 & 7.632 & 0.263 & & 32.436 & 178 & 0.250 & 2.992 & 366 \\
\hline $31 / 11-C$ & 4.103 & 0.092 & 2.990 & 0.103 & & 15.834 & 0.095 & 154 & 1.451 & 1.621 \\
\hline $14-2$ & 9.779 & 0.322 & 8.351 & 0.214 & & 27.029 & 159 & 44 & 2.342 & 2.330 \\
\hline $28 / 11$ & 4.185 & 0.103 & 3.706 & 0.105 & & 15.392 & 107 & & 394 & .324 \\
\hline $12-$ & 3.040 & 0.069 & 2.957 & 0.086 & 210 & 5.647 & 085 & 0.142 & 0.578 & .552 \\
\hline /01/13 & 3.918 & 0.085 & 4.316 & 0.108 & 260 & 10.985 & 104 & 0.161 & 0.980 & 0.920 \\
\hline 09-16/01/13 & 3.575 & $<\mathrm{LOQ}$ & 3.177 & 0.315 & 0.168 & 5.729 & $<\mathrm{LOQ}$ & $<\mathrm{LOQ}$ & $<\mathrm{LOQ}$ & 0.329 \\
\hline $23-30$ & 2.291 & $<\mathrm{LOQ}$ & 2.031 & $<\mathrm{LOQ}$ & & 2.925 & $<\mathrm{LOQ}$ & $<\mathrm{LOQ}$ & $<\mathrm{LOQ}$ & .485 \\
\hline $06-$ & 1.767 & $<\mathrm{LOQ}$ & 1.451 & $<\mathrm{LOQ}$ & & 4.238 & $<\mathrm{LOQ}$ & $<\mathrm{LOQ}$ & $<\mathrm{LOQ}$ & 0.261 \\
\hline & 3.686 & $<\mathrm{LOQ}$ & 1.784 & $<\mathrm{LOQ}$ & & 6.506 & $<\mathrm{LOQ}$ & $<\mathrm{LOQ}$ & $<\mathrm{LOQ}$ & 0.563 \\
\hline & 4.203 & $<\mathrm{LOQ}$ & 4.277 & $<\mathrm{LOQ}$ & & 19.574 & & DQ & & .383 \\
\hline & 297 & $<\mathrm{LOQ}$ & 1.192 & $<\mathrm{LOQ}$ & & 132 & $<\mathrm{LOQ}$ & & 318 & 0.299 \\
\hline & 3.849 & $<\mathrm{LOQ}$ & 2.206 & $<\mathrm{LOQ}$ & 0.359 & 10.195 & 0.114 & $<\mathrm{LOQ}$ & 0.871 & 0.899 \\
\hline & 3.253 & 0.158 & 7.594 & 0.205 & 0.704 & 29.800 & 0.249 & $<\mathrm{LOQ}$ & 2.845 & 3.220 \\
\hline 01-08 & 3.440 & 0.218 & 6.218 & 0.165 & 0.833 & 36.576 & 0.294 & 0.473 & 3.355 & 3.484 \\
\hline & 658 & 0.126 & 5.701 & $<\mathrm{LOQ}$ & & 9.756 & .154 & & 1.681 & .857 \\
\hline 29/05-0 & 4.692 & 0.126 & 6.712 & $<\mathrm{LOQ}$ & & 18.004 & 0.204 & 0.253 & 1.959 & 1.751 \\
\hline & 1.477 & 0.264 & 4.797 & 0.267 & & & & & 3.409 & 3.777 \\
\hline $26 / 06$ & & $<\mathrm{LOQ}$ & 5.223 & & & .104 & DQ & & & 1.498 \\
\hline & & 0.336 & 6.741 & $<\mathrm{LC}$ & & 16.669 & .272 & & 3.637 & 4.033 \\
\hline & 206 & 0.592 & 9.186 & $<\mathrm{LOQ}$ & 0.950 & 38.013 & 488 & 0.441 & 6.696 & 8.037 \\
\hline & 7.074 & $<\mathrm{LOQ}$ & 21.775 & $<\mathrm{LOQ}$ & 1.420 & 71.437 & $<\mathrm{LOQ}$ & 0.892 & 9.177 & 9.985 \\
\hline & 3.556 & 0.293 & 6.445 & $<\mathrm{LOQ}$ & 0.977 & 49.387 & 0.394 & 0.322 & 5.705 & 5.813 \\
\hline 04-11/ & 4.306 & $<\mathrm{LOQ}$ & 8.588 & $<\mathrm{LOQ}$ & 0.724 & 40.787 & 0.281 & 0.253 & 4.137 & 4.279 \\
\hline $18-25 / 09 / 13$ & 3.067 & $<\mathrm{LOQ}$ & 7.702 & $<\mathrm{LOQ}$ & 0.576 & 34.714 & 0.305 & 0.270 & 3.515 & 3.912 \\
\hline $02-09 / 10 / 13$ & 3.539 & $<\mathrm{LOQ}$ & 3.285 & $<\mathrm{LOQ}$ & 0.391 & 15.309 & $<\mathrm{LOQ}$ & $<\mathrm{LOQ}$ & 1.386 & 1.435 \\
\hline & 2.951 & 0.229 & 6.366 & $<\mathrm{LOQ}$ & & 31.749 & 0.202 & & 2.962 & 2.892 \\
\hline $30 / 10$ & 3.031 & $<\mathrm{LOQ}$ & 4.464 & $<\mathrm{LOQ}$ & & 14.546 & & $<\mathrm{LOQ}$ & 1.216 & 1.014 \\
\hline & & & & & & 19.105 & $<\mathrm{LOQ}$ & & 1.543 & 1.365 \\
\hline & & & & & & & $<\mathrm{LOQ}$ & & 0.573 & 0.506 \\
\hline & 3.675 & $<\mathrm{LOQ}$ & 3.161 & $<\mathrm{LOQ}$ & 0.314 & 12.571 & $<\mathrm{LOQ}$ & $<\mathrm{LOQ}$ & 0.692 & 0.461 \\
\hline $5 / 12 / 13-01 / 01 / 14$ & 3.604 & $<\mathrm{LOQ}$ & 4.193 & $<\mathrm{LOQ}$ & 0.376 & 17.425 & $<\mathrm{LOQ}$ & $<\mathrm{LOQ}$ & 0.784 & 0.490 \\
\hline
\end{tabular}


Table S16: Results of Clausius-Clapeyron plots showing slopes (m), constants (b) and coefficient of determination $\left(\mathrm{r}^{2}\right)$ for OCPs. Compounds in bold are those where the regression parameters suggest local revolatilization as the main control on gas-phase air concentrations.

\begin{tabular}{c|ccccc} 
& $\mathrm{N}$ & $\mathrm{m}$ & $\mathrm{b}$ & $\mathrm{r}^{2}$ & $\mathrm{p}$ \\
\hline$\alpha-\mathrm{HCH}$ & 52 & -141.6 & 24.00 & $<0.01$ & $<0.819$ \\
$\boldsymbol{\beta}$-HCH & $\mathbf{3 6}$ & $\mathbf{- 3 1 4 8}$ & $\mathbf{1 6 . 0 3}$ & $\mathbf{0 . 4 0}$ & $<\mathbf{0 . 0 1}$ \\
$\boldsymbol{\gamma}$-HCH & $\mathbf{5 2}$ & $\mathbf{- 6 0 9 7}$ & $\mathbf{- 2 . 3 7 3}$ & $\mathbf{0 . 7 3}$ & $<\mathbf{0 . 0 1}$ \\
$\boldsymbol{\delta}$-HCH & $\mathbf{3 0}$ & $\mathbf{- 2 7 9 2}$ & $\mathbf{- 1 7 . 4 2}$ & $\mathbf{0 . 4 3}$ & $<\mathbf{0 . 0 1}$ \\
$\boldsymbol{o}, \boldsymbol{p}^{\prime}$-DDE & $\mathbf{5 0}$ & $\mathbf{- 4 7 9 7}$ & $\mathbf{- 9 . 4 8 4}$ & $\mathbf{0 . 6 2}$ & $<\mathbf{0 . 0 1}$ \\
$\boldsymbol{p}, \boldsymbol{p}^{\prime}$-DDE & $\mathbf{5 2}$ & $\mathbf{- 7 2 2 5}$ & $\mathbf{2 . 8 2 3}$ & $\mathbf{0 . 6 8}$ & $<\mathbf{0 . 0 1}$ \\
$\boldsymbol{o}, \boldsymbol{p}^{\prime}$-DDD & $\mathbf{3 8}$ & $\mathbf{- 5 0 6 0}$ & $\mathbf{- 9 . 2 1 7}$ & $\mathbf{0 . 4 4}$ & $<\mathbf{0 . 0 1}$ \\
$\boldsymbol{p}, \boldsymbol{p}^{\prime}$-DDD & $\mathbf{3 9}$ & $\mathbf{- 3 3 5 3}$ & $\mathbf{- 0 . 2 3 4 6}$ & $\mathbf{0 . 2 3}$ & $<\mathbf{0 . 0 1}$ \\
$\boldsymbol{o}, \boldsymbol{p}^{\prime}$-DDT & $\mathbf{4 8}$ & $\mathbf{- 8 7 2 9}$ & $\mathbf{5 . 7 7 4}$ & $\mathbf{0 . 8 1}$ & $<\mathbf{0 . 0 1}$ \\
$\boldsymbol{p}, \boldsymbol{p}^{\prime}$-DDT & $\mathbf{5 2}$ & $\mathbf{- 9 8 0 2}$ & $\mathbf{9 . 5 7 0}$ & $\mathbf{0 . 8 4}$ & $<\mathbf{0 . 0 1}$ \\
\hline
\end{tabular}

Table S17: Results of Clausius-Clapeyron plots showing slopes (m), constants (b) and coefficient of determination $\left(\mathrm{r}^{2}\right)$ for CUPs. Compounds in bold are those where regression parameters suggest local revolatilization as the main control on gas-phase air concentrations.

\begin{tabular}{c|ccccc} 
& $\mathrm{N}$ & $\mathrm{m}$ & $\mathrm{b}$ & $\mathrm{r}^{2}$ & $\mathrm{p}$ \\
\hline Metazachlor & 31 & -6855 & 1.381 & 0.06 & $<0.182$ \\
Isoproturon & 25 & -5313 & -7.936 & 0.09 & $<0.154$ \\
Chlorpyrifos & 44 & -4922 & -6.857 & 0.06 & $<0.109$ \\
Terbuthylazine & $\mathbf{3 4}$ & $\mathbf{- 2 0 6 4 7}$ & $\mathbf{4 7 . 2 6}$ & $\mathbf{0 . 4 8}$ & $<\mathbf{0 . 0 1}$ \\
S-Metolachlor & $\mathbf{3 4}$ & $\mathbf{- 1 5 7 9 1}$ & $\mathbf{3 0 . 4 4}$ & $\mathbf{0 . 2 2}$ & $<\mathbf{0 . 0 1}$ \\
Acetochlor & 23 & 4408 & -39.56 & 0.01 & $<0.632$ \\
Dimethachlor & 18 & -15721 & 30.19 & 0.19 & $<0.075$ \\
\hline
\end{tabular}


Table S18: Octanol-air partitioning coefficients, $\mathrm{K}_{\mathrm{OA}}$, of OCPs and CUPs at $25^{\circ} \mathrm{C}, \mathrm{T}_{\min }\left(-10.3^{\circ} \mathrm{C}\right.$ for OCPs and $-13.3^{\circ} \mathrm{C}$ for CUPs $)$ and $\mathrm{T}_{\max }\left(22.7^{\circ} \mathrm{C}\right.$ for OCPs and $22.8^{\circ} \mathrm{C}$ for CUPs). Extrapolation: see text.

\begin{tabular}{|c|c|c|c|c|c|}
\hline \multirow{2}{*}{ 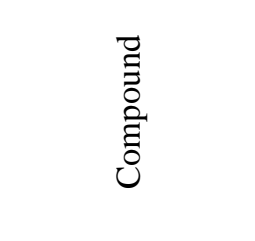 } & \multirow{2}{*}{ 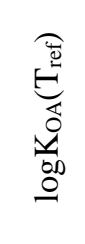 } & \multirow{2}{*}{ 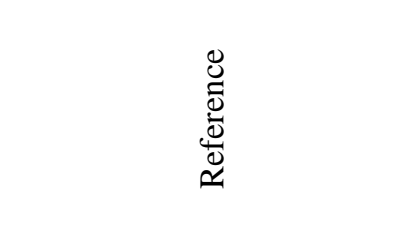 } & \multicolumn{3}{|c|}{ Temperature-corrected $\log \mathrm{K}_{\mathrm{OA}}(\mathrm{T})$} \\
\hline & & & 舄 & 总 & 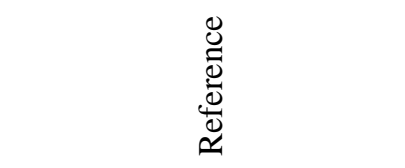 \\
\hline Acetochlor & 9.07 & (Coscollà et al., 2013b) & 11.2 & 9.16 & Extrapolated \\
\hline Alachlor & 9.98 & (Coscollà et al., 2013b) & 12.3 & 10.1 & Extrapolated \\
\hline Atrazine & 9.62 & (Götz et al., 2007) & 11.9 & 9.72 & Extrapolated \\
\hline Azinphos Methyl & 8.76 & (Coscollà et al., 2013a) & 10.8 & 8.85 & Extrapolated \\
\hline Carbendazim & 10.6 & (Coscollà et al., 2013a) & 13.1 & 10.7 & Extrapolated \\
\hline Chlorotoluron & 10.6 & (Götz et al., 2007) & 13.1 & 10.7 & Extrapolated \\
\hline Chlorpyrifos & 8.41 & (Odabasi and Cetin, 2012b) & 10.3 & 8.50 & (Odabasi and Cetin, 2012b) \\
\hline Diazinon & 9.14 & (Coscollà et al., 2013b) & 11.3 & 9.24 & Extrapolated \\
\hline Dimethachlor & 9.34 & (U.S. EPA, 2012) & 11.5 & 9.44 & Extrapolated \\
\hline Dimethoate & 9.15 & (Coscollà et al., 2013a) & 11.3 & 9.25 & Extrapolated \\
\hline Disulfoton & 8.07 & (U.S. EPA, 2012) & 10.0 & 8.16 & Extrapolated \\
\hline Diuron & 10.4 & (Coscollà et al., 2013a) & 12.8 & 10.5 & Extrapolated \\
\hline Fenitrothion & 7.72 & (U.S. EPA, 2012) & 9.58 & 7.80 & Extrapolated \\
\hline Fenpropimorph & 8.93 & (Coscollà et al., 2013b) & 11.0 & 9.02 & Extrapolated \\
\hline Fonofos & 7.48 & (U.S. EPA, 2012) & 9.30 & 7.56 & Extrapolated \\
\hline Isoproturon & 11.2 & (Götz et al., 2007) & 13.8 & 11.3 & Extrapolated \\
\hline Malathion & 9.06 & (Coscollà et al., 2013b) & 11.2 & 9.15 & Extrapolated \\
\hline Metamitron & 11.2 & (U.S. EPA, 2012) & 13.8 & 11.4 & Extrapolated \\
\hline Metazachlor & 9.76 & (Coscollà et al., 2013b) & 12.0 & 9.86 & Extrapolated \\
\hline Metribuzin & 10.0 & (U.S. EPA, 2012) & 12.3 & 10.1 & Extrapolated \\
\hline Prochloraz & 13.6 & (U.S. EPA, 2012) & 16.6 & 13.7 & Extrapolated \\
\hline Pyrazon & 9.01 & (U.S. EPA, 2012) & 11.1 & 9.10 & Extrapolated \\
\hline Simazine & 9.59 & (Götz et al., 2007) & 11.8 & 9.69 & Extrapolated \\
\hline S-Metolachlor & 9.33 & (U.S. EPA, 2012) & 11.5 & 9.43 & Extrapolated \\
\hline Temephos & 13.1 & (U.S. EPA, 2012) & 16.0 & 13.2 & Extrapolated \\
\hline Terbufos & 7.49 & (U.S. EPA, 2012) & 9.30 & 7.57 & Extrapolated \\
\hline Terbuthylazine & 9.03 & (Coscollà et al., 2013a) & 11.2 & 9.12 & Extrapolated \\
\hline$\alpha-\mathrm{HCH}$ & 7.61 & (Shoeib and Harner, 2002b) & 9.06 & 7.69 & (Shoeib and Harner, 2002b) \\
\hline$\beta-\mathrm{HCH}$ & 8.88 & (Shoeib and Harner, 2002b) & 11.1 & 9.00 & (Shoeib and Harner, 2002b) \\
\hline$\gamma-\mathrm{HCH}$ & 7.85 & (Shoeib and Harner, 2002b) & 9.38 & 7.93 & (Shoeib and Harner, 2002b) \\
\hline$\delta-\mathrm{HCH}$ & 8.84 & (Shoeib and Harner, 2002b) & 11.0 & 8.97 & (Shoeib and Harner, 2002b) \\
\hline$o, p^{\prime}-\mathrm{DDE}$ & 9.26 & (Zhang et al., 2009) & 10.9 & 9.12 & (Zhang et al., 2009) \\
\hline$p, p^{\prime}-\mathrm{DDE}$ & 9.68 & (Shoeib and Harner, 2002b) & 12.0 & 9.80 & (Shoeib and Harner, 2002b) \\
\hline$o, p^{\prime}-\mathrm{DDD}$ & 9.57 & (Zhang et al., 2009) & 11.3 & 9.42 & (Zhang et al., 2009) \\
\hline$p, p^{\prime}-\mathrm{DDD}$ & 10.1 & (Shoeib and Harner, 2002b) & 12.0 & 10.2 & (Shoeib and Harner, 2002b) \\
\hline$o, p^{\prime}-\mathrm{DDT}$ & 9.45 & (Shoeib and Harner, 2002b) & 11.5 & 9.57 & (Shoeib and Harner, 2002b) \\
\hline$p, p^{\prime}-\mathrm{DDT}$ & 9.82 & (Shoeib and Harner, 2002b) & 11.9 & 9.93 & (Shoeib and Harner, 2002b) \\
\hline
\end{tabular}


Table S19: Particulate concentrations of individual OCPs (in pg. $\mathrm{m}^{-3}$ ) according to particle size (in $\mu \mathrm{m}$ ) at the rural and the urban sites. N/D indicates that a compound was not detected. Numbers in bolds represent the particle size fraction with the highest concentration.

\begin{tabular}{|c|c|c|c|c|c|c|c|c|c|c|c|c|c|}
\hline & & $\begin{array}{l}\text { Size fraction } \\
(\mu \mathrm{m})\end{array}$ & $\alpha-\mathrm{HCH}$ & $\beta-\mathrm{HCH}$ & $\gamma-\mathrm{HCH}$ & $\delta-\mathrm{HCH}$ & $\begin{array}{l}o, p^{\prime}- \\
\text { DDE }\end{array}$ & $\begin{array}{l}p, p^{\prime}- \\
\text { DDE }\end{array}$ & $\begin{array}{l}o, p^{\prime}- \\
\text { DDD }\end{array}$ & $\begin{array}{l}p, p^{\prime}- \\
\text { DDD }\end{array}$ & $\begin{array}{l}o, p^{\prime}- \\
\text { DDT }\end{array}$ & $\begin{array}{l}p, p^{\prime}- \\
\text { DDT }\end{array}$ & $\Sigma O C P s$ \\
\hline \multirow{24}{*}{ 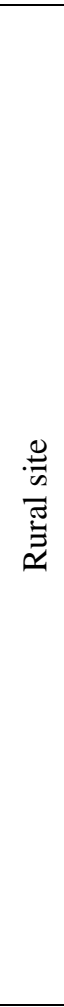 } & \multirow{6}{*}{ 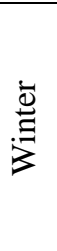 } & $7.2-10$ & 0.01 & 0.05 & 0.17 & 0.04 & 0.05 & 0.67 & 0.03 & 0.19 & 0.31 & 1.47 & 2.99 \\
\hline & & $3.0-7.2$ & 0.16 & 0.02 & 0.33 & 0.08 & 0.05 & 0.91 & 0.06 & 0.37 & 0.25 & 1.38 & 3.61 \\
\hline & & $1.5-3.0$ & 0.18 & 0.13 & 0.33 & 0.04 & 0.08 & 1.01 & 0.06 & 0.43 & 0.24 & 1.51 & 4.01 \\
\hline & & $0.95-1.5$ & 0.09 & 0.09 & 0.23 & 0.10 & 0.17 & 2.71 & 0.27 & 1.73 & 0.48 & 3.29 & 9.16 \\
\hline & & $0.49-0.95$ & N/D & 0.06 & 0.17 & 0.07 & 0.24 & 4.00 & 0.32 & 2.24 & 0.55 & 4.04 & 11.69 \\
\hline & & $<0.49$ & 0.44 & 0.18 & 0.56 & 0.36 & 0.18 & 3.67 & 0.27 & 2.04 & 1.02 & 9.88 & 18.6 \\
\hline & \multirow{6}{*}{ 号 } & $7.2-10$ & 0.22 & 0.04 & 0.49 & 0.24 & 0.05 & 1.25 & 0.04 & 0.11 & 0.20 & 0.54 & 3.18 \\
\hline & & $3.0-7.2$ & 0.11 & N/D & 0.37 & 0.16 & 0.02 & 0.92 & 0.02 & 0.12 & 0.14 & 0.48 & 2.34 \\
\hline & & $1.5-3.0$ & 0.19 & N/D & 0.48 & 0.19 & 0.03 & 1.17 & 0.03 & 0.15 & 0.16 & 0.69 & 3.09 \\
\hline & & $0.95-1.5$ & 0.33 & 0.02 & 0.68 & 0.24 & 0.04 & 1.65 & 0.09 & 0.25 & 0.30 & 1.71 & 5.31 \\
\hline & & $0.49-0.95$ & 0.05 & 0.06 & 0.26 & 0.03 & 0.05 & 2.43 & 0.08 & 0.46 & 0.41 & 2.59 & 6.42 \\
\hline & & $<0.49$ & 0.04 & 1.27 & 0.32 & 0.17 & 0.06 & 1.94 & 0.16 & 0.23 & 0.69 & 4.69 & 9.57 \\
\hline & \multirow{6}{*}{$\begin{array}{l}\bar{\Xi} \\
\stackrel{\Xi}{\Xi} \\
\vdots \\
\bar{\Xi}\end{array}$} & $7.2-10$ & 0.05 & N/D & 0.25 & N/D & 0.03 & 1.17 & 0.13 & 0.42 & 0.06 & N/D & 2.11 \\
\hline & & $3.0-7.2$ & 0.07 & N/D & 0.26 & N/D & 0.03 & 1.01 & 0.13 & 0.50 & 0.06 & 0.09 & 2.15 \\
\hline & & $1.5-3.0$ & 0.26 & 0.01 & 0.09 & N/D & 0.02 & 0.73 & 0.08 & 0.37 & N/D & N/D & 1.56 \\
\hline & & $0.95-1.5$ & 0.10 & N/D & 0.15 & 0.05 & 0.01 & 0.65 & 0.08 & 0.35 & N/D & 0.02 & 1.41 \\
\hline & & $0.49-0.95$ & 0.22 & 0.14 & 0.22 & 0.10 & 0.02 & 1.11 & 0.13 & 0.49 & 0.04 & 0.23 & 2.7 \\
\hline & & $<0.49$ & 0.09 & 0.40 & 0.17 & 0.06 & 0.07 & 2.26 & 0.15 & 0.77 & 0.15 & 0.35 & 4.47 \\
\hline & \multirow{6}{*}{ 害 } & $7.2-10$ & 0.01 & 0.03 & 0.04 & 0.00 & 0.01 & 0.65 & 0.07 & 0.26 & 0.00 & N/D & 1.07 \\
\hline & & $3.0-7.2$ & 0.01 & N/D & 0.03 & N/D & 0.03 & 1.57 & 0.13 & 0.70 & 0.01 & N/D & 2.48 \\
\hline & & $1.5-3.0$ & N/D & 0.20 & 0.06 & 0.04 & 0.04 & 2.18 & 0.18 & 1.03 & N/D & 0.00 & 3.73 \\
\hline & & $0.95-1.5$ & 0.13 & 0.16 & 0.15 & 0.11 & 0.10 & 5.31 & 0.41 & 2.63 & 0.07 & 0.18 & 9.25 \\
\hline & & $0.49-0.95$ & 0.02 & 0.12 & 0.06 & 0.04 & 0.13 & 8.17 & 0.60 & 4.21 & 0.18 & 0.52 & 14.05 \\
\hline & & $<0.49$ & 0.05 & 0.09 & 0.03 & N/D & 0.10 & 6.11 & 0.47 & 3.53 & 0.35 & 1.39 & 12.12 \\
\hline \multirow{24}{*}{ 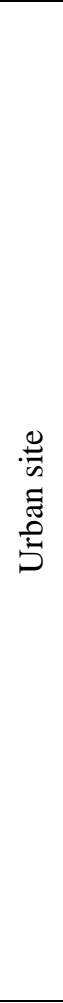 } & \multirow{6}{*}{ 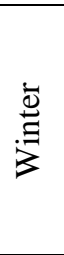 } & $7.2-10$ & N/D & 0.89 & 0.11 & N/D & 0.07 & 0.64 & 0.13 & 0.40 & 0.03 & N/D & 2.27 \\
\hline & & $3.0-7.2$ & N/D & 0.53 & 0.07 & N/D & 0.08 & 0.75 & 0.15 & 0.39 & 0.05 & 0.08 & 2.10 \\
\hline & & $1.5-3.0$ & N/D & 0.24 & 0.12 & N/D & 0.12 & 1.12 & 0.19 & 0.58 & 0.06 & 0.11 & 2.54 \\
\hline & & $0.95-1.5$ & N/D & 0.20 & 0.11 & 0.02 & 0.16 & 1.75 & 0.29 & 1.02 & 0.13 & 0.13 & 3.81 \\
\hline & & $0.49-0.95$ & 0.01 & 0.12 & 0.12 & N/D & 0.19 & 1.92 & 0.35 & 1.37 & 0.08 & 0.15 & 4.31 \\
\hline & & $<0.49$ & 0.22 & 0.17 & 0.33 & 0.18 & 0.19 & 2.29 & 0.49 & 1.85 & 0.41 & 0.40 & 6.53 \\
\hline & \multirow{6}{*}{ 誓 } & $7.2-10$ & 0.02 & 0.56 & 0.09 & 0.03 & 0.05 & 0.68 & 0.16 & 0.58 & 0.06 & 0.06 & 2.29 \\
\hline & & $3.0-7.2$ & 0.10 & 0.75 & 0.15 & 0.06 & 0.07 & 0.93 & 0.20 & 0.71 & 0.06 & 0.07 & 3.10 \\
\hline & & $1.5-3.0$ & N/D & N/D & 0.03 & N/D & 0.04 & 0.68 & 0.16 & 0.56 & 0.04 & 0.03 & 1.54 \\
\hline & & $0.95-1.5$ & N/D & 0.08 & 0.03 & N/D & 0.05 & 0.77 & 0.18 & 0.65 & 0.05 & 0.06 & 1.87 \\
\hline & & $0.49-0.95$ & 0.01 & N/D & 0.08 & N/D & 0.08 & 1.32 & 0.31 & 1.20 & 0.09 & 0.14 & 3.23 \\
\hline & & $<0.49$ & 0.07 & 0.52 & 0.32 & N/D & 0.13 & 2.05 & 0.46 & 1.89 & 0.25 & 0.46 & 6.15 \\
\hline & \multirow{6}{*}{$\begin{array}{l}\dot{\Phi} \\
\Xi \\
\Xi \\
\tilde{\Xi}\end{array}$} & $7.2-10$ & N/D & 0.48 & 0.04 & $\mathrm{~N} / \mathrm{D}$ & 0.05 & 0.68 & 0.17 & 0.57 & 0.07 & 0.06 & 2.12 \\
\hline & & $3.0-7.2$ & 0.05 & 0.04 & 0.08 & N/D & 0.07 & 1.11 & 0.27 & 1.07 & 0.13 & 0.21 & 3.03 \\
\hline & & $1.5-3.0$ & 0.14 & 0.16 & 0.11 & N/D & 0.05 & 0.69 & 0.18 & 0.66 & 0.12 & 0.25 & 2.36 \\
\hline & & $0.95-1.5$ & 0.11 & 0.07 & 0.09 & N/D & 0.06 & 0.80 & 0.22 & 0.80 & 0.09 & 0.12 & 2.36 \\
\hline & & $0.49-0.95$ & 0.23 & 0.98 & 0.26 & 0.14 & 0.07 & 0.94 & 0.24 & 0.99 & 0.13 & 0.25 & 4.23 \\
\hline & & $<0.49$ & 0.19 & 0.41 & 0.38 & 0.23 & 0.14 & 1.95 & 0.43 & 1.56 & 0.30 & 0.39 & 5.98 \\
\hline & \multirow{6}{*}{ 志 } & $7.2-10$ & 0.02 & 0.49 & 0.17 & 0.04 & 0.10 & 2.07 & 0.28 & 1.26 & 0.17 & 0.33 & 4.93 \\
\hline & & $3.0-7.2$ & N/D & 0.47 & 0.07 & N/D & 0.12 & 2.08 & 0.32 & 1.15 & 0.19 & 0.24 & 4.64 \\
\hline & & $1.5-3.0$ & N/D & 0.35 & 0.07 & N/D & 0.10 & 1.81 & 0.33 & 1.24 & 0.12 & 0.28 & 4.30 \\
\hline & & $0.95-1.5$ & N/D & 0.02 & 0.03 & N/D & 0.15 & 2.77 & 0.45 & 2.16 & 0.20 & 0.50 & 6.28 \\
\hline & & $0.49-0.95$ & N/D & 0.03 & 0.07 & N/D & 0.22 & 4.06 & 0.68 & 3.49 & 0.46 & 1.14 & 10.15 \\
\hline & & $<0.49$ & N/D & 0.02 & 0.18 & 0.06 & 0.22 & 4.77 & 0.92 & 3.92 & 0.54 & 1.27 & 11.9 \\
\hline
\end{tabular}


Table S20: Particulate concentrations of individual CUPs (in pg. $\mathrm{m}^{-3}$ ) according to particle size (in $\left.\mu \mathrm{m}\right)$ at the rural and urban sites. Disulfoton was not included in this dataset. N/D indicates compounds that were not detected, <LOD indicates compounds that were below the limit of detection and <LOQ indicates samples that were below the limit of quantification. LODs and LOQs are found in Table S7. Numbers in bolds represent the particle size fraction with the highest concentration.

\begin{tabular}{|c|c|c|c|c|c|c|c|c|c|c|c|c|c|c|}
\hline & & 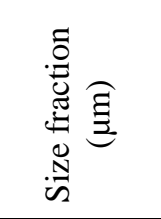 & $\begin{array}{l}\frac{\tilde{0}}{\overline{0}} \\
\frac{0}{0} \\
\frac{0}{0} \\
0\end{array}$ & $\begin{array}{l}\frac{\grave{0}}{\bar{J}} \\
\frac{\tilde{J}}{4} \\
\frac{\pi}{4}\end{array}$ & 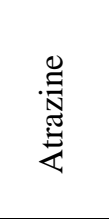 & 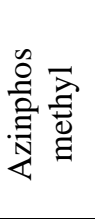 & 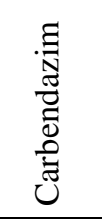 & 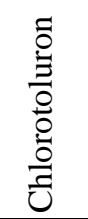 & 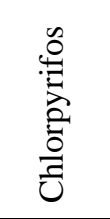 & 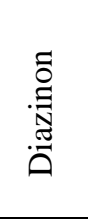 & 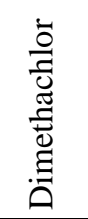 & 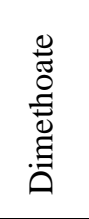 & 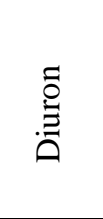 & 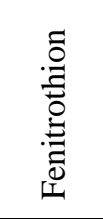 \\
\hline \multirow{24}{*}{ 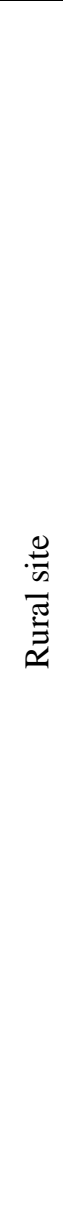 } & \multirow{6}{*}{$\stackrel{\vec{\omega}}{\stackrel{\vec{D}}{3}}$} & $7.2-10$ & 0.05 & $<\mathrm{LOD}$ & 0.21 & N/D & N/D & N/D & $<\mathrm{LOQ}$ & 0.94 & N/D & N/D & $<\mathrm{LOQ}$ & $<\mathrm{LOD}$ \\
\hline & & $3.0-7.2$ & 0.07 & 0.16 & 0.31 & N/D & N/D & N/D & $<\mathrm{LOQ}$ & 0.76 & N/D & N/D & 0.06 & $<$ LOD \\
\hline & & $1.5-3.0$ & 0.06 & 0.31 & 0.24 & N/D & N/D & N/D & N/D & 0.27 & N/D & N/D & $<\mathrm{LOQ}$ & $<$ LOD \\
\hline & & $0.95-1.5$ & $<\mathrm{LOQ}$ & $<\mathrm{LOD}$ & 0.96 & N/D & N/D & N/D & $<\mathrm{LOQ}$ & 0.32 & N/D & N/D & 0.21 & $<$ LOD \\
\hline & & $0.49-0.95$ & 0.09 & $<\mathrm{LOQ}$ & 0.56 & N/D & N/D & N/D & $\mathrm{N} / \mathrm{D}$ & N/D & N/D & N/D & 0.29 & $<\mathrm{LOD}$ \\
\hline & & $<0.49$ & 0.26 & $<\mathrm{LOD}$ & N/D & N/D & N/D & N/D & 0.15 & N/D & N/D & N/D & 0.96 & $<\mathrm{LOD}$ \\
\hline & \multirow{6}{*}{$\begin{array}{l}\text { 足 } \\
\text { 足 }\end{array}$} & $7.2-10$ & 20.91 & $<\mathrm{LOQ}$ & N/D & 0.05 & 0.44 & 0.71 & 0.27 & 1.20 & N/D & N/D & 0.11 & $<$ LOD \\
\hline & & $3.0-7.2$ & 32.96 & $<\mathrm{LOQ}$ & 0.04 & 0.06 & 1.38 & 1.63 & 0.15 & 1.87 & N/D & N/D & 0.24 & $<$ LOD \\
\hline & & $1.5-3.0$ & 31.67 & 0.12 & 0.04 & 0.06 & 0.59 & 0.83 & 0.28 & 0.71 & N/D & 0.01 & 0.37 & $<$ LOD \\
\hline & & $0.95-1.5$ & 17.81 & $<\mathrm{LOD}$ & N/D & N/D & 0.19 & N/D & 0.20 & 0.30 & N/D & 0.09 & 0.34 & $<$ LOD \\
\hline & & $0.49-0.95$ & 26.33 & 0.48 & 0.04 & N/D & N/D & N/D & 0.24 & 0.25 & N/D & 0.26 & 0.52 & $<\mathrm{LOD}$ \\
\hline & & $<0.49$ & 144.51 & $<\mathrm{LOD}$ & 0.34 & N/D & $<\mathrm{LOQ}$ & N/D & 0.67 & 0.95 & N/D & 0.53 & 2.29 & $<\mathrm{LOD}$ \\
\hline & \multirow{6}{*}{$\begin{array}{l}\overrightarrow{\mathrm{D}} \\
\text { 音 } \\
\text { 员 }\end{array}$} & $7.2-10$ & 1.99 & 0.23 & 0.05 & N/D & 0.82 & N/D & $<\mathrm{LOQ}$ & 1.57 & N/D & N/D & 1.05 & $<$ LOD \\
\hline & & $3.0-7.2$ & 2.91 & 0.12 & 0.13 & 0.07 & 2.04 & N/D & $<\mathrm{LOQ}$ & 0.80 & N/D & N/D & 1.73 & $<$ LOD \\
\hline & & $1.5-3.0$ & 4.26 & 0.18 & 0.10 & N/D & 0.87 & N/D & $<\mathrm{LOQ}$ & 1.31 & N/D & N/D & 2.40 & $<$ LOD \\
\hline & & $0.95-1.5$ & 3.84 & 0.27 & 0.03 & N/D & 0.40 & N/D & $<\mathrm{LOQ}$ & 1.75 & N/D & N/D & 2.53 & $<$ LOD \\
\hline & & $0.49-0.95$ & 1.26 & 0.30 & N/D & N/D & 0.40 & N/D & $<\mathrm{LOQ}$ & 0.88 & N/D & N/D & 3.40 & $<$ LOD \\
\hline & & $<0.49$ & 1.10 & 0.79 & N/D & N/D & 0.63 & N/D & 0.11 & 0.29 & N/D & N/D & 16.72 & $<$ LOD \\
\hline & \multirow{6}{*}{$\begin{array}{l}\text { 吾 } \\
\text { 宼 }\end{array}$} & $7.2-10$ & 0.40 & N/D & $<\mathrm{LOQ}$ & N/D & 0.12 & N/D & $<\mathrm{LOQ}$ & 0.69 & N/D & N/D & 0.16 & $\mathrm{~N} / \mathrm{D}$ \\
\hline & & $3.0-7.2$ & 0.39 & N/D & $<\mathrm{LOD}$ & N/D & 0.01 & N/D & $\mathrm{N} / \mathrm{D}$ & 0.51 & N/D & N/D & 0.25 & N/D \\
\hline & & $1.5-3.0$ & 0.57 & $<\mathrm{LOD}$ & $\mathrm{N} / \mathrm{D}$ & 0.06 & 1.48 & 0.33 & $<\mathrm{LOQ}$ & 0.95 & 0.11 & N/D & 0.36 & N/D \\
\hline & & $0.95-1.5$ & 0.26 & N/D & N/D & N/D & N/D & N/D & $<\mathrm{LOQ}$ & 1.16 & N/D & N/D & 0.76 & $<$ LOD \\
\hline & & $0.49-0.95$ & 0.21 & 1.47 & N/D & N/D & N/D & N/D & $<\mathrm{LOQ}$ & N/D & N/D & N/D & 1.41 & N/D \\
\hline & & $<0.49$ & 0.64 & N/D & N/D & N/D & 0.25 & N/D & $<\mathrm{LOQ}$ & 3.29 & 0.43 & N/D & 5.11 & N/D \\
\hline
\end{tabular}




\begin{tabular}{|c|c|c|c|c|c|c|c|c|c|c|c|c|c|c|}
\hline \multirow{24}{*}{ 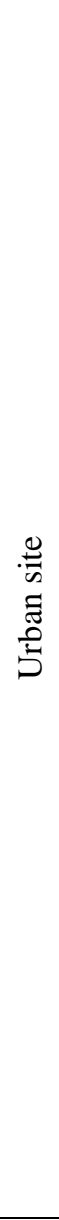 } & \multirow{6}{*}{$\stackrel{\stackrel{ \pm}{\Xi}}{i}$} & $7.2-10$ & 0.03 & 0.50 & 0.25 & 0.05 & N/D & N/D & N/D & 0.90 & N/D & 0.02 & N/D & N/D \\
\hline & & $3.0-7.2$ & 0.04 & 0.21 & 0.06 & N/D & N/D & N/D & N/D & 0.07 & N/D & N/D & 0.03 & N/D \\
\hline & & $1.5-3.0$ & 0.06 & N/D & 0.40 & N/D & N/D & N/D & N/D & N/D & N/D & N/D & 0.07 & N/D \\
\hline & & $0.95-1.5$ & 0.08 & N/D & 0.27 & N/D & N/D & N/D & N/D & 0.23 & N/D & N/D & $<\mathrm{LOQ}$ & N/D \\
\hline & & $0.49-0.95$ & 0.21 & N/D & 0.56 & N/D & N/D & N/D & $<\mathrm{LOQ}$ & N/D & N/D & N/D & 0.06 & 6.22 \\
\hline & & $<0.49$ & 0.39 & 10.47 & 1.03 & N/D & N/D & N/D & 0.66 & N/D & N/D & N/D & 0.20 & N/D \\
\hline & \multirow{6}{*}{ 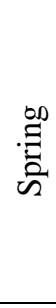 } & $7.2-10$ & 2.17 & 0.17 & 0.35 & N/D & 0.71 & 0.63 & $<\mathrm{LOQ}$ & 0.61 & N/D & N/D & 0.09 & N/D \\
\hline & & $3.0-7.2$ & 1.59 & 0.25 & 0.04 & N/D & 1.05 & 1.06 & N/D & 0.05 & N/D & N/D & 0.14 & N/D \\
\hline & & $1.5-3.0$ & 3.67 & 0.21 & 0.08 & N/D & 0.62 & 0.08 & 0.11 & 1.18 & N/D & N/D & 0.13 & N/D \\
\hline & & $0.95-1.5$ & 2.61 & N/D & N/D & N/D & N/D & N/D & 0.31 & 0.44 & N/D & N/D & 0.10 & N/D \\
\hline & & $0.49-0.95$ & 1.14 & N/D & 0.06 & N/D & N/D & N/D & 0.17 & N/D & N/D & N/D & 0.16 & N/D \\
\hline & & $<0.49$ & 6.95 & N/D & 0.36 & N/D & 0.15 & N/D & 0.30 & N/D & N/D & N/D & 1.39 & N/D \\
\hline & \multirow{6}{*}{ 㐫 } & $7.2-10$ & 0.61 & N/D & N/D & N/D & 1.21 & N/D & $<\mathrm{LOQ}$ & 1.40 & 0.35 & N/D & 0.06 & N/D \\
\hline & & $3.0-7.2$ & 0.44 & $<\mathrm{LOQ}$ & $<\mathrm{LOQ}$ & N/D & 0.98 & N/D & 0.11 & 0.96 & 0.51 & N/D & 0.17 & N/D \\
\hline & & $1.5-3.0$ & 1.76 & N/D & $<\mathrm{LOD}$ & N/D & 0.70 & N/D & 0.12 & 1.18 & 0.40 & N/D & 1.16 & N/D \\
\hline & & $0.95-1.5$ & 0.62 & N/D & 0.02 & N/D & 0.20 & $\mathrm{~N} / \mathrm{D}$ & 0.06 & 0.55 & N/D & N/D & 0.21 & N/D \\
\hline & & $0.49-0.95$ & 0.68 & N/D & N/D & N/D & 1.66 & N/D & 1.06 & 0.82 & 0.29 & N/D & 0.67 & N/D \\
\hline & & $<0.49$ & 3.53 & 0.61 & 0.14 & N/D & N/D & N/D & 0.31 & 2.14 & 7.32 & N/D & 3.51 & N/D \\
\hline & \multirow{6}{*}{ 声 } & $7.2-10$ & 0.34 & 0.25 & N/D & N/D & 0.06 & N/D & $<$ LOQ & 1.01 & N/D & $\mathrm{N} / \mathrm{D}$ & 0.05 & N/D \\
\hline & & $3.0-7.2$ & 0.47 & 0.37 & 0.11 & N/D & 0.36 & 0.19 & N/D & N/D & $<\mathrm{LOQ}$ & N/D & 0.15 & N/D \\
\hline & & $1.5-3.0$ & 0.36 & N/D & N/D & N/D & 0.21 & N/D & N/D & 1.47 & 0.10 & N/D & N/D & N/D \\
\hline & & $0.95-1.5$ & 0.24 & 0.53 & N/D & N/D & N/D & N/D & N/D & 1.35 & $<\mathrm{LOD}$ & N/D & $<\mathrm{LOQ}$ & $<\mathrm{LOD}$ \\
\hline & & $0.49-0.95$ & 0.09 & 0.88 & 0.10 & N/D & 0.01 & N/D & N/D & N/D & N/D & N/D & 0.26 & N/D \\
\hline & & $<0.49$ & 0.22 & 0.61 & 0.11 & N/D & N/D & N/D & $<\mathrm{LOQ}$ & 0.79 & 0.09 & N/D & 0.29 & N/D \\
\hline
\end{tabular}


Table S20 (continued): Particulate concentrations of individual CUPs (in pg. $\mathrm{m}^{-3}$ ) according to particle size (in $\mu \mathrm{m}$ ) at the rural and urban sites.

\begin{tabular}{|c|c|c|c|c|c|c|c|c|c|c|c|c|c|c|c|c|c|}
\hline & & 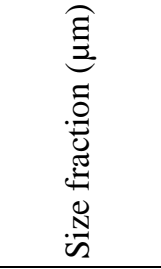 & 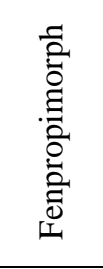 & $\begin{array}{l}0 \\
0 \\
0 \\
0 \\
0\end{array}$ & 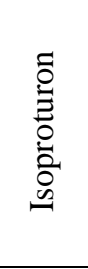 & 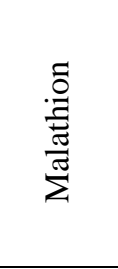 & 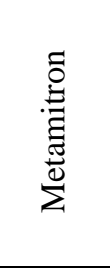 & 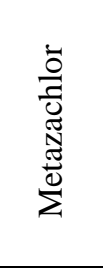 & 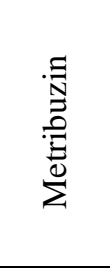 & 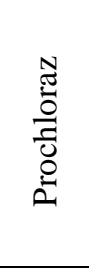 & 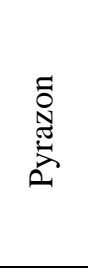 & 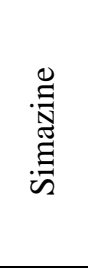 & 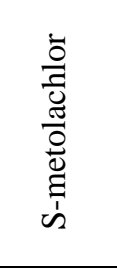 & 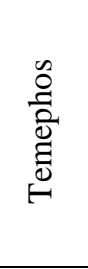 & $\begin{array}{l}\stackrel{0}{0} \\
\stackrel{0}{0} \\
\stackrel{0}{0} \\
\qquad\end{array}$ & 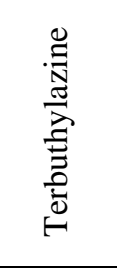 & $\overbrace{\omega}^{\infty}$ \\
\hline \multirow{24}{*}{ 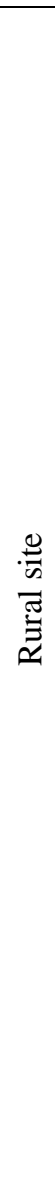 } & \multirow{6}{*}{ 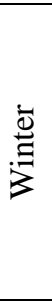 } & $7.2-10$ & 0.03 & 0.04 & 0.03 & $<\mathrm{LOQ}$ & $<\mathrm{LOD}$ & N/D & $<\mathrm{LOD}$ & N/D & N/D & 0.19 & N/D & 0.03 & N/D & N/D & 1.52 \\
\hline & & $3.0-7.2$ & 0.03 & 0.05 & 0.11 & 0.08 & $<\mathrm{LOD}$ & $\mathrm{N} / \mathrm{D}$ & $<\mathrm{LOD}$ & 0.08 & N/D & 0.06 & N/D & N/D & 0.09 & N/D & 1.86 \\
\hline & & $1.5-3.0$ & 0.05 & 0.06 & N/D & 0.15 & 0.16 & N/D & $<\mathrm{LOQ}$ & N/D & N/D & 0.08 & N/D & N/D & N/D & N/D & 1.38 \\
\hline & & $0.95-1.5$ & 0.19 & 0.08 & N/D & $<\mathrm{LOQ}$ & $<\mathrm{LOD}$ & N/D & $<\mathrm{LOD}$ & N/D & N/D & 0.09 & N/D & N/D & N/D & N/D & 1.85 \\
\hline & & $0.49-0.95$ & 0.18 & 0.06 & N/D & $<\mathrm{LOQ}$ & $<\mathrm{LOD}$ & $\mathrm{N} / \mathrm{D}$ & 0.10 & N/D & N/D & 0.87 & N/D & N/D & N/D & N/D & 2.15 \\
\hline & & $<0.49$ & 0.37 & 0.07 & 0.05 & $<\mathrm{LOQ}$ & $<\mathrm{LOD}$ & N/D & 0.07 & N/D & N/D & 0.09 & N/D & N/D & 0.15 & 0.03 & 2.2 \\
\hline & \multirow{6}{*}{$\begin{array}{l}\text { 品 } \\
\text { की }\end{array}$} & $7.2-10$ & 0.09 & 0.03 & 3.79 & $<\mathrm{LOQ}$ & 0.79 & $\mathrm{~N} / \mathrm{D}$ & $<\mathrm{LOD}$ & 0.41 & 0.53 & 0.04 & 1.13 & N/D & N/D & 1.23 & 31.73 \\
\hline & & $3.0-7.2$ & 0.36 & 0.03 & 6.52 & $<\mathrm{LOQ}$ & 1.96 & N/D & $<\mathrm{LOD}$ & 0.92 & 0.98 & 0.07 & 1.93 & N/D & 0.07 & 2.70 & 53.87 \\
\hline & & $1.5-3.0$ & 0.72 & 0.03 & 4.71 & $<\mathrm{LOQ}$ & $<\mathrm{LOD}$ & $\mathrm{N} / \mathrm{D}$ & $<\mathrm{LOD}$ & 0.36 & 0.22 & 0.41 & 2.27 & N/D & 0.07 & 2.06 & 45.53 \\
\hline & & $0.95-1.5$ & 1.88 & 0.04 & 1.96 & $<\mathrm{LOQ}$ & $<\mathrm{LOD}$ & N/D & $<\mathrm{LOD}$ & 0.19 & 0.21 & 0.33 & 1.46 & N/D & 0.09 & 0.93 & 26.02 \\
\hline & & $0.49-0.95$ & 7.47 & 0.08 & 0.54 & $<\mathrm{LOQ}$ & N/D & N/D & $<\mathrm{LOD}$ & 0.14 & 0.31 & 0.06 & 2.33 & N/D & N/D & 0.49 & 39.54 \\
\hline & & $<0.49$ & 36.05 & 0.06 & 2.45 & $<\mathrm{LOQ}$ & $<\mathrm{LOD}$ & N/D & N/D & 0.15 & N/D & 0.61 & 22.05 & N/D & 0.12 & 1.26 & 212.0 \\
\hline & \multirow{6}{*}{ 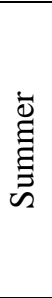 } & $7.2-10$ & 1.00 & 0.04 & N/D & $<\mathrm{LOQ}$ & $<\mathrm{LOD}$ & 0.32 & $<\mathrm{LOD}$ & 1.84 & 0.30 & 0.04 & 0.87 & N/D & N/D & 1.18 & 11.3 \\
\hline & & $3.0-7.2$ & 2.30 & 0.04 & 0.03 & $<\mathrm{LOQ}$ & 0.23 & 0.63 & $<\mathrm{LOD}$ & 3.93 & 0.53 & 0.25 & 1.24 & N/D & 0.08 & 2.08 & 19.14 \\
\hline & & $1.5-3.0$ & 5.06 & 0.04 & N/D & $<\mathrm{LOQ}$ & 0.13 & 0.18 & $<\mathrm{LOD}$ & 1.46 & 0.22 & 0.05 & 1.83 & N/D & 0.08 & 1.69 & 19.86 \\
\hline & & $0.95-1.5$ & 20.98 & 0.04 & 0.04 & $<\mathrm{LOQ}$ & $<\mathrm{LOD}$ & 0.07 & $<\mathrm{LOD}$ & 0.48 & 0.11 & 0.04 & 1.68 & N/D & 0.08 & 1.08 & 33.42 \\
\hline & & 0.49-0.95 & 58.37 & 0.06 & N/D & $<\mathrm{LOQ}$ & N/D & 0.06 & $<\mathrm{LOD}$ & 0.22 & 0.16 & 0.05 & 0.78 & N/D & 0.08 & 0.41 & 66.43 \\
\hline & & $<0.49$ & 110.9 & 0.05 & 0.11 & $<\mathrm{LOQ}$ & N/D & 1.61 & $<\mathrm{LOQ}$ & 0.32 & N/D & 0.06 & 0.84 & N/D & 0.10 & 0.36 & 134.0 \\
\hline & \multirow{6}{*}{$\begin{array}{l}\text { 忢 } \\
\text { 至 }\end{array}$} & $7.2-10$ & 0.27 & $<\mathrm{LOD}$ & 0.65 & $\mathrm{~N} / \mathrm{D}$ & N/D & 1.56 & N/D & 0.11 & N/D & 0.22 & N/D & N/D & $\mathrm{N} / \mathrm{D}$ & $<\mathrm{LOD}$ & 4.18 \\
\hline & & $3.0-7.2$ & 0.05 & N/D & 0.33 & N/D & $\mathrm{N} / \mathrm{D}$ & 0.59 & N/D & 0.13 & N/D & 0.15 & N/D & $\mathrm{N} / \mathrm{D}$ & N/D & $<\mathrm{LOD}$ & 2.41 \\
\hline & & $1.5-3.0$ & 0.22 & N/D & 1.81 & N/D & N/D & 2.61 & N/D & 0.17 & N/D & N/D & $<\mathrm{LOQ}$ & N/D & 0.05 & 0.02 & 8.74 \\
\hline & & $0.95-1.5$ & 0.23 & $<\mathrm{LOQ}$ & 0.25 & N/D & N/D & 1.46 & N/D & N/D & N/D & 0.45 & N/D & N/D & N/D & N/D & 4.57 \\
\hline & & 0.49-0.95 & 0.53 & N/D & 0.05 & N/D & N/D & 1.35 & N/D & N/D & N/D & 0.49 & N/D & 0.05 & 0.08 & N/D & 5.64 \\
\hline & & $<0.49$ & 0.65 & 0.15 & 0.34 & N/D & N/D & 11.44 & N/D & N/D & N/D & 0.87 & N/D & N/D & 0.17 & $<\mathrm{LOQ}$ & 23.34 \\
\hline
\end{tabular}




\begin{tabular}{|c|c|c|c|c|c|c|c|c|c|c|c|c|c|c|c|c|c|}
\hline \multirow{24}{*}{ 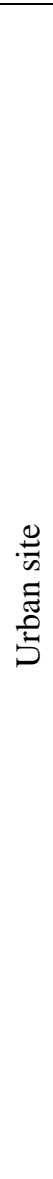 } & \multirow{6}{*}{ 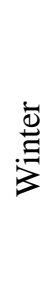 } & $7.2-10$ & 0.04 & N/D & 0.04 & 0.10 & N/D & N/D & N/D & 0.05 & N/D & 0.25 & N/D & 0.01 & N/D & N/D & 2.24 \\
\hline & & $3.0-7.2$ & 0.02 & 0.10 & 0.11 & N/D & N/D & N/D & N/D & N/D & N/D & 0.36 & N/D & N/D & N/D & N/D & 1 \\
\hline & & $1.5-3.0$ & 0.19 & N/D & N/D & $<\mathrm{LOD}$ & N/D & N/D & N/D & N/D & N/D & 0.95 & N/D & 0.02 & 0.03 & N/D & 1.72 \\
\hline & & $0.95-1.5$ & 0.09 & N/D & $<\mathrm{LOD}$ & N/D & N/D & N/D & N/D & N/D & N/D & 0.31 & N/D & N/D & N/D & N/D & 0.98 \\
\hline & & $0.49-0.95$ & 0.20 & 0.39 & $<\mathrm{LOD}$ & N/D & N/D & N/D & 0.43 & 0.04 & N/D & 0.80 & N/D & N/D & N/D & N/D & 8.91 \\
\hline & & $<0.49$ & 0.38 & N/D & 0.04 & $<\mathrm{LOQ}$ & N/D & 1.05 & N/D & 0.11 & N/D & 0.83 & N/D & 0.04 & 0.12 & 0.12 & 15.44 \\
\hline & \multirow{6}{*}{ 告 } & $7.2-10$ & 0.16 & N/D & 4.84 & N/D & N/D & N/D & N/D & 0.20 & 0.65 & 0.28 & 0.57 & N/D & N/D & 1.74 & 13.17 \\
\hline & & $3.0-7.2$ & 0.22 & N/D & 6.26 & N/D & 0.23 & N/D & N/D & 0.22 & 0.87 & 0.30 & 0.49 & 0.03 & N/D & 2.09 & 14.89 \\
\hline & & $1.5-3.0$ & 0.40 & N/D & 2.39 & 0.07 & N/D & N/D & N/D & 0.19 & N/D & 0.17 & 1.30 & N/D & N/D & 1.35 & 11.95 \\
\hline & & $0.95-1.5$ & 1.67 & N/D & 0.95 & N/D & N/D & N/D & N/D & 0.15 & N/D & 0.29 & 1.01 & N/D & N/D & 0.59 & 8.12 \\
\hline & & $0.49-0.95$ & 3.63 & N/D & 0.28 & N/D & N/D & N/D & N/D & 0.09 & N/D & 0.49 & 0.21 & $<\mathrm{LOQ}$ & N/D & 0.09 & 6.32 \\
\hline & & $<0.49$ & 6.59 & N/D & 1.79 & N/D & N/D & 0.03 & N/D & N/D & N/D & 0.85 & 2.31 & N/D & N/D & 0.38 & 21.1 \\
\hline & \multirow{6}{*}{ 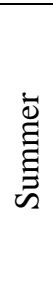 } & $7.2-10$ & 0.24 & 0.08 & N/D & N/D & N/D & 2.48 & N/D & 0.99 & 0.43 & 0.33 & 0.53 & 0.03 & N/D & 0.39 & 9.13 \\
\hline & & $3.0-7.2$ & 0.43 & N/D & N/D & N/D & N/D & 0.97 & N/D & 1.65 & 0.17 & 0.38 & 0.66 & N/D & $<\mathrm{LOQ}$ & 0.54 & 7.97 \\
\hline & & $1.5-3.0$ & 1.67 & N/D & N/D & N/D & N/D & 2.55 & N/D & 0.73 & N/D & 0.31 & 0.88 & N/D & N/D & 0.93 & 12.39 \\
\hline & & $0.95-1.5$ & 1.91 & N/D & N/D & N/D & N/D & 0.65 & 0.56 & 0.15 & N/D & 0.34 & 0.41 & N/D & $<\mathrm{LOQ}$ & 0.44 & 6.12 \\
\hline & & $0.49-0.95$ & 10.85 & N/D & N/D & N/D & N/D & 0.91 & N/D & 0.18 & 1.70 & 0.38 & 0.64 & N/D & N/D & 0.50 & 20.34 \\
\hline & & $<0.49$ & 22.58 & N/D & 0.11 & N/D & N/D & 8.43 & N/D & 0.16 & N/D & 0.91 & 4.67 & N/D & N/D & 2.02 & 56.44 \\
\hline & \multirow{6}{*}{$\underset{\Xi}{\stackrel{\Xi}{\Xi}}$} & $7.2-10$ & 0.04 & N/D & 1.09 & N/D & N/D & 1.17 & N/D & 0.13 & N/D & 0.24 & $<\mathrm{LOQ}$ & N/D & 0.03 & 0.04 & 4.45 \\
\hline & & $3.0-7.2$ & N/D & N/D & 1.77 & N/D & N/D & 1.39 & N/D & 0.28 & N/D & 0.43 & N/D & N/D & $<\mathrm{LOQ}$ & $<\mathrm{LOQ}$ & 5.52 \\
\hline & & $1.5-3.0$ & 0.02 & N/D & 0.71 & N/D & N/D & 0.69 & N/D & 0.15 & N/D & 0.23 & 0.10 & N/D & $<\mathrm{LOQ}$ & $<\mathrm{LOD}$ & 4.04 \\
\hline & & $0.95-1.5$ & 0.07 & N/D & 0.32 & N/D & N/D & 0.46 & N/D & 0.15 & N/D & 0.42 & $<$ LOQ & N/D & 0.04 & 0.06 & 3.64 \\
\hline & & $0.49-0.95$ & 0.11 & N/D & 0.02 & N/D & N/D & 5.02 & N/D & N/D & N/D & 0.38 & $<$ LOD & N/D & 0.02 & N/D & 6.89 \\
\hline & & $<0.49$ & 0.18 & N/D & 0.37 & N/D & N/D & 2.68 & N/D & N/D & N/D & 1.06 & $<\mathrm{LOD}$ & N/D & 0.05 & 0.03 & 6.48 \\
\hline
\end{tabular}


Table S21: Particle-phase concentrations of individual OCPs (in ng.g(PM) ${ }^{-1}$ ) according to particle size (in $\mu \mathrm{m}$ ) at the rural and the urban sites. N/D indicates compounds that were not detected.

\begin{tabular}{|c|c|c|c|c|c|c|c|c|c|c|c|c|c|}
\hline & & $\begin{array}{c}\text { Size } \\
\text { fraction } \\
(\text { in } \mu \mathrm{m})\end{array}$ & $\alpha-\mathrm{HCH}$ & $\beta-\mathrm{HCH}$ & $\gamma-\mathrm{HCH}$ & $\delta-\mathrm{HCH}$ & $o, p^{\prime}-\mathrm{DDE}$ & $p, p^{\prime}-\mathrm{DDE}$ & $o, p^{\prime}-\mathrm{DDD}$ & $p, p^{\prime}-\mathrm{DDD}$ & $o, p^{\prime}-\mathrm{DDT}$ & $p, p^{\prime}$-DDT & $\Sigma O C P S$ \\
\hline \multirow{24}{*}{ 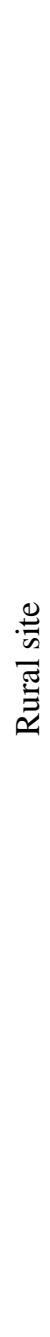 } & \multirow{6}{*}{ 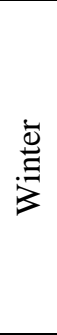 } & 7.2.2010 & 0.39 & 1.31 & 4.68 & 0.98 & 1.33 & 18.24 & 0.92 & 5.30 & 8.38 & 40.09 & 81.6 \\
\hline & & $3.0-7.2$ & 2.20 & 0.24 & 4.48 & 1.06 & 0.72 & 12.38 & 0.76 & 5.03 & 3.40 & 18.76 & 49.0 \\
\hline & & $1.5-3.0$ & 1.96 & 1.45 & 3.62 & 0.38 & 0.82 & 11.02 & 0.67 & 4.66 & 2.65 & 16.39 & 43.6 \\
\hline & & $0.95-1.5$ & 0.38 & 0.39 & 0.97 & 0.40 & 0.69 & 11.18 & 1.10 & 7.16 & 2.00 & 13.59 & 37.9 \\
\hline & & $0.49-0.95$ & N/D & 0.17 & 0.47 & 0.20 & 0.66 & 11.14 & 0.90 & 6.26 & 1.54 & 11.27 & 32.6 \\
\hline & & $<0.49$ & 1.40 & 0.57 & 1.77 & 1.14 & 0.57 & 11.59 & 0.84 & 6.44 & 3.21 & 31.22 & 58.8 \\
\hline & \multirow{6}{*}{ 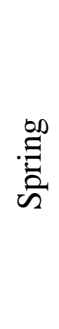 } & 7.2.2010 & 5.77 & 1.11 & 12.72 & 6.27 & 1.34 & 32.54 & 1.00 & 2.74 & 5.19 & 14.00 & 82.7 \\
\hline & & $3.0-7.2$ & 0.94 & N/D & 3.28 & 1.43 & 0.21 & 8.21 & 0.20 & 1.07 & 1.21 & 4.31 & 20.9 \\
\hline & & $1.5-3.0$ & 1.74 & N/D & 4.43 & 1.79 & 0.25 & 10.88 & 0.31 & 1.44 & 1.50 & 6.44 & 28.8 \\
\hline & & $0.95-1.5$ & 2.27 & 0.15 & 4.67 & 1.63 & 0.25 & 11.40 & 0.62 & 1.70 & 2.06 & 11.77 & 36.5 \\
\hline & & $0.49-0.95$ & 0.22 & 0.25 & 1.19 & 0.13 & 0.22 & 11.12 & 0.39 & 2.10 & 1.88 & 11.85 & 29.4 \\
\hline & & $<0.49$ & 0.07 & 2.19 & 0.55 & 0.29 & 0.10 & 3.35 & 0.27 & 0.39 & 1.19 & 8.09 & 16.5 \\
\hline & \multirow{6}{*}{ 㐫 } & 7.2 .2010 & 2.90 & N/D & 15.10 & N/D & 1.59 & 69.95 & 7.63 & 25.04 & 3.58 & N/D & 125.8 \\
\hline & & $3.0-7.2$ & 0.74 & N/D & 2.62 & N/D & 0.30 & 10.38 & 1.33 & 5.14 & 0.57 & 0.88 & 22.0 \\
\hline & & $1.5-3.0$ & 6.48 & 0.36 & 2.34 & N/D & 0.43 & 18.50 & 2.08 & 9.37 & N/D & N/D & 39.6 \\
\hline & & $0.95-1.5$ & 1.22 & N/D & 1.83 & 0.59 & 0.12 & 7.93 & 0.94 & 4.24 & N/D & 0.19 & 17.1 \\
\hline & & $0.49-0.95$ & 1.38 & 0.90 & 1.40 & 0.65 & 0.15 & 6.95 & 0.82 & 3.05 & 0.23 & 1.44 & 17.0 \\
\hline & & $<0.49$ & 0.36 & 1.69 & 0.71 & 0.24 & 0.28 & 9.45 & 0.64 & 3.21 & 0.62 & 1.47 & 18.7 \\
\hline & \multirow{6}{*}{$\underset{\Xi}{\Xi}$} & 7.2.2010 & 0.71 & 2.03 & 2.86 & 0.26 & 1.01 & 43.97 & 4.88 & 17.42 & 0.29 & N/D & 73.4 \\
\hline & & $3.0-7.2$ & 0.10 & N/D & 0.31 & N/D & 0.41 & 19.28 & 1.66 & 8.60 & 0.11 & N/D & 30.5 \\
\hline & & $1.5-3.0$ & N/D & 2.48 & 0.73 & 0.50 & 0.57 & 27.53 & 2.30 & 12.95 & N/D & 0.02 & 47.1 \\
\hline & & $0.95-1.5$ & 0.61 & 0.74 & 0.68 & 0.50 & 0.47 & 24.72 & 1.90 & 12.25 & 0.32 & 0.85 & 43.0 \\
\hline & & $0.49-0.95$ & 0.05 & 0.34 & 0.17 & 0.11 & 0.39 & 24.24 & 1.78 & 12.50 & 0.52 & 1.54 & 41.6 \\
\hline & & $<0.49$ & 0.14 & 0.27 & 0.07 & N/D & 0.29 & 17.57 & 1.36 & 10.15 & 1.00 & 4.00 & 34.9 \\
\hline
\end{tabular}




\begin{tabular}{|c|c|c|c|c|c|c|c|c|c|c|c|c|c|}
\hline \multirow{24}{*}{ 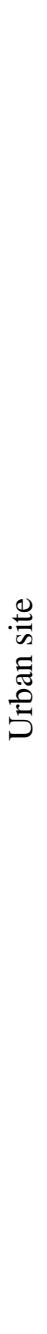 } & \multirow{6}{*}{$\stackrel{\vec{\Xi}}{\stackrel{\Xi}{\Xi}}$} & 7.2.2010 & N/D & 7.27 & 0.93 & N/D & 0.57 & 5.20 & 1.02 & 3.29 & 0.21 & N/D & 18.5 \\
\hline & & $3.0-7.2$ & N/D & 2.86 & 0.40 & N/D & 0.45 & 4.02 & 0.79 & 2.10 & 0.25 & 0.44 & 11.3 \\
\hline & & $1.5-3.0$ & N/D & 1.89 & 0.98 & N/D & 0.95 & 8.99 & 1.53 & 4.62 & 0.50 & 0.85 & 20.3 \\
\hline & & $0.95-1.5$ & N/D & 1.17 & 0.62 & 0.11 & 0.92 & 10.17 & 1.71 & 5.95 & 0.73 & 0.74 & 22.1 \\
\hline & & $0.49-0.95$ & 0.03 & 0.36 & 0.37 & N/D & 0.56 & 5.65 & 1.03 & 4.05 & 0.24 & 0.44 & 12.7 \\
\hline & & $<0.49$ & 0.45 & 0.35 & 0.68 & 0.38 & 0.40 & 4.79 & 1.03 & 3.87 & 0.87 & 0.83 & 13.7 \\
\hline & \multirow{6}{*}{ 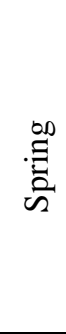 } & 7.2.2010 & 0.16 & 6.03 & 1.00 & 0.38 & 0.55 & 7.42 & 1.76 & 6.27 & 0.65 & 0.61 & 24.8 \\
\hline & & $3.0-7.2$ & 0.63 & 4.54 & 0.93 & 0.34 & 0.40 & 5.60 & 1.21 & 4.31 & 0.38 & 0.44 & 18.8 \\
\hline & & $1.5-3.0$ & N/D & N/D & 0.42 & N/D & 0.62 & 9.36 & 2.15 & 7.69 & 0.50 & 0.39 & 21.1 \\
\hline & & $0.95-1.5$ & N/D & 0.84 & 0.31 & N/D & 0.58 & 8.17 & 1.92 & 6.91 & 0.56 & 0.62 & 19.9 \\
\hline & & $0.49-0.95$ & 0.03 & N/D & 0.45 & N/D & 0.46 & 7.36 & 1.71 & 6.73 & 0.53 & 0.78 & 18.1 \\
\hline & & $<0.49$ & 0.21 & 1.61 & 0.97 & N/D & 0.41 & 6.32 & 1.41 & 5.84 & 0.78 & 1.42 & 19.0 \\
\hline & \multirow{6}{*}{$\begin{array}{l}\bar{\Xi} \\
\Xi \\
\bar{\Xi}\end{array}$} & 7.2.2010 & N/D & 4.63 & 0.38 & $\mathrm{~N} / \mathrm{D}$ & 0.51 & 6.53 & 1.65 & 5.48 & 0.63 & 0.58 & 20.4 \\
\hline & & $3.0-7.2$ & 0.32 & 0.26 & 0.49 & N/D & 0.47 & 6.95 & 1.72 & 6.74 & 0.81 & 1.29 & 19.1 \\
\hline & & $1.5-3.0$ & 2.01 & 2.33 & 1.61 & N/D & 0.74 & 9.70 & 2.55 & 9.41 & 1.63 & 3.50 & 33.5 \\
\hline & & $0.95-1.5$ & 1.60 & 0.94 & 1.29 & N/D & 0.91 & 11.52 & 3.14 & 11.48 & 1.29 & 1.79 & 34.0 \\
\hline & & $0.49-0.95$ & 1.46 & 6.29 & 1.65 & 0.92 & 0.45 & 6.03 & 1.51 & 6.37 & 0.86 & 1.63 & 27.2 \\
\hline & & $<0.49$ & 0.66 & 1.38 & 1.29 & 0.79 & 0.48 & 6.58 & 1.45 & 5.27 & 1.03 & 1.33 & 20.3 \\
\hline & \multirow{6}{*}{$\underset{\Xi}{\Xi}$} & 7.2 .2010 & 0.15 & 3.44 & 1.21 & 0.30 & 0.72 & 14.45 & 1.97 & 8.77 & 1.19 & 2.32 & 34.5 \\
\hline & & $3.0-7.2$ & N/D & 1.78 & 0.26 & N/D & 0.46 & 7.94 & 1.21 & 4.41 & 0.72 & 0.92 & 17.7 \\
\hline & & $1.5-3.0$ & N/D & 2.55 & 0.48 & N/D & 0.75 & 13.00 & 2.34 & 8.89 & 0.84 & 2.00 & 30.9 \\
\hline & & $0.95-1.5$ & $\mathrm{~N} / \mathrm{D}$ & 0.03 & 0.04 & N/D & 0.19 & 3.56 & 0.58 & 2.78 & 0.26 & 0.64 & 8.1 \\
\hline & & $0.49-0.95$ & $\mathrm{~N} / \mathrm{D}$ & 0.07 & 0.17 & N/D & 0.55 & 10.32 & 1.72 & 8.89 & 1.17 & 2.90 & 25.8 \\
\hline & & $<0.49$ & $\mathrm{~N} / \mathrm{D}$ & 0.04 & 0.35 & 0.11 & 0.43 & 9.15 & 1.76 & 7.53 & 1.04 & 2.43 & 22.8 \\
\hline
\end{tabular}


Table S22: Particulate concentrations of individual CUPs (in $\left.\mu \mathrm{g} \cdot \mathrm{g}(\mathrm{PM})^{-1}\right)$ according to particle size (in $\mu \mathrm{m}$ ) at the rural and urban sites. N/D indicates compounds that were not detected, <LOD indicates compounds that were below the limit of detection and $<$ LOQ indicates samples that were below the limit of quantification. LODs and LOQs are found in Table S7.

\begin{tabular}{|c|c|c|c|c|c|c|c|c|c|c|c|c|c|c|c|}
\hline & & 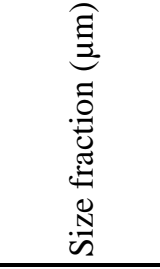 & 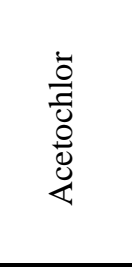 & $\begin{array}{l}\frac{\tilde{0}}{\frac{\pi}{0}} \\
\frac{\tilde{J}}{\gtrless}\end{array}$ & 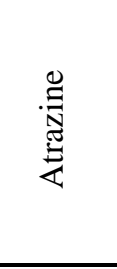 & 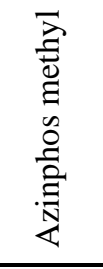 & 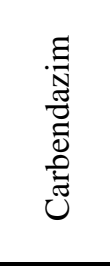 & 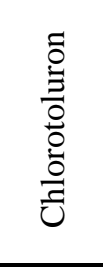 & 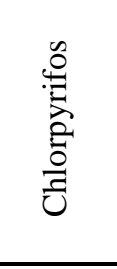 & 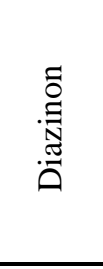 & 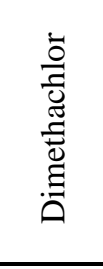 & 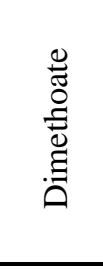 & 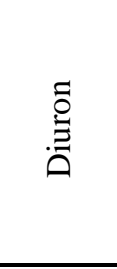 & 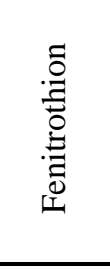 & 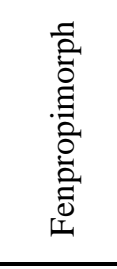 \\
\hline \multirow{22}{*}{ 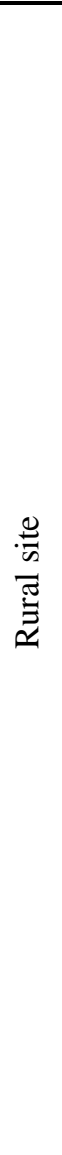 } & \multirow{6}{*}{ 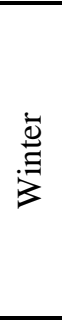 } & $7.2-10$ & 0.097 & $<\mathrm{LOD}$ & 0.376 & N/D & N/D & N/D & $<\mathrm{LOQ}$ & 1.706 & N/D & N/D & $<\mathrm{LOQ}$ & $<\mathrm{LOD}$ & 0.046 \\
\hline & & $3.0-7.2$ & 0.032 & 0.068 & 0.134 & N/D & N/D & N/D & $<\mathrm{LOQ}$ & 0.330 & N/D & N/D & 0.027 & $<\mathrm{LOD}$ & 0.015 \\
\hline & & $1.5-3.0$ & 0.015 & 0.080 & 0.063 & N/D & N/D & N/D & N/D & 0.071 & N/D & N/D & $<\mathrm{LOQ}$ & $<\mathrm{LOD}$ & 0.014 \\
\hline & & $0.95-1.5$ & $<\mathrm{LOQ}$ & $<\mathrm{LOD}$ & 0.096 & N/D & N/D & N/D & $<\mathrm{LOQ}$ & 0.032 & N/D & N/D & 0.021 & $<\mathrm{LOD}$ & 0.019 \\
\hline & & $0.49-0.95$ & 0.007 & $<\mathrm{LOQ}$ & 0.046 & N/D & N/D & N/D & N/D & N/D & N/D & N/D & 0.024 & $<\mathrm{LOD}$ & 0.014 \\
\hline & & $<0.49$ & 0.031 & $<\mathrm{LOD}$ & $\mathrm{N} / \mathrm{D}$ & N/D & $\mathrm{N} / \mathrm{D}$ & N/D & 0.018 & N/D & $\mathrm{N} / \mathrm{D}$ & $\mathrm{N} / \mathrm{D}$ & 0.115 & $<\mathrm{LOD}$ & 0.044 \\
\hline & \multirow{6}{*}{$\begin{array}{l}\stackrel{00}{\Xi} \\
\text { है }\end{array}$} & $7.2-10$ & 33.891 & $<\mathrm{LOQ}$ & $\mathrm{N} / \mathrm{D}$ & 0.086 & 0.717 & 1.156 & 0.430 & 1.937 & N/D & $\mathrm{N} / \mathrm{D}$ & 0.172 & $<\mathrm{LOD}$ & 0.138 \\
\hline & & $3.0-7.2$ & 15.897 & $<\mathrm{LOQ}$ & 0.019 & 0.031 & 0.666 & 0.784 & 0.071 & 0.904 & N/D & N/D & 0.116 & $<\mathrm{LOD}$ & 0.173 \\
\hline & & $1.5-3.0$ & 21.749 & 0.085 & 0.028 & 0.038 & 0.405 & 0.572 & 0.190 & 0.487 & N/D & 0.008 & 0.256 & $<\mathrm{LOD}$ & 0.496 \\
\hline & & $0.95-1.5$ & 6.634 & $<\mathrm{LOD}$ & $\mathrm{N} / \mathrm{D}$ & N/D & 0.072 & N/D & 0.074 & 0.112 & N/D & 0.032 & 0.125 & $<\mathrm{LOD}$ & 0.701 \\
\hline & & $0.49-0.95$ & 4.939 & 0.089 & 0.007 & N/D & N/D & N/D & 0.045 & 0.047 & N/D & 0.049 & 0.098 & $<\mathrm{LOD}$ & 1.401 \\
\hline & & $<0.49$ & 30.076 & $<\mathrm{LOD}$ & 0.071 & N/D & $<\mathrm{LOQ}$ & N/D & 0.139 & 0.199 & N/D & 0.109 & 0.477 & $<\mathrm{LOD}$ & 7.504 \\
\hline & \multirow{6}{*}{ 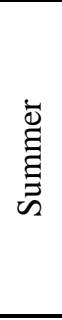 } & $7.2-10$ & 3.225 & 0.373 & 0.077 & $\mathrm{~N} / \mathrm{D}$ & 1.327 & N/D & $<\mathrm{LOQ}$ & 2.546 & N/D & $\mathrm{N} / \mathrm{D}$ & 1.697 & $<\mathrm{LOD}$ & 1.624 \\
\hline & & $3.0-7.2$ & 1.091 & 0.046 & 0.049 & 0.026 & 0.763 & N/D & $<\mathrm{LOQ}$ & 0.299 & N/D & N/D & 0.648 & $<\mathrm{LOD}$ & 0.863 \\
\hline & & $1.5-3.0$ & 3.568 & 0.151 & 0.085 & N/D & 0.728 & $\mathrm{~N} / \mathrm{D}$ & $<\mathrm{LOQ}$ & 1.099 & N/D & N/D & 2.007 & $<\mathrm{LOD}$ & 4.238 \\
\hline & & $0.95-1.5$ & 3.175 & 0.227 & 0.025 & N/D & 0.331 & N/D & $<\mathrm{LOQ}$ & 1.443 & N/D & N/D & 2.089 & $<\mathrm{LOD}$ & 17.329 \\
\hline & & $0.49-0.95$ & 0.427 & 0.101 & N/D & N/D & 0.135 & N/D & $<\mathrm{LOQ}$ & 0.299 & N/D & N/D & 1.154 & $<\mathrm{LOD}$ & 19.805 \\
\hline & & $<0.49$ & 0.191 & 0.138 & N/D & N/D & 0.109 & N/D & 0.020 & 0.051 & N/D & N/D & 2.916 & $<\mathrm{LOD}$ & 19.361 \\
\hline & \multirow{4}{*}{ 当 } & $7.2-10$ & 0.189 & $\mathrm{~N} / \mathrm{D}$ & $<\mathrm{LOQ}$ & N/D & 0.056 & N/D & $<\mathrm{LOQ}$ & 0.327 & N/D & $\mathrm{N} / \mathrm{D}$ & 0.075 & $\mathrm{~N} / \mathrm{D}$ & 0.126 \\
\hline & & $3.0-7.2$ & 0.145 & N/D & $<\mathrm{LOD}$ & N/D & 0.005 & N/D & N/D & 0.188 & N/D & N/D & 0.093 & N/D & 0.017 \\
\hline & & $1.5-3.0$ & 0.220 & $<\mathrm{LOD}$ & N/D & 0.023 & 0.569 & 0.126 & $<\mathrm{LOQ}$ & 0.366 & 0.044 & N/D & 0.140 & N/D & 0.083 \\
\hline & & $0.95-1.5$ & 0.079 & N/D & N/D & N/D & N/D & N/D & $<\mathrm{LOQ}$ & 0.348 & N/D & N/D & 0.227 & $<\mathrm{LOD}$ & 0.070 \\
\hline
\end{tabular}




\begin{tabular}{|c|c|c|c|c|c|c|c|c|c|c|c|c|c|c|c|}
\hline & & $0.49-0.95$ & 0.043 & 0.297 & N/D & N/D & N/D & N/D & $<\mathrm{LOQ}$ & N/D & N/D & N/D & 0.284 & $\mathrm{~N} / \mathrm{D}$ & 0.107 \\
\hline & & $<0.49$ & 0.143 & N/D & N/D & N/D & 0.057 & N/D & $<\mathrm{LOQ}$ & 0.737 & 0.096 & N/D & 1.144 & N/D & 0.145 \\
\hline \multirow{24}{*}{ 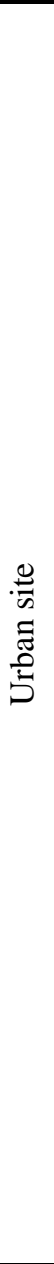 } & \multirow{6}{*}{$\stackrel{\stackrel{\Xi}{\Xi}}{\dot{J}}$} & $7.2-10$ & 0.010 & 0.152 & 0.075 & 0.016 & $\mathrm{~N} / \mathrm{D}$ & $\mathrm{N} / \mathrm{D}$ & $\mathrm{N} / \mathrm{D}$ & 0.276 & $\mathrm{~N} / \mathrm{D}$ & 0.005 & $\mathrm{~N} / \mathrm{D}$ & $\mathrm{N} / \mathrm{D}$ & 0.012 \\
\hline & & $3.0-7.2$ & 0.006 & 0.036 & 0.011 & N/D & $\mathrm{N} / \mathrm{D}$ & N/D & N/D & 0.012 & N/D & N/D & 0.006 & N/D & 0.003 \\
\hline & & $1.5-3.0$ & 0.017 & N/D & 0.106 & $\mathrm{~N} / \mathrm{D}$ & $\mathrm{N} / \mathrm{D}$ & $\mathrm{N} / \mathrm{D}$ & $\mathrm{N} / \mathrm{D}$ & N/D & $\mathrm{N} / \mathrm{D}$ & $\mathrm{N} / \mathrm{D}$ & 0.020 & $\mathrm{~N} / \mathrm{D}$ & 0.050 \\
\hline & & $0.95-1.5$ & 0.010 & $\mathrm{~N} / \mathrm{D}$ & 0.033 & $\mathrm{~N} / \mathrm{D}$ & $\mathrm{N} / \mathrm{D}$ & $\mathrm{N} / \mathrm{D}$ & N/D & 0.028 & N/D & $\mathrm{N} / \mathrm{D}$ & $<\mathrm{LOQ}$ & $\mathrm{N} / \mathrm{D}$ & 0.011 \\
\hline & & $0.49-0.95$ & 0.018 & N/D & 0.049 & N/D & $\mathrm{N} / \mathrm{D}$ & N/D & $<\mathrm{LOQ}$ & N/D & N/D & N/D & 0.005 & 0.545 & 0.018 \\
\hline & & $<0.49$ & 0.031 & 0.841 & 0.083 & N/D & N/D & N/D & 0.053 & N/D & N/D & N/D & 0.016 & N/D & 0.031 \\
\hline & \multirow{6}{*}{ 蒒 } & $7.2-10$ & 0.456 & 0.035 & 0.073 & $\mathrm{~N} / \mathrm{D}$ & 0.149 & 0.133 & $<\mathrm{LOQ}$ & 0.129 & N/D & $\mathrm{N} / \mathrm{D}$ & 0.018 & $\mathrm{~N} / \mathrm{D}$ & 0.035 \\
\hline & & $3.0-7.2$ & 0.221 & 0.035 & 0.005 & N/D & 0.146 & 0.146 & N/D & 0.007 & N/D & N/D & 0.019 & N/D & 0.030 \\
\hline & & $1.5-3.0$ & 1.068 & 0.061 & 0.023 & $\mathrm{~N} / \mathrm{D}$ & 0.181 & 0.023 & 0.032 & 0.344 & N/D & N/D & 0.039 & N/D & 0.115 \\
\hline & & $0.95-1.5$ & 0.630 & N/D & $\mathrm{N} / \mathrm{D}$ & $\mathrm{N} / \mathrm{D}$ & $\mathrm{N} / \mathrm{D}$ & $\mathrm{N} / \mathrm{D}$ & 0.075 & 0.107 & N/D & $\mathrm{N} / \mathrm{D}$ & 0.025 & $\mathrm{~N} / \mathrm{D}$ & 0.403 \\
\hline & & 0.49-0.95 & 0.160 & N/D & 0.008 & N/D & N/D & N/D & 0.023 & 0.000 & N/D & N/D & 0.023 & N/D & 0.511 \\
\hline & & $<0.49$ & 0.628 & N/D & 0.033 & $\mathrm{~N} / \mathrm{D}$ & 0.014 & $\mathrm{~N} / \mathrm{D}$ & 0.027 & 0.000 & N/D & $\mathrm{N} / \mathrm{D}$ & 0.126 & N/D & 0.595 \\
\hline & \multirow{6}{*}{ 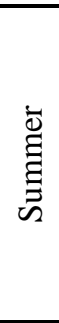 } & $7.2-10$ & 0.291 & $\mathrm{~N} / \mathrm{D}$ & $\mathrm{N} / \mathrm{D}$ & $\mathrm{N} / \mathrm{D}$ & 0.578 & $\mathrm{~N} / \mathrm{D}$ & 0.019 & 0.668 & 0.168 & $\mathrm{~N} / \mathrm{D}$ & 0.030 & $\mathrm{~N} / \mathrm{D}$ & 0.116 \\
\hline & & $3.0-7.2$ & 0.125 & $<\mathrm{LOQ}$ & $<\mathrm{LOQ}$ & $\mathrm{N} / \mathrm{D}$ & 0.278 & $\mathrm{~N} / \mathrm{D}$ & 0.032 & 0.273 & 0.146 & $\mathrm{~N} / \mathrm{D}$ & 0.048 & $\mathrm{~N} / \mathrm{D}$ & 0.123 \\
\hline & & $1.5-3.0$ & 0.790 & N/D & $<\mathrm{LOD}$ & $\mathrm{N} / \mathrm{D}$ & 0.314 & $\mathrm{~N} / \mathrm{D}$ & 0.055 & 0.529 & 0.178 & $\mathrm{~N} / \mathrm{D}$ & 0.521 & $\mathrm{~N} / \mathrm{D}$ & 0.750 \\
\hline & & $0.95-1.5$ & 0.375 & $\mathrm{~N} / \mathrm{D}$ & 0.014 & $\mathrm{~N} / \mathrm{D}$ & 0.119 & $\mathrm{~N} / \mathrm{D}$ & 0.038 & 0.334 & $\mathrm{~N} / \mathrm{D}$ & $\mathrm{N} / \mathrm{D}$ & 0.127 & $\mathrm{~N} / \mathrm{D}$ & 1.159 \\
\hline & & $0.49-0.95$ & 0.181 & $\mathrm{~N} / \mathrm{D}$ & $\mathrm{N} / \mathrm{D}$ & $\mathrm{N} / \mathrm{D}$ & 0.439 & N/D & 0.280 & 0.218 & 0.077 & N/D & 0.176 & N/D & 2.868 \\
\hline & & $<0.49$ & 0.408 & 0.071 & 0.016 & $\mathrm{~N} / \mathrm{D}$ & N/D & $\mathrm{N} / \mathrm{D}$ & 0.036 & 0.248 & 0.848 & N/D & 0.406 & N/D & 2.613 \\
\hline & \multirow{6}{*}{$\underset{\Xi}{\Xi}$} & $7.2-10$ & 0.150 & 0.112 & $\mathrm{~N} / \mathrm{D}$ & $\mathrm{N} / \mathrm{D}$ & 0.028 & $\mathrm{~N} / \mathrm{D}$ & $<\mathrm{LOQ}$ & 0.451 & $\mathrm{~N} / \mathrm{D}$ & $\mathrm{N} / \mathrm{D}$ & 0.022 & $\mathrm{~N} / \mathrm{D}$ & 0.018 \\
\hline & & $3.0-7.2$ & 0.131 & 0.105 & 0.031 & N/D & 0.102 & 0.053 & N/D & N/D & $<\mathrm{LOQ}$ & N/D & 0.043 & N/D & N/D \\
\hline & & $1.5-3.0$ & 0.199 & N/D & N/D & N/D & 0.117 & N/D & $\mathrm{N} / \mathrm{D}$ & 0.806 & 0.058 & N/D & N/D & N/D & 0.010 \\
\hline & & $0.95-1.5$ & 0.105 & 0.231 & $\mathrm{~N} / \mathrm{D}$ & $\mathrm{N} / \mathrm{D}$ & $\mathrm{N} / \mathrm{D}$ & $\mathrm{N} / \mathrm{D}$ & $\mathrm{N} / \mathrm{D}$ & 0.591 & $<\mathrm{LOD}$ & $\mathrm{N} / \mathrm{D}$ & $<\mathrm{LOQ}$ & $<\mathrm{LOD}$ & 0.030 \\
\hline & & $0.49-0.95$ & 0.020 & 0.204 & 0.022 & $\mathrm{~N} / \mathrm{D}$ & 0.003 & $\mathrm{~N} / \mathrm{D}$ & N/D & $\mathrm{N} / \mathrm{D}$ & $\mathrm{N} / \mathrm{D}$ & $\mathrm{N} / \mathrm{D}$ & 0.061 & $\mathrm{~N} / \mathrm{D}$ & 0.026 \\
\hline & & $<0.49$ & 0.028 & 0.079 & 0.014 & N/D & N/D & N/D & $<\mathrm{LOQ}$ & 0.103 & 0.012 & N/D & 0.038 & N/D & 0.023 \\
\hline
\end{tabular}


Table S22 (continued): Particulate concentrations of individual CUPs (in $\mu \mathrm{g} \cdot \mathrm{g}(\mathrm{PM})^{-1}$ ) according to particle size (in $\mu \mathrm{m}$ ) at the rural and the urban sites.

\begin{tabular}{|c|c|c|c|c|c|c|c|c|c|c|c|c|c|c|c|c|}
\hline & & 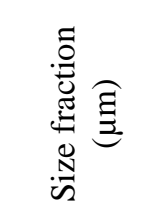 & 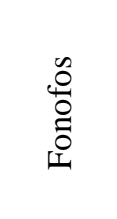 & 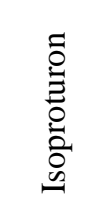 & 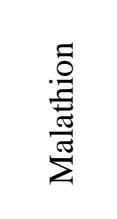 & 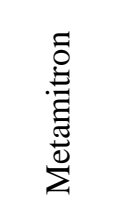 & 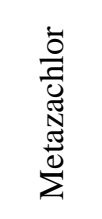 & 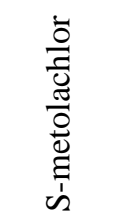 & 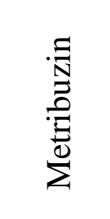 & 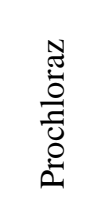 & 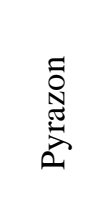 & 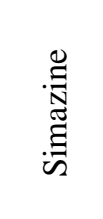 & 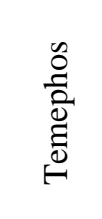 & 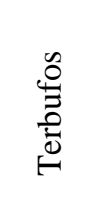 & 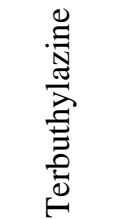 & $\overbrace{\omega}^{\infty}$ \\
\hline \multirow{24}{*}{ 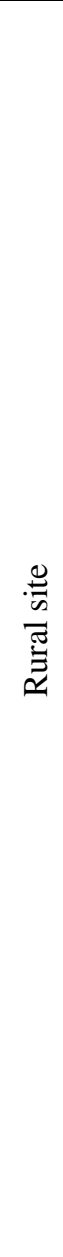 } & \multirow{6}{*}{ 离 } & $7.2-10$ & 0.080 & 0.047 & $<\mathrm{LOQ}$ & $<\mathrm{LOD}$ & $\mathrm{N} / \mathrm{D}$ & $\mathrm{N} / \mathrm{D}$ & $<\mathrm{LOD}$ & $\mathrm{N} / \mathrm{D}$ & N/D & 0.344 & 0.063 & N/D & $\mathrm{N} / \mathrm{D}$ & 2.759 \\
\hline & & $3.0-7.2$ & 0.020 & 0.047 & 0.034 & $<\mathrm{LOD}$ & N/D & N/D & $<\mathrm{LOD}$ & 0.036 & N/D & 0.027 & N/D & 0.038 & N/D & 0.808 \\
\hline & & $1.5-3.0$ & 0.016 & $\mathrm{~N} / \mathrm{D}$ & 0.040 & 0.041 & N/D & N/D & $<\mathrm{LOQ}$ & N/D & N/D & 0.020 & N/D & N/D & N/D & 0.36 \\
\hline & & $0.95-1.5$ & 0.008 & N/D & $<$ LOQ & $<\mathrm{LOD}$ & N/D & N/D & $<\mathrm{LOD}$ & N/D & N/D & 0.009 & N/D & N/D & N/D & 0.185 \\
\hline & & $0.49-0.95$ & 0.005 & N/D & $<\mathrm{LOQ}$ & $<\mathrm{LOD}$ & N/D & N/D & 0.008 & N/D & N/D & 0.071 & N/D & N/D & N/D & 0.175 \\
\hline & & $<0.49$ & 0.009 & 0.006 & $<$ LOQ & $<$ LOD & N/D & N/D & 0.009 & N/D & N/D & 0.011 & N/D & 0.018 & 0.004 & 0.265 \\
\hline & \multirow{6}{*}{ 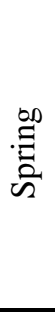 } & $7.2-10$ & 0.055 & 6.142 & $<\mathrm{LOQ}$ & 1.286 & N/D & 1.834 & $<\mathrm{LOD}$ & 0.665 & 0.857 & 0.063 & N/D & $\mathrm{N} / \mathrm{D}$ & 1.991 & 51.42 \\
\hline & & $3.0-7.2$ & 0.016 & 3.142 & $<\mathrm{LOQ}$ & 0.945 & N/D & 0.931 & $<\mathrm{LOD}$ & 0.442 & 0.473 & 0.031 & N/D & 0.032 & 1.303 & 25.976 \\
\hline & & $1.5-3.0$ & 0.023 & 3.237 & $<\mathrm{LOQ}$ & $<\mathrm{LOD}$ & N/D & 1.558 & $<\mathrm{LOD}$ & 0.245 & 0.149 & 0.279 & N/D & 0.046 & 1.414 & 31.265 \\
\hline & & $0.95-1.5$ & 0.017 & 0.729 & $<\mathrm{LOQ}$ & $<\mathrm{LOD}$ & N/D & 0.543 & $<\mathrm{LOD}$ & 0.069 & 0.077 & 0.124 & N/D & 0.034 & 0.345 & 9.688 \\
\hline & & $0.49-0.95$ & 0.015 & 0.101 & $<\mathrm{LOQ}$ & N/D & N/D & 0.436 & $<\mathrm{LOD}$ & 0.026 & 0.058 & 0.011 & N/D & N/D & 0.093 & 7.415 \\
\hline & & $<0.49$ & 0.012 & 0.509 & $<\mathrm{LOQ}$ & $<\mathrm{LOD}$ & N/D & 4.590 & N/D & 0.032 & N/D & 0.127 & N/D & 0.026 & 0.263 & 44.134 \\
\hline & \multirow{6}{*}{ 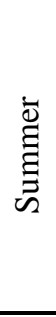 } & $7.2-10$ & 0.061 & $\mathrm{~N} / \mathrm{D}$ & $<\mathrm{LOQ}$ & $<\mathrm{LOD}$ & 0.519 & 1.403 & $<\mathrm{LOD}$ & 2.972 & 0.487 & 0.072 & N/D & $\mathrm{N} / \mathrm{D}$ & 1.915 & 18.298 \\
\hline & & $3.0-7.2$ & 0.015 & 0.012 & $<\mathrm{LOQ}$ & 0.085 & 0.238 & 0.465 & $<\mathrm{LOD}$ & 1.474 & 0.199 & 0.093 & N/D & 0.030 & 0.779 & 7.175 \\
\hline & & $1.5-3.0$ & 0.033 & $\mathrm{~N} / \mathrm{D}$ & $<\mathrm{LOQ}$ & 0.112 & 0.148 & 1.529 & $<\mathrm{LOD}$ & 1.225 & 0.186 & 0.039 & N/D & 0.067 & 1.412 & 16.627 \\
\hline & & $0.95-1.5$ & 0.032 & 0.036 & $<\mathrm{LOQ}$ & $<\mathrm{LOD}$ & 0.058 & 1.386 & $<\mathrm{LOD}$ & 0.400 & 0.089 & 0.037 & N/D & 0.063 & 0.892 & 27.612 \\
\hline & & $0.49-0.95$ & 0.021 & $\mathrm{~N} / \mathrm{D}$ & $<\mathrm{LOQ}$ & N/D & 0.022 & 0.265 & $<\mathrm{LOD}$ & 0.074 & 0.054 & 0.016 & N/D & 0.027 & 0.140 & 22.54 \\
\hline & & $<0.49$ & 0.009 & 0.019 & $<\mathrm{LOQ}$ & N/D & 0.281 & 0.146 & $<\mathrm{LOQ}$ & 0.056 & $\mathrm{~N} / \mathrm{D}$ & 0.010 & N/D & 0.017 & 0.063 & 23.387 \\
\hline & \multirow{6}{*}{$\begin{array}{l}\text { 妾 } \\
\text { 昰 }\end{array}$} & $7.2-10$ & $<\mathrm{LOD}$ & 0.307 & $\mathrm{~N} / \mathrm{D}$ & $\mathrm{N} / \mathrm{D}$ & 0.738 & N/D & N/D & 0.054 & N/D & 0.105 & N/D & N/D & $<\mathrm{LOD}$ & 1.977 \\
\hline & & $3.0-7.2$ & N/D & 0.123 & N/D & N/D & 0.219 & N/D & N/D & 0.047 & N/D & 0.056 & N/D & N/D & $<\mathrm{LOD}$ & 0.893 \\
\hline & & $1.5-3.0$ & $\mathrm{~N} / \mathrm{D}$ & 0.695 & N/D & $\mathrm{N} / \mathrm{D}$ & 1.002 & $<\mathrm{LOQ}$ & N/D & 0.066 & N/D & N/D & N/D & 0.021 & 0.009 & 3.364 \\
\hline & & $0.95-1.5$ & $<\mathrm{LOQ}$ & 0.074 & N/D & $\mathrm{N} / \mathrm{D}$ & 0.436 & N/D & N/D & N/D & N/D & 0.134 & N/D & N/D & N/D & 1.368 \\
\hline & & $0.49-0.95$ & N/D & 0.010 & N/D & $\mathrm{N} / \mathrm{D}$ & 0.273 & N/D & N/D & $\mathrm{N} / \mathrm{D}$ & N/D & 0.099 & 0.009 & 0.016 & N/D & 1.138 \\
\hline & & $<0.49$ & 0.033 & 0.076 & N/D & N/D & 2.563 & N/D & N/D & N/D & N/D & 0.196 & N/D & 0.038 & $<\mathrm{LOQ}$ & 5.228 \\
\hline
\end{tabular}




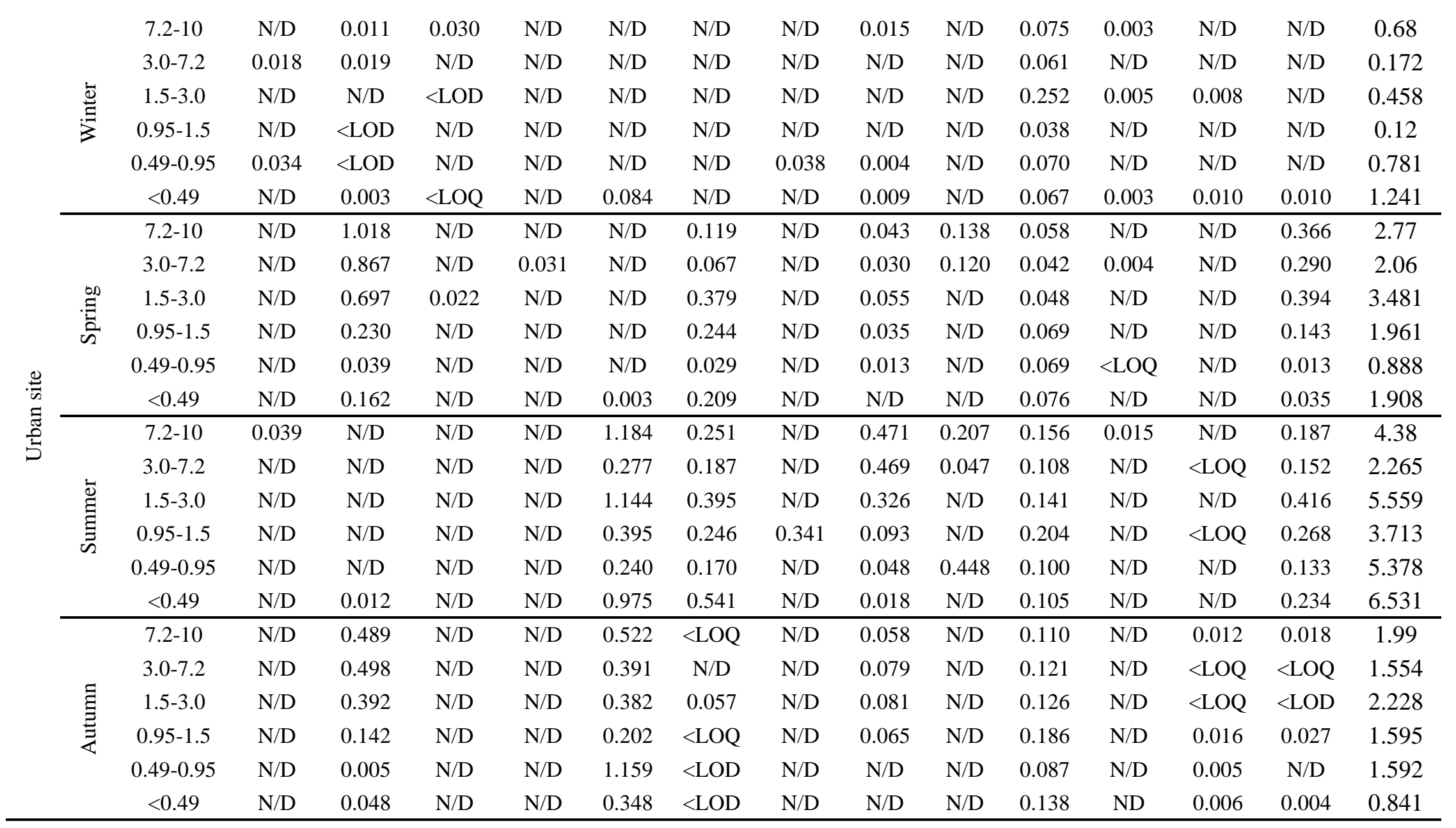




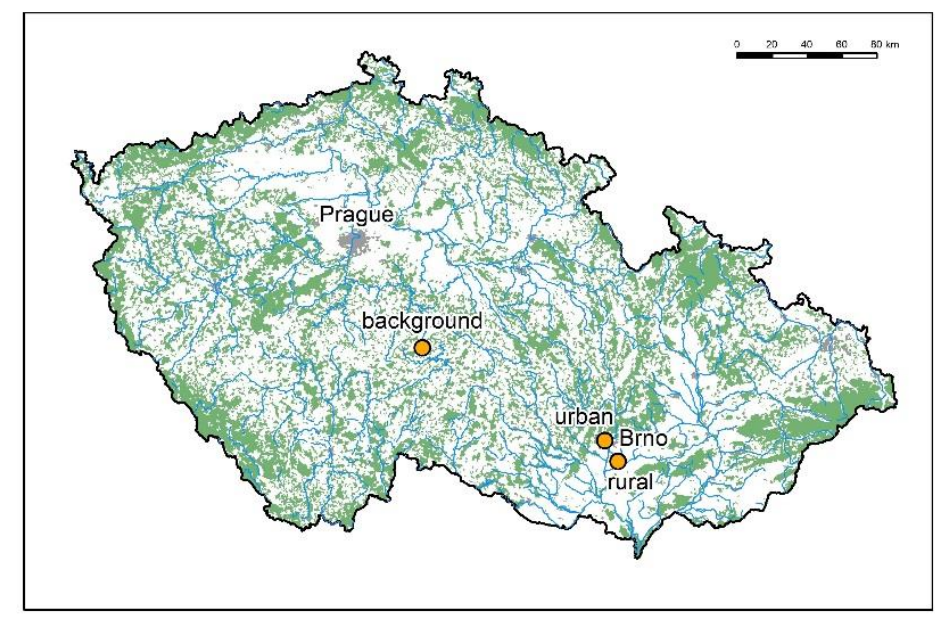

Figure S1: Map of the sampling sites

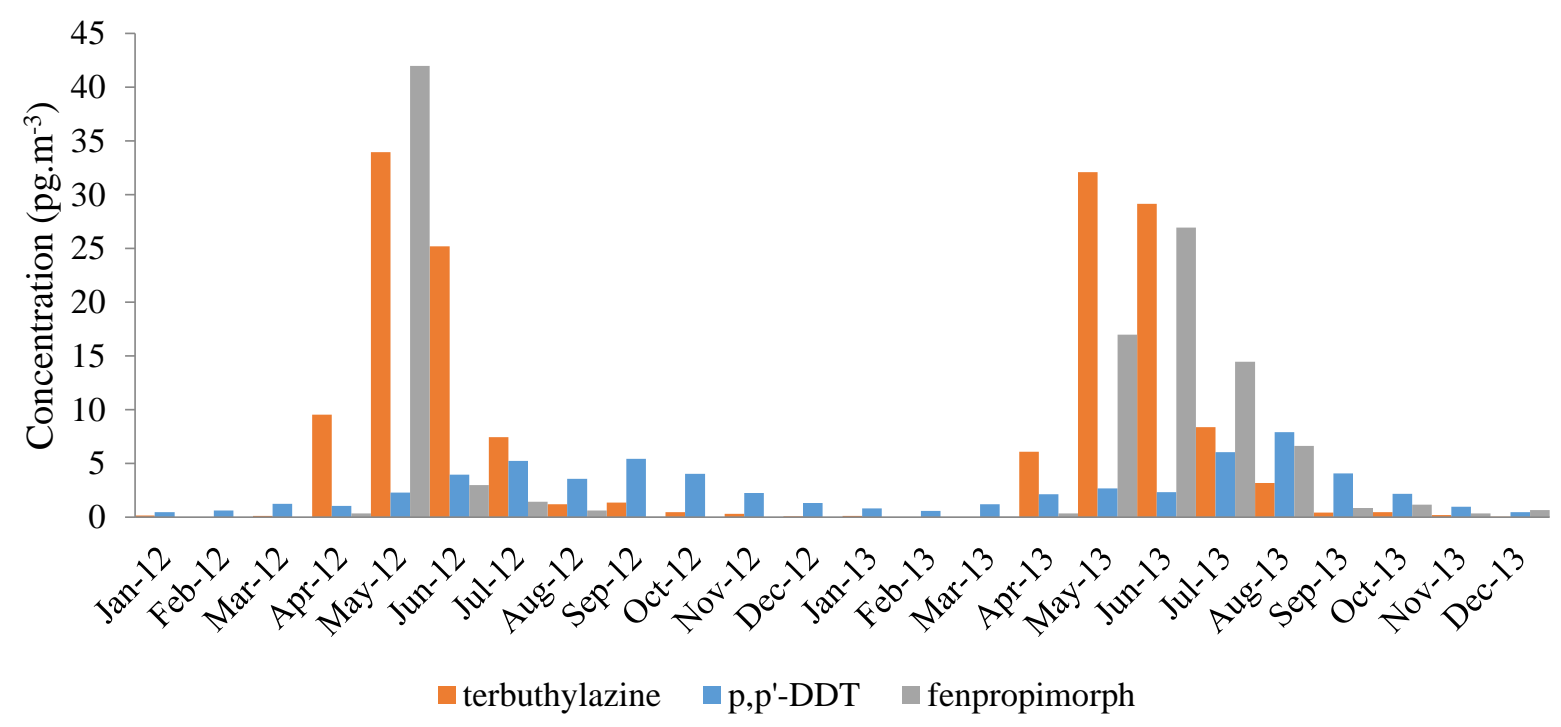

Figure S2: Monthly concentrations (in pg. $\mathrm{m}^{-3}$ ) of terbuthylazine, $p, p^{\prime}$-DDT and fenpropimorph. 

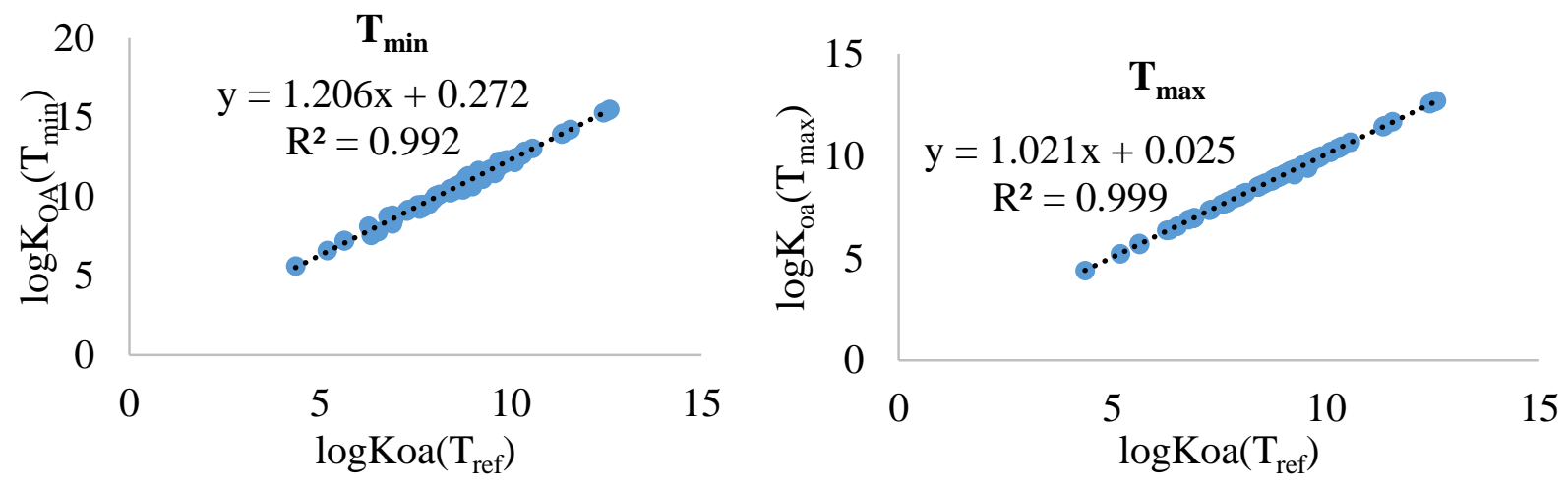

Figure S3: Linear regression of $\log \mathrm{K}_{\mathrm{OA}}(\mathrm{T})$ versus $\log \mathrm{K}_{\mathrm{OA}}\left(\mathrm{T}_{\mathrm{ref}}\right)\left(\mathrm{T}_{\mathrm{ref}}=25^{\circ} \mathrm{C}\right)$ of OCPs, CBs, PCBs, PAHs, CAR and PCNs for $\mathrm{T}_{\min }\left(\mathrm{T}_{\min }=-13.3^{\circ} \mathrm{C}\right)$ and $\mathrm{T}_{\max }\left(\mathrm{T}_{\max }=22.8^{\circ} \mathrm{C}\right)$ used to determine the $\log \mathrm{K}_{\mathrm{OA}}$ of all CUPs except chlorpyrifos. Experimental values from references 7-13.(Harner and Bidleman, 1998a; Harner and Mackay, 1995; Odabasi and Cetin, 2012b; Odabasi et al., 2006a, 2006b; Shoeib and Harner, 2002b; Zhang et al., 2009)

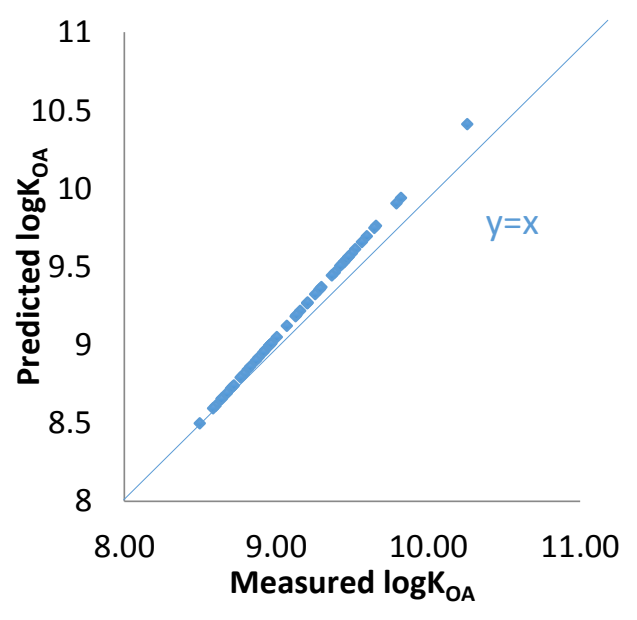

Figure S4: Comparison of $\log \mathrm{K}_{\mathrm{OA}}$ values of chlorpyrifos at different temperatures determined based on experimental values (Odabasi and Cetin, 2012b) and extrapolated from the regression analysis. 


\section{References}

Coscollà, C., Hart, E., Pastor, A. and Yusà, V.: LC-MS characterization of contemporary pesticides in PM10 of Valencia Region, Spain, Atmos. Environ., 77(0), 394-403, doi:http://dx.doi.org/10.1016/j.atmosenv.2013.05.022, 2013a.

Coscollà, C., Yahyaoui, A., Colin, P., Robin, C., Martinon, L., Val, S., Baeza-Squiban, A., Mellouki, A. and Yusà, V.: Particle size distributions of currently used pesticides in a rural atmosphere of France, Atmos. Environ., 81, 32-38, doi:10.1016/j.atmosenv.2013.08.057, 2013b.

Götz, C. W., Scheringer, M., MacLeod, M., Roth, C. M. and Hungerbühler, K.: Alternative Approaches for Modeling Gas-Particle Partitioning of Semivolatile Organic Chemicals: Model Development and Comparison, Environ. Sci. Technol., 41(4), 1272-1278, doi:10.1021/es060583y, 2007.

Harner, T. and Bidleman, T. F.: Measurement of octanol-air partition coefficients for polycyclic aromatic hydrocarbons and polychlorinated naphthalenes, J. Chem. Eng. Data, 9568(2), 40-46, 1998a.

Harner, T. and Bidleman, T. F.: Octanol-Air Partition Coefficient for Describing Particle/Gas Partitioning of Aromatic Compounds in Urban Air, Environ. Sci. Technol., 32(10), 1494-1502, doi:10.1021/es970890r, 1998b.

Harner, T. and Mackay, D.: Measurement of Octanol-Air Partition Coefficients for Chlorobenzenes, PCBs, and DDT, Environ. Sci. Technol., 29(6), 1599-1606, doi:10.1021/es00006a025, 1995.

Odabasi, M. and Cetin, B.: Determination of octanol-air partition coefficients of organochlorine pesticides (OCPs) as a function of temperature: Application to air-soil exchange, J. Environ. Manage., 113, 432439, doi:10.1016/j.jenvman.2012.10.010, 2012a.

Odabasi, M. and Cetin, B.: Determination of octanol-air partition coefficients of organochlorine pesticides (OCPs) as a function of temperature: application to air-soil exchange, J. Environ. Manage., 113, 432-9, 2012b.

Odabasi, M., Cetin, E. and Sofuoglu, A.: Determination of octanol-air partition coefficients and supercooled liquid vapor pressures of PAHs as a function of temperature: Application to gas-particle partitioning in an urban atmosphere, Atmos. Environ., 40(34), 6615-6625, doi:http://dx.doi.org/10.1016/j.atmosenv.2006.05.051, 2006a.

Odabasi, M., Cetin, B. and Sofuoglu, A.: Henry's law constant, octanol-air partition coefficient and supercooled liquid vapor pressure of carbazole as a function of temperature: Application to gas/particle partitioning in the atmosphere, Chemosphere, 62(7), 1087-1096, doi:http://dx.doi.org/10.1016/j.chemosphere.2005.05.035, 2006b.

Putaud, J.-P., Raes, F., Van Dingenen, R., Brüggemann, E., Facchini, M. C., Decesari, S., Fuzzi, S., Gehrig, R., Hüglin, C., Laj, P., Lorbeer, G., Maenhaut, W., Mihalopoulos, N., Müller, K., Querol, X., Rodriguez, S., Schneider, J., Spindler, G., Brink, H. ten, Tørseth, K. and Wiedensohler, A.: A European aerosol phenomenology-2: chemical characteristics of particulate matter at kerbside, urban, rural and background sites in Europe, Atmos. Environ., 38(16), 2579-2595, doi:http://dx.doi.org/10.1016/j.atmosenv.2004.01.041, 2004. 
Sadiki, M. and Poissant, L.: Atmospheric concentrations and gas-particle partitions of pesticides: Comparisons between measured and gas-particle partitioning models from source and receptor sites, Atmos. Environ., 42(35), 8288-8299, doi:http://dx.doi.org/10.1016/j.atmosenv.2008.07.041, 2008.

Sauret, N., Wortham, H., Putaud, J.-P. and Mirabel, P.: Study of the effects of environmental parameters on the gas/particle partitioning of current-use pesticides in urban air, Atmos. Environ., 42(3), 544-553, doi:http://dx.doi.org/10.1016/j.atmosenv.2007.09.012, 2008.

Shoeib, M. and Harner, T.: Using measured octanol-air partition coefficients to explain environmental partitioning of organochlorine pesticides, Environ. Toxicol. Chem., 21(5), 984-990, doi:10.1897/15515028(2002)021<0984:umoapc>2.0.co;2, 2002a.

Shoeib, M. and Harner, T.: Using measured octanol-air partition coefficients to explain environmental partitioning of organochlorine pesticides., Environ. Toxicol. Chem., 21(5), 984-990, doi:10.1897/15515028(2002)021<0984:umoapc>2.0.co;2, 2002b.

SRS: State Phytosanitary Administration. Usage of active substances in 2012, Czech Republic. http://eagri.cz/public/web/file/242789/spotreba_UL_2012_anglicka_verze_.pdf, 2013.

SRS: State Phytosanitary Administration. Usage of active substances in 2013, Czech Republic. http://eagri.cz/public/web/file/317733/Spotreba_UL_2013_angl_verze.pdf, 2014.

U.S. EPA: Estimation Programs Interface Suite (EPI Suite) for Microsoft Windows, v 4.11, 2012.

Yao, Y., Harner, T., Blanchard, P., Tuduri, L., Waite, D., Poissant, L., Murphy, C., Belzer, W., Aulagnier, F. and Sverko, E.: Pesticides in the Atmosphere Across Canadian Agricultural Regions, Environ. Sci. Technol., 42(16), 5931-5937, doi:10.1021/es800878r, 2008.

Zhang, N., Yang, Y., Liu, Y. and Tao, S.: Determination of octanol-air partition coefficients and supercooled liquid vapor pressures of organochlorine pesticides, J. Environ. Sci. Heal. Part B, 44(7), 649656, 2009. 